\title{
INFORMACIÓN, GESTIÓN Y ECONOMÍA Reflexiones desde la experiencia ecuatoriana
}

\section{FOREX v Autores:}

Edinson Palacios-Trujillo | Susana Naranjo Ordóñez | Javier Palacios Trujillo | Armida de Jesús Fernández | Francisca Cazorla Logroño Wilman G. Carrillo-Pulgar I María E. Borja-Lombeida | Daysi Astudillo Condo I Fausto Erazo Guijarro | Sandra Huilcapi Peñafiel Pablo Ochoa-Ulloa | Eduardo G. Zurita-Moreano | María G. González-Bautista

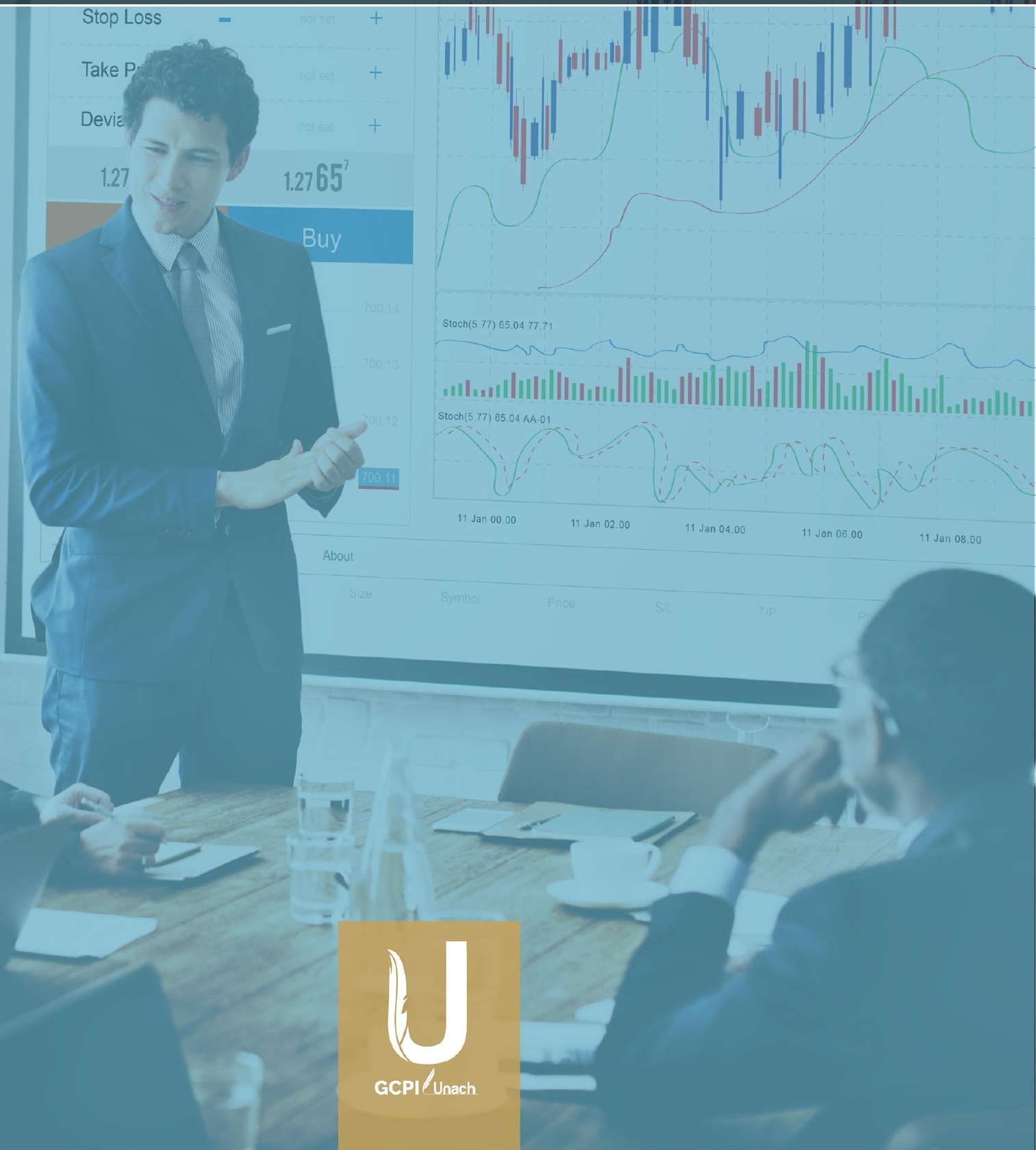





\section{INFORMACIÓN, GESTIÓN Y ECONOMÍA Reflexiones desde la experiencia ecuatoriana}

\section{Editor:}

\section{Stop Loss _ - Diego E. Pinilla-Rodríguez}

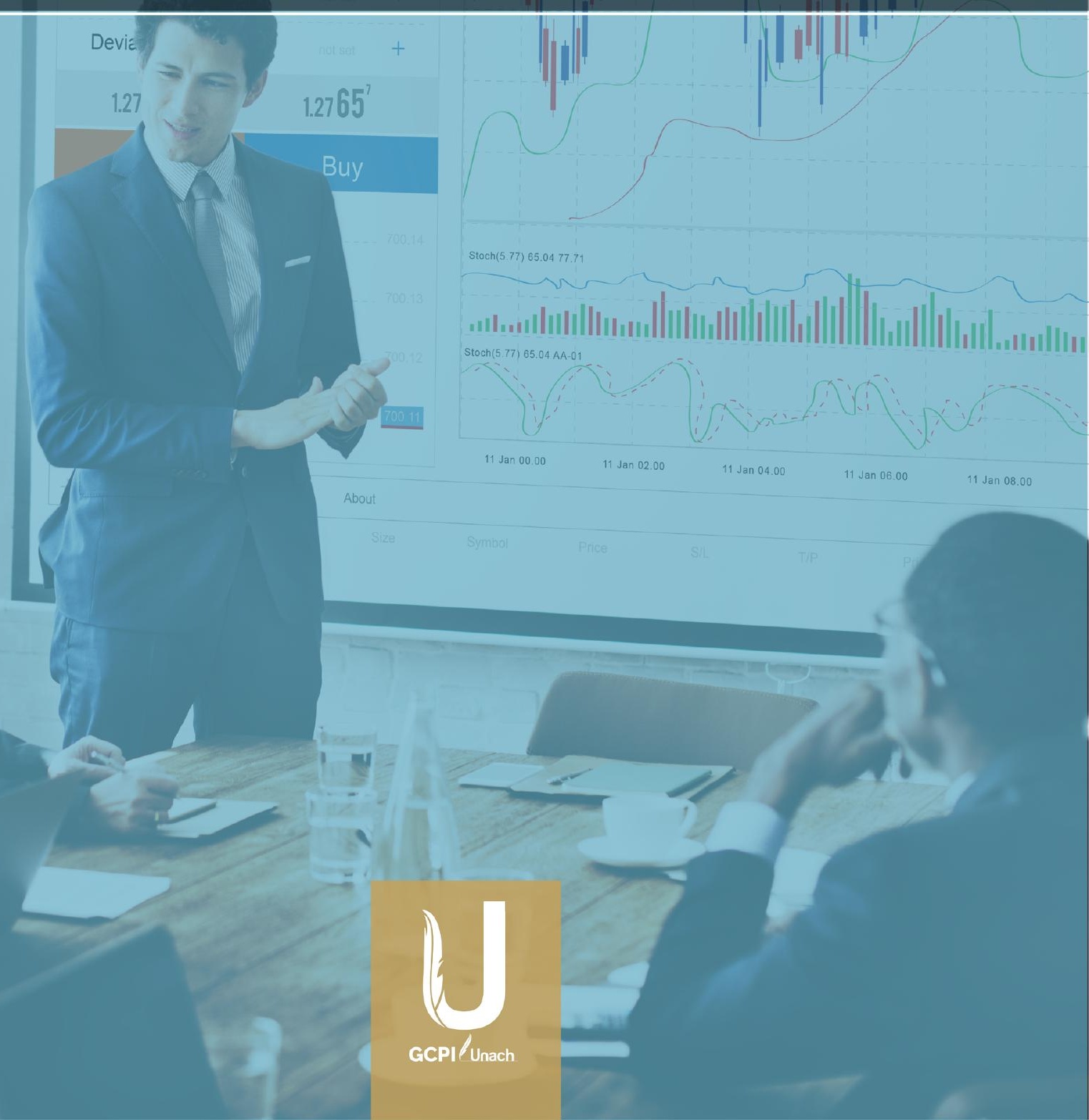




\section{UNIVERSIDAD NACIONAL DE CHIMBORAZO}

Rector

Ph.D. Gonzalo Nicolay Samaniego Erazo

Vicerrectora Académica

Ph.D. Lida Mercedes Barba Maggi

Vicerrector de Investigación, Vinculación y Posgrado

Ph.D. Luis Alberto Tuaza Castro

Vicerrectora Administrativa

Mag. Yolanda Elizabeth Salazar Granizo

Comité Editorial:

Presidente: Ph.D. Luis Alberto Tuaza Castro

Secretaria: Mag. Sandra Zúñiga Donoso

Miembros: Ph.D. Anita Ríos Rivera; Ph.D. Víctor Julio García; Ph.D. Gerardo Nieves Loja; Ph.D. Carmen Varguillas Carmona; Ph.D. Cristhy Jiménez Granizo; Ph.D. Pablo Djabayan Djibeyan; Ph.D. Magda Cejas Martínez; Ph.D. Cristian Naranjo Navas

Título de la obra: INFORMACIÓN, GESTIÓN Y ECONOMÍA Reflexiones desde la experiencia ecuatoriana

Nombres de los autores: Edinson Palacios-Trujillo; Susana Naranjo Ordóñez; Javier Palacios Trujillo; Armida de Jesús Fernández; Francisca Cazorla Logroño Wilman G. Carrillo-Pulgar; María E. Borja-Lombeida; Daysi Astudillo Condo; Fausto Erazo Guijarro; Sandra Huilcapi Peñafiel; Pablo Ochoa-Ulloa I Eduardo G. Zurita-Moreano; María G. González-Bautista; Riobamba, 2021

(C) UNACH, 2021

Ediciones: Universidad Nacional de Chimborazo (UNACH)

Diseño Gráfico: UNACH

Primera edición - enero 2022

Riobamba - Ecuador

Derechos reservados. Se prohíbe la reproducción de esta obra por cualquier medio impreso, reprográfico o electrónico. El contenido, uso de fotografías, gráficos, cuadros, tablas, y referencias es de exclusiva responsabilidad de los autores

ISBN: 978-9942-835-78-9

ISBN: 978-9942-835-79-6 (DIGITAL)

Registro Biblioteca Nacional

Depósito legal: 061173

DOI: https: / / doi.org/10.37135/u.editorial.05.50

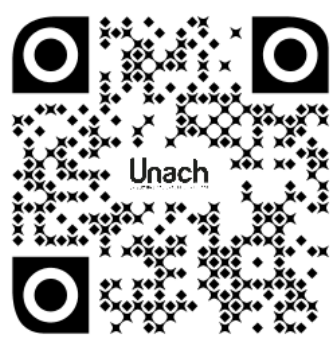




\section{INFORMACIÓN, GESTIÓN Y ECONOMÍA Reflexiones desde la experiencia ecuatoriana}

Filiación autores:

Edinson Palacios-Trujillo

Universidad Nacional de Chimborazo eppalacios@unach.edu.ec

Susana Naranjo Ordóñez

Escuela Superior Politécnica de Chimborazo laura.naranjo@espoch.edu.ec

Javier Palacios Trujillo Investigador independiente jjavierpalacios@hotmail.com

Armida de Jesús Fernández Universidad Central de Venezuela armida.fernandez@ucv.ve

Francisca Cazorla Logroño Universidad Nacional de Chimborazo francisca.cazorla@unach.edu.ec

Wilman G. Carrillo-Pulgar Universidad Nacional de Chimborazo wcarrillo@unach.edu.ec

María E. Borja-Lombeida Universidad Nacional de Chimborazo mborja@unach.edu.ec
Daysi Astudillo Condo

Universidad Nacional de Chimborazo dastudillo@unach.edu.ec

Fausto Erazo Guijarro

Universidad Nacional de Chimborazo fausto.erazo@unach.edu.ec

Sandra Huilcapi Peñafiel

Universidad Nacional de Chimborazo shuilcapi@unach.edu.ec

Pablo Ochoa-Ulloa

Universidad Nacional de Chimborazo pablo.ochoa@unach.edu.ec

Eduardo G. Zurita-Moreano Universidad Nacional de Chimborazo ezurita@unach.edu.ec

María G. González-Bautista Universidad Nacional de Chimborazo mggonzalez@unach.edu.ec 


\section{ÍNDICE}

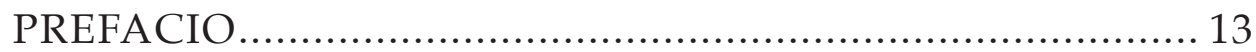

CAPÍTULO 1.

TOMANDO DECISIONES GERENCIALES CON EL APOYO

DE LAS TECNOLOGÍAS DE LA INFORMACIÓN............... 24

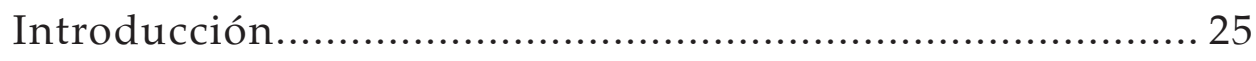

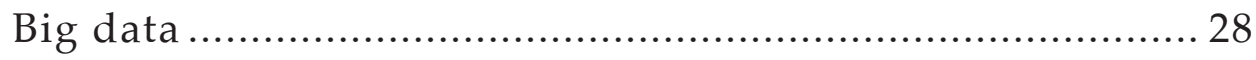

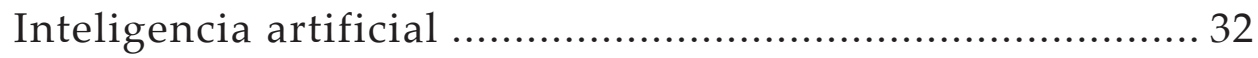

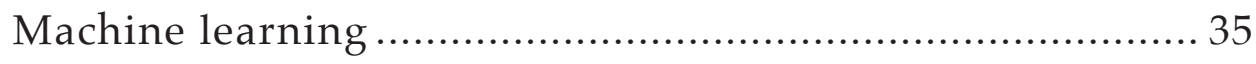

Business intelligence …............................................ 39

Sistema de información para toma de decisiones................ 41

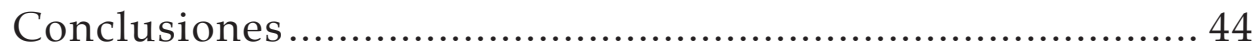

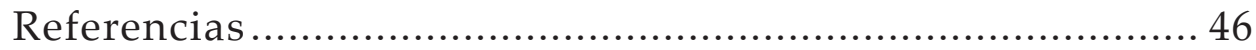

CAPÍTULO 2.

ENCUESTA DE OPINIÓN PARA LA EVALUACIÓN DE LA GESTIÓN PÚBLICA EN LA CIUDAD DE RIOBAMBA AL

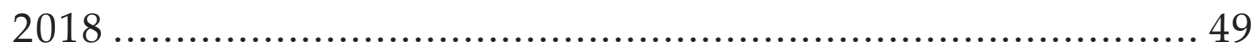

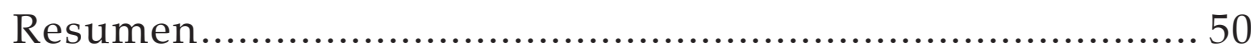

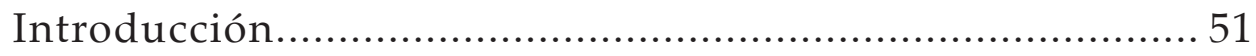

¿Qué es la opinión pública? Presión social y racionalidad . 52 Algunos problemas de la opinión pública 54

La encuesta de opinión pública como instrumento de investigación. 55

Importancia, límites y excesos de las encuestas de opinión 
pública 57

Imparcialidad ligada a la encuesta de opinión pública....... 59

Métodos cualitativos y cuantitativos ................................. 60

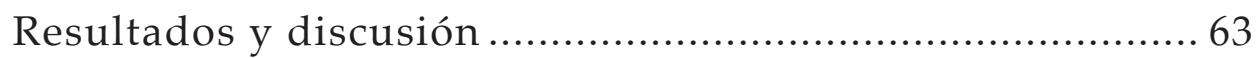

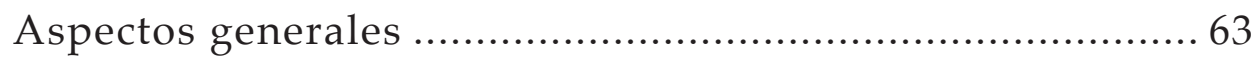

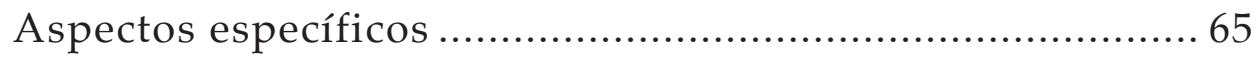

Imagen que tienen los riobambeños de las instituciones de la ciudad 65

Nivel de credibilidad de las autoridades de la ciudad de Riobamba 66

Opinión sobre la calidad de los servicios .......................... 67

Instituciones de educación superio .............................. 68

Instituciones productivas más representativas................... 69

Aspectos locales más relevantes.................................... 70

Intención de voto de los posibles candidatos para la alcaldía

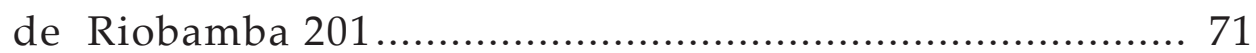

Seguridad ciudadana .................................................. 72

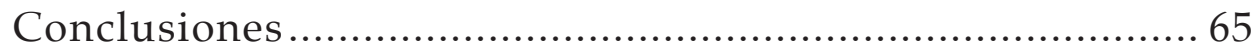

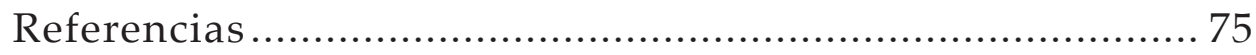

CAPÍTULO 3.

THE ECUADORIAN FLOWER CLUSTER......................... 77

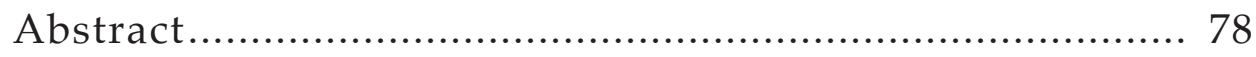

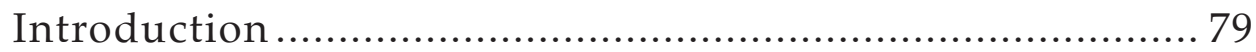

The ecuadorian world cluster status ............................... 80

Relevant cluster charts: flower industry status in Ecuador 81

Education in Ecuador 82 
Ecuador: evolution of flowers exporting ......................... 84

Ecuador: total exports by type of flower ......................... 85

Ecuador: market share ................................................ 85

Prices by type of flowers ........................................... 87

Characteristics of the equatorian rose.............................. 88

Geographical location of rose crops.............................. 88

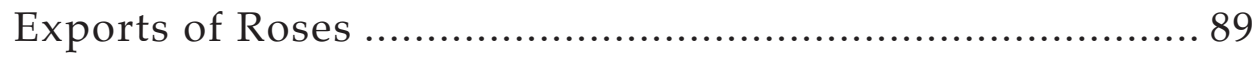

Ecuadorian export destinations ...................................... 90

Competitive advantage of Ecuador as a supplier............... 91

Innovation, research, and development of the sector ........ 92

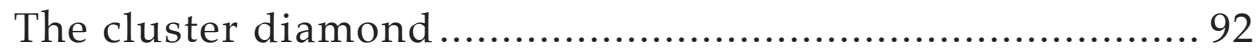

Synthesis of current situation …............................. 107

Recommendations derived from the analysis including three relevant projects, including a shared value project...........113

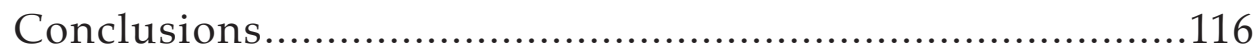

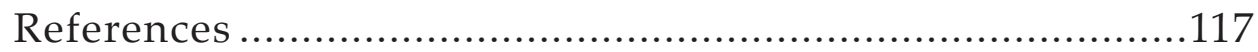

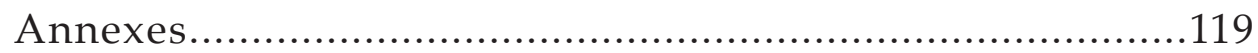

CAPÍTULO 4.

LOS CICLOS ECONÓMICOS. UN ANÁLISIS

MICROECONÓMICO ................................................ 125

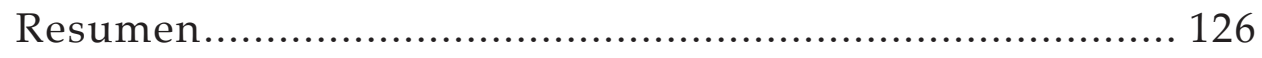

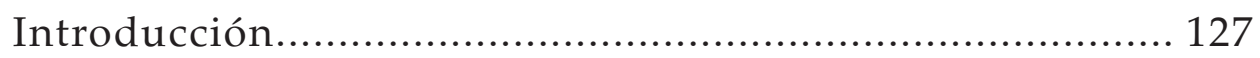

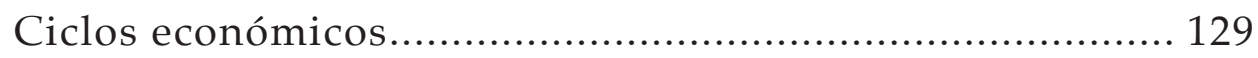

Tipos de ciclos económicos......................................... 135

Teorías de los ciclos económicos ................................ 137

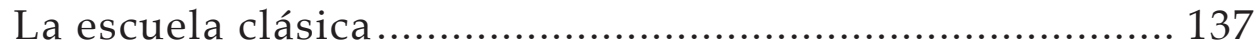


La escuela keynesiana ................................................. 140

El enfoque de Slutsky y Frisch ...................................... 143

Neoclásico keynesiano.................................................. 147

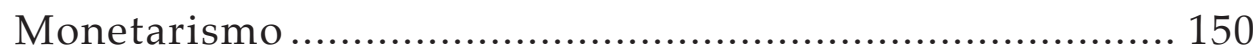

La nueva economía clásica ......................................... 150

Ciclos económicos reales ............................................... 151

Modelo básico del ciclo económico real........................... 152

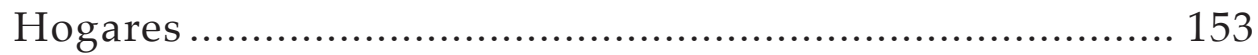

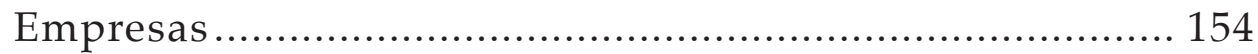

Fundamentos teóricos de los modelos RBC ...................... 156

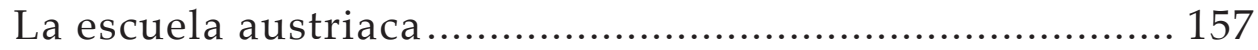

Los ciclos económicos del Ecuador ............................... 158

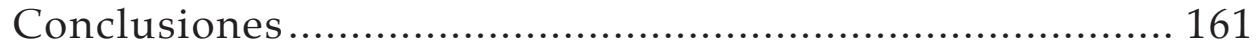


Índice de tablas

Tabla 1. Unidades de medida de datos. ............................ 29

Tabla 2. Beneficios e inconvenientes del big data............... 30

Tabla 3. Algoritmos de machine learning para usos

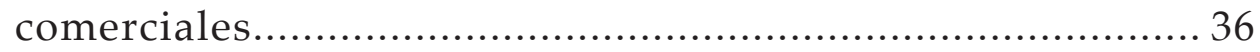

Tabla 4. Algoritmos de deep learning para usos comerciales.................................................. 38

Tabla 5. Ventajas y desventajas de las encuestas de opinión

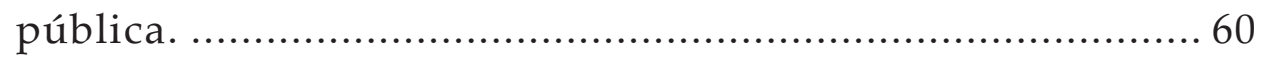

Tabla 6. Número de encuestas por parroquias.................... 61

Tabla 7. Ficha metodológica. ........................................ 61

Tabla 8. Escala tipo Likert, aplicado a la calidad y suministro de servicios públicos y la imagen de instituciones públicas y privadas. 62

Tabla 9. Escala tipo Likert, aplicado a la credibilidad de las autoridades de la provincia. 62

Table 10. Prices by type of flowers.................................. 87

Table 11. Exports of roses.............................................. 90 
Índice de figuras

Figura 1. Cinco enfoques y capacidades para impulsar el modelo operativo de la próxima generación.

Figura 2. Funciones del business intelligence. ................. 40

Figura 3. Aplicaciones del business intelligence................ 41

Figura 4. Distribución por sexo...................................... 64

Figura 5. Edades de las personas encuestadas. .................. 64

Figura 6. Nivel de instrucción de las personas

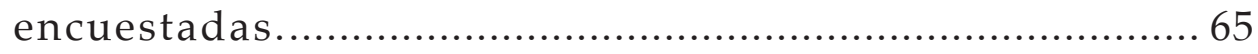

Figura 7. Imagen de las instituciones de la ciudad de

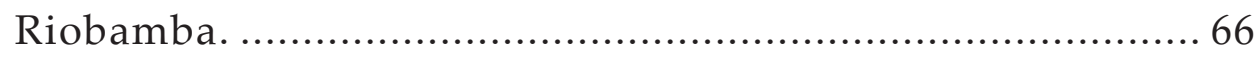

Figura 8. Nivel de credibilidad de las autoridades de Riobamba.

Figura 9. Encuesta sobre la calidad de los servicios públicos.

Figura 10. Imagen de las instituciones de educación superior de Riobamba.

Figura 11. Imagen de las instituciones de producción de Riobamba. 70

Figura 12. Calidad del servicio de transporte en buses y taxis.

Figura 13. Intención de voto de los posibles candidatos para la Alcaldía de Riobamba 2019. 72

Figura 14. Percepción de la seguridad ciudadana en Riobamba. 73

Figure 15. Annual economic growth. 82 
Figure 16. Global competitiveness report - Ecuador. 83

Figure 17. The most problematic factors for doing business.

Figure 18. Total exports of flowers in Ecuador. 85

Figure 19. Market share 2015 - 2016. 86

Figure 20. Geographical location of rose crops. 89

Figure 21. Export destinations. 91

Figure 22. The cluster diamond analysis of roses in Ecuador.

Figure 23. The value chain in the case of the roses of Ecuador. 98

Figure 24. The value chain in the case of the roses of Ecuador.

Figure 25. Five force analysis of the ecuadorian flower industry 105

Figure 26. Process of the cultivation of the roses. 108

Figura 27. Fases de los ciclos económicos..... 132

Figura 28. Tipos de ciclos económicos. 135

Figura 29. Curva de Phillips..... 146

Figura 30. Producto interno bruto de ecuador (precios constantes). Período 1970-2019. 159

Referencias 164 
La información es un elemento fundamental en la economía; ya sea en el ejercicio de lo público o de lo privado, desde lo macro hasta la circunstancia más cotidiana, la información está presente. La información y el acceso a la misma juega un papel cada vez más importante para el funcionamiento de las empresas, el mercado y el gobierno. La información es en sí una fuerza productiva y la base de todas las decisiones. Por tanto, su acceso y gestión es un factor fundamental. Es un derecho y un componente trascendental del desarrollo.

La información es un activo que actualmente se ha convertido en un elemento clave en la estrategia competitiva de países y empresas. En las sociedades actuales, solo perviven las organizaciones que gestionan su información y crean nuevos conocimientos y los gestionan adecuadamente. Es decir, aquellas que reconocen a la información como un recurso indispensable para ampliar su competitividad y aumentar la calidad de los servicios que prestan a ciudadanos y clientes. Las organizaciones serán más valoradas en la medida que tengan una mayor capacidad para gestionar su información, y que esto sea la base para su competitividad y su conexión con clientes y ciudadanos.

Ahora bien, la información que surge de datos u observaciones debe ser interpretada para que surja el conocimiento. Por tanto, es necesaria una capacidad de interpretar. La información son datos dentro de un contexto y el conocimiento es información sujeta a la reflexión y a otras prácticas que provean un entendimiento más profundo (Erickson y Rothberg, 2014). Actualmente, este proceso está profundamente mediado por las tecnologías de la información, elemento indispensable para el manejo eficiente de las cantidades ingentes de datos que se generan. 
Es necesaria la transformación de la información en conocimiento. Es ineludible el desarrollo de procesos que vayan más allá de una mera recolección y transmisión de datos. Bajo este desafío, han surgido todo un conjunto de disciplinas ligadas al desarrollo de nuevas tecnologías y a la gestión de los asuntos públicos y privados, que implementan teorías y procesos con el fin de lograr que un intangible como el conocimiento, genere riqueza y bienestar social. Al respecto, el horizonte que se presenta en términos teóricos como prácticos es amplio. No existe un modelo universal para la gestión del conocimiento, su aplicación e implementación. Se puede afirmar que aún existe un paradigma en construcción, que se puede enriquecer con diversas experiencias y de distintas latitudes.

Este sería el objetivo del presente libro: abordar un conjunto de experiencias puntuales en el ámbito ecuatoriano, que permitan vislumbrar los avances hasta ahora realizados en áreas tan disímiles como la medición y la gestión de la información que surge de la opinión pública, el papel de las tecnologías de la información en la gestión privada, un clúster productivo como gestor de su conocimiento y ventajas competitivas, y la gestión macro de los ciclos económicos, a partir de colectar y procesar información, tanto teórica como empírica. De este modo, un conjunto de docentes de la Facultad de Ciencias Políticas y Administrativas de la Universidad Nacional de Chimborazo (Unach), desarrollan una serie de reflexiones teóricas como empíricas, respecto a procesos de producción pública o privada, en el marco de la gestión del conocimiento.

Por ejemplo, en el capítulo: Tomando decisiones gerenciales con el apoyo de las tecnologías de la información, los autores resaltan cómo la gestión de la información se ha introducido en las organizaciones ecuatorianas de la mano de los desarrollos tecnológicos. Los 
avances en las tecnologías de la información proporcionan a las organizaciones soluciones concretas que les permiten recopilar y reutilizar su información. La gestión del conocimiento y del capital intelectual ha desarrollado nuevas áreas de la técnica como la inteligencia empresarial, el big data, que ofrecen a las organizaciones herramientas para poder implementar sistemas de información que refuerzan sus estrategias y acciones en la administración. Estas nuevas herramientas han pasado de ser poco conocidas, a ser básicas al momento de implementar mejoras y crear ventajas competitivas en la gestión. Sin duda la democratización del internet, y de diversas aplicaciones, interfaces y demás herramientas disponibles para extraer y procesar datos, han modificado los sistemas de decisiones en las organizaciones públicas y privadas.

En la actualidad son amplias las opciones de software y soluciones para la recopilación, análisis y consolidación de datos, de manera que organizaciones e instituciones cuentan con más herramientas para la toma de mejores decisiones. También es cierto un necesario replanteamiento del mercado de trabajo y la demanda de un nuevo perfil de profesional que tenga la capacidad de crear valor a partir del entorno, mediante la identificación y perfeccionamiento de los flujos de información. De nada sirve la tecnología si no se tiene el talento para poder generar conocimiento a través de ella.

Por su parte en el capítulo: Encuesta de opinión para la evaluación de la gestión pública en la ciudad de Riobamba al 2018, se discute la necesidad de captar y analizar los cambios en los entornos de las organizaciones públicas y privadas, a fin de optimizar sus facultades de cambio y adaptación. Esto es especialmente cierto para las instituciones públicas, en un marco de legitimidad democrática y de eficacia en la concesión de derechos. El interés por conocer la opinión pública es consistente con las necesidades 
de ampliación de la participación ciudadana, la nueva gestión pública, la calidad en la ejecución pública y los procesos de reforma y modernización de la administración.

A la larga, y como lo señalara Amartya Sen, existe una conexión profunda entre información pública y el bienestar social. La gestión de la información y un correcto funcionamiento público se encuentran directamente relacionados. Ocultar o negar la información es el ingrediente siempre presente en la deficiente gestión pública y en los desastres sociales. La información y el conocimiento movilizan a las instituciones y fuerzas sociales, especialmente en situaciones de emergencia o moralmente inaceptables.

De este modo, una de las responsabilidades del sector público es procurar que la información fluya en la sociedad. Generar información veraz, adecuada, al alcance de las personas mediante canales permanentes y abiertos. La disponibilidad de instrumentos y garantías públicas de acceso a la información, cubren necesidades democráticas, garantizan derechos y proveen un factor económico fundamental. La administración pública debe tener como parte de sus instrumentales las encuestas y estudios de opinión. Cada vez son más gobiernos que usan estas herramientas de indagación como parte de sus mecanismos de participación ciudadana. Recabar las opiniones de los habitantes sobre el estado de la ciudad, de la región o del país, les coloca en un papel más activo, al identificar puntualmente problemas, preferencias y prioridades.

Es importante resaltar que la temática de la opinión pública trae inserto una compleja interrelación de debates contemporáneos. Como lo pone de presente Aguilar (2017), a pesar de que el 
concepto de "opinión", no parece estrictamente académico, ha sido objeto de una intensa reflexión teórica. Hegel la justificaba como una institución propia del ordenamiento jurídico del Estado. Rousseau estableció una relación intrínseca entre "voluntad general" y "opinión pública"; o Kant relacionaba "uso público de la razón", "coincidencia pública", con "ley pública" (lo que debe estar jurídicamente permitido). El propio Habermas (1981) la ubica como la práctica política de la burguesía emergente de los siglos XVII y XVIII. Para este autor, es posible identificar un "espacio público", en el que se desarrolla la vida social, y en el cual se construye la opinión pública. Es público en la medida que los sujetos se reúnen libremente con la garantía de poder manifestar libremente su opinión, a debatir respecto de intereses generales. En caso de que el público sea amplio, se requiere de los medios de comunicación, que se convierten en los medios del espacio público.

Actualmente son múltiples preguntas que surgen respecto a la opinión pública: ¿Puede entenderse a la opinión pública como un actor social más? ¿Es un actor político o es la mera suma de opiniones particulares? ¿Participa la racionalidad en su conformación? ¿Cuáles serían las características plausibles de un debate público? Lo que sí está claro es que la información que se genera activa y discursivamente en el espacio público, se debe contar con una estructura interpretativa que le permita transformar la información pública colectada, en conocimiento público.

Como se indica en el capítulo, un observatorio puede ser una estructura funcional para la colección e interpretación de la información pública. Un observatorio es un instrumento de permanente medición e investigación de indicadores sociales, que permite detectar, analizar y difundir lo que sucede en un ámbito 
social, y que se espera esté al servicio de las políticas sociales. Es un complejo de actividades y productos que toma diferentes modalidades y dimensiones, dependiendo de los objetivos y necesidades en materia social, y con sostenibilidad de largo plazo. Se trata ante todo de un conjunto dinámico de actividades y productos. Para el caso de Riobamba, se ha implementado el Observatorio Económico de la carrera de Economía de la Universidad Nacional de Chimborazo (Unach), para de manera semestral, producir diversa información pública, y especialmente, medir las percepciones que tienen los ciudadanos respecto a los servicios públicos de la ciudad.

En el capítulo The Ecuadorian flower cluster, se reseña otra institución que gestiona información y procesos productivos: los clústeres. En términos de mercado global, la información puede ayudar a identificar ventajas competitivas y convertirse en un elemento clave para la supervivencia de organizaciones productivas en el contexto mundial. La posibilidad de comparar capacidades y condiciones que tienen países $\mathrm{u}$ organizaciones amplía la posibilidad de ubicarlos en los procesos económicos adecuados. Es importante resaltar que la información no es un mero costo de transacción, sino un factor que en sí mismo hace más eficiente a las organizaciones. De este modo, la información se han vuelto un factor crítico para las economías insertas en la globalización.

Por ejemplo, agrupar empresas en torno a un proceso productivo, bajo una matriz geográfica es en la actualidad una estrategia comprobada para la proyección internacional. Ahora bien, la constitución de clústeres como conglomerados eficientes y eficaces, que mejoran las ventajas competitivas de las organizaciones involucradas, requiere un proceso comunicativo y de transmisión de información, sin el cual no es posible la actuación conjunta. Un 
clúster en su visión tradicional se sustenta, ante todo, en procesos de complementariedad, que buscan economías de escala y eficiencia colectiva. Sin duda es un proceso de fortalecimiento de relaciones en todo sentido (económico, comercial, logístico, etc.), cuyo éxito surge de la intensidad de las relaciones que consolidan entre los agentes.

Comunicaciones horizontales y verticales, locales y no locales, en diversas redes, así como el grado de apertura de las organizaciones participantes, posibilitan el flujo de información y de conocimiento que guían una actuación conjunta. Sin este flujo de información es muy difícil que un grupo florezca y se mantenga como tal. Se requieren canales institucionales, donde los datos y su procesamiento fluyan, a la vez que se fortalecen los lazos de intercambio. Se destaca que los clústeres son fenómenos multidimensionales, compuestos por un conjunto diverso y plural de relaciones: comerciales, mercadológicas, tecnológicas, sociales, culturales, etc. que se generan y especifican en una profunda influencia cognoscitiva.

Se aborda en el texto presentado en este libro, la idea que la mera proximidad o similitud entre las organizaciones es una condición necesaria, pero no suficiente para la conformación de clústeres en la producción de flores en el Ecuador. Es múltiple la literatura que destaca como la simple aglomeración de actores no genera un clúster. Se requiere que estas empresas construyan interacciones y complementen sus actividades en un marco de intensa transmisión de información.

En el último capítulo: Los ciclos económicos. Un análisis microeconómico, se inicia una reflexión respecto de los diversos abordajes teóricos que se han presentado respecto al fenómeno de los ciclos económicos. Se vislumbra como comportamientos micro 
relacionados con la información, generan ciclos de crecimiento económico más largos, o con determinadas características. Del amplio repaso que se realiza a las distintas teorías y escuelas que han abordado el fenómeno de los ciclos económicos, es posible vislumbrar cómo la innovación (por ejemplo, el desarrollo en las tecnologías de la información o del transporte) tiene una fuerte relación con el comportamiento de la productividad.

En este marco, autores como Innis (1950) relacionan el desarrollo de tecnologías de la información, con el comportamiento de los ciclos económicos en el largo plazo. En una primera fase de expansión, la información y sus tecnologías incentivan la actividad innovadora y la productividad. En una segunda etapa el crecimiento se estabiliza, dado que las nuevas tecnologías se difunden ampliamente. En una etapa final, las tecnologías exitosas alcanzan una posición de monopolio, lo que impulsa una fase recesiva en el ciclo.

Sin duda, la difusión de información a lo largo del tiempo, la complejidad de los sistemas de comunicación y los costos de almacenamiento, son elementos que pueden determinar el comportamiento económico general. Hay costos de almacenamiento, transmisión y decodificación de la información que deben tenerse en cuenta para entender los procesos de crecimiento concreto de un país. Sin embargo, y en el capítulo que compone este libro, se evidencia cómo las tecnologías de las comunicaciones es un elemento que está ausente en la mayoría de los modelos de crecimiento o ciclo económico.

Parecería que se obvia cómo las nuevas ideas generalmente requieren aportaciones del conocimiento existente. El crecimiento económico ocurre cuando nuevas ideas reemplazan las formas existentes de hacer las cosas. En este sentido, la naturaleza del 
cambio tecnológico dependerá en parte del medio de comunicación dominante. Las tecnologías de las comunicaciones influyen en el grado en el que las ideas se sintetizan. Son la más fundamental de las tecnologías habilitadoras en la creación de conocimiento, síntesis de ideas, y divulgación de estas.

Sobre esta base, podría describirse un ciclo de crecimiento con tres fases. Inicialmente, la tasa global de crecimiento de la productividad es baja, ya que en el procesamiento de la información (sustento de la innovación), la difusión permanece limitada. Paradójicamente, en esta misma fase se presenta lo que Schumpeter (1950) denominaría destrucción creativa. A medida que las nuevas tecnologías se adoptan, el crecimiento de la productividad se acelera. En esta fase de difusión del ciclo, aunque la tasa de mejora de la productividad parezca caer, el efecto es compensado cada vez más por el aumento en la proporción de la población que adopta las mejoras desarrolladas. En una fase final, las tecnologías desarrolladas en períodos anteriores se han difundido ampliamente. Al mismo tiempo, el número de nuevas combinaciones producidas por el conjunto inicial de nuevas invenciones ha disminuido drásticamente. Por tanto, la tasa de crecimiento vuelve a caer. 


\section{Referencias}

Aguilar Villanueva L.F. (2017) Una reconstrucción del concepto de opinión pública. Revista Mexicana de Opinión Pública, (23), 125-148.

Erickson, G. S., \& Rothberg, H. N. (2014). Strategic Innovation and Sustainable Competitive Advantage: Understanding Knowledge Assets. En ISPIM Conference Proceedings (p. 1). The International Society for Professional Innovation Management (ISPIM).

Habermas, J. (1981). Historia y crítica de la opinión pública. 2a edición. Barcelona: Editorial Gustavo Gili, S.A.

Innis, H.A. (1950) Empire and Communications. Oxford: Clarendon.

Schumpeter, J.A., 1950. Capitalism, Socialism and Democracy, 3rd ed. New York: Harper 


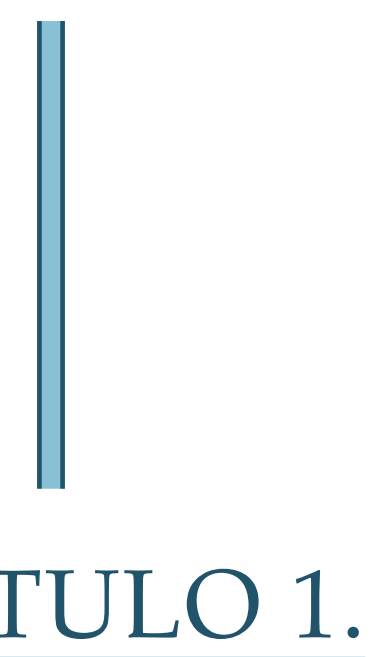

\section{TOMANDO DECISIONES} GERENCIALES CON EL APOYO DE LAS TECNOLOGÍAS DE LA INFORMACIÓN

Edinson Palacios Susana Naranjo Javier Palacios Armida Fernández 


\section{Resumen}

Las tecnologías de la información (TI) agrupan el almacenamiento, seguridad y transmisión de la información, relacionando el uso de dispositivos, redes y servicios que son de origen online con la suma de otros datos que son de procedencia offline. Las TI en las organizaciones se utilizan como medio para gestionar negocios. El crecimiento de datos, su movilidad y accesibilidad requieren un análisis y profundo estudio y tratamientos. La automatización de procesos y plataformas de información son herramientas para la toma de decisiones logrando un ahorro de recursos y tiempo. Los especialistas de las TI en las organizaciones pueden ayudar a mejorar la productividad de todas las funciones de la empresa. Se encargan del análisis, diseño, desarrollo e implementación de sistemas de información que serán consumidos por la alta gerencia para mejorar su competitividad y productividad. A pesar de todo, existe falta de conocimiento sobre las TI. Una organización que pretenda ser efectiva deberá explotar y administrar todas estas tecnologías para dar un valor agregado a toda la organización.

Palabras claves: administración, tecnologías de la información, marketing, toma de decisiones, gerencia, organización. 


\section{Introducción}

Las organizaciones cada día generan datos de fuente offline y online a través de sus productos, clientes, proveedores y competencia. Están introduciendo la tecnología para una gestión eficiente, por consiguiente, toda esa data que se está generando se convierte en información y finalmente en conocimiento para la toma de decisiones gerenciales. Anteriormente, las organizaciones consideraban como presencia electrónica, el contar con un sitio web para desarrollar su gestión y era suficiente. Para países en vías de desarrollo como Ecuador, gestionar su actividad comercial en el mercado digital aún está en desarrollo, debido a que los clientes solo generan búsquedas, no realizan una transacción comercial a través de esta. Sin embargo, las tendencias comerciales y de consumo están obligando a las organizaciones a cambiar su gestión si quieren ser competitivas.

Singh y Singh (enero de 2013) plantean que las organizaciones necesitan atraer a sus clientes potenciales a estos sitios web. Para ello necesitan saber cuántos clientes visitan su sitio web, lo que les gusta, disgusta, y establecer un sistema de inteligencia competitivo. Las organizaciones tienen establecidos sus objetivos y los caminos para lograrlos. Para esto deben ser ágiles, rápidos en reacción y efectivos, a más de desarrollar estrategias para generar experiencias con sus clientes, invertir en herramientas tecnológicas para reducir costos operativos de producción y comercialización, mejorar la calidad de productos y finalmente generar valor a los mismos. Para lograr lo mencionado es necesario que reinventen su modelo de negocio; combinando los métodos tradicionales de gestión con las herramientas de las Tecnologías de la Información (TI). Quizás sea lo más acertado para esta época. Esta integración de componentes secuenciados generará cambios, ingresos y experiencias. 
Las organizaciones en los datos tienen un activo contable, pudiendo ser estos históricos como los que se están generando día a día. Las Tecnologías de la Información (TI en adelante) evolucionaron al punto que lograron desarrollar herramientas claves para la gestión empresarial. Las aplicaciones (app) se van adaptando y generando para cada uno de los departamentos de las organizaciones y consecutivamente un macrosistema de datos. Esta investigación tiene como finalidad generar un breve análisis de las TI que están marcando el escenario actual de mercados y su empleo para tomar decisiones gerenciales desde las ciencias administrativas.

McKinsey y Company (marzo de 2017) proponen cinco enfoques y capacidades, con el fin de enrumbarse en la era del conocimiento que genera información en tiempo real. El modelo operativo propuesto para el mundo digital genera una sinergia competitiva con enfoque de valor hacia el cliente. Por otra parte, Van der Aalst (2014) realiza una propuesta para identificar el origen de la generación de información proveniente desde la red y asociado al término Internet de eventos (IoE). Para esto estableció tres categorías: 1) Internet de contenidos (IoC), 2) Internet de las personas (IoP) y 3) Internet de las cosas (IoT). Lemus y Pérez (2020) incluyen al Internet de las localizaciones (IoL), por el acceso cada vez mayor a los dispositivos móviles inteligentes que generan información geoespacial. 
Agilizar los procesos y minimizar el desperdicio
Digitalizar la experiencia del cliente y las operaciones diarias

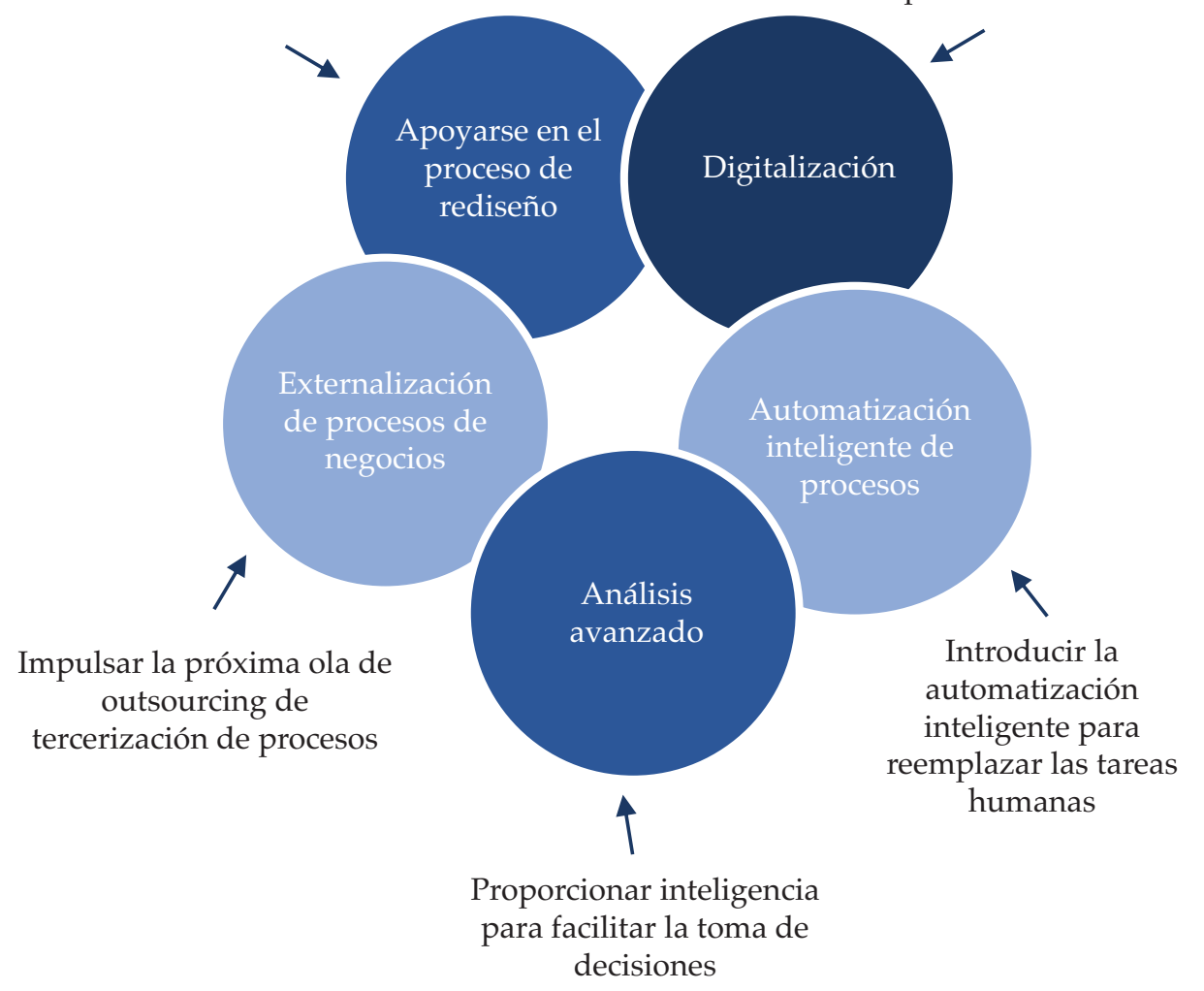

Figura 1. Cinco enfoques y capacidades para impulsar el modelo operativo de la próxima generación

Fuente: McKinsey y Company (marzo de 2017).

Hoy las organizaciones poseen gran cantidad de datos de todo tipo y de diferentes fuentes, algunos son históricos mientras otros se generan en tiempo real, unos son propios y otros son extraídos de otras fuentes de información que pueden ser externas a la misma organización. Las computadoras han posibilitado que las empresas, el gobierno y otras organizaciones almacenen y procesen grandes cantidades de datos (Freund, Williams y Perles, 1990). Los datos almacenados no eran vistos como fuentes de información para extraer patrones de comportamiento de sus clientes (Jiménez, 2017), ahora el paradigma ha cambiado ya que 
estos datos deben ser revertidos para la generación de beneficios para las organizaciones.

Dentro del amplio campo de la mercadotecnia, obtener información de una muestra y ajustarla a la población por medio de fórmulas estadísticas fue la herramienta útil para tomar decisiones, sobre todo si se habla de lanzar un producto al mercado, identificar las necesidades de los consumidores, investigar el posicionamiento de marca, entre otros. Hoy pasa de recoger muestras para analizar a las personas, dada la presencia de los mercados online, siendo estas organizaciones grandes o pequeñas que utilizan el businessto-business (B2B) y el business-to-consumer (B2C) en su gestión.

Los clientes potenciales como reales están generando grandes cantidades de datos cada momento, gracias al desarrollo tecnológico logrado en todos los campos en los últimos tiempos que ha dado el origen al Big Data (BD), Inteligencia Artificial (IA), Machine Learning (ML), Business Intelligence (BI) entre otros. Ahora se puede procesar grandes cantidades de datos en tiempo real, por medio de algoritmos matemáticos y estadísticos, estableciendo acciones de optimización para la alta gerencia.

\section{Big data}

Los clientes han evolucionado a medida que la tecnología lo ha hecho, sus gustos y comportamientos no son los mismos de hace dos o tres décadas. Producto del abaratamiento de la conectividad, la masificación de dispositivos electrónicos, la incursión de organizaciones a entornos tecnológicos y la generación de aplicaciones para ofertar bienes y servicios, las organizaciones están empleando tecnologías en su modelo de gestión que no conocen de fronteras, idiomas, religión o afinidad política; para ellas, nace la necesidad de reinventar su forma de gestionar, su 
trato con los clientes, personalizándolo, es por tal circunstancia, que las empresas tienen nuevos requerimientos para analizar sus datos, por medio de análisis predictivos, clusterización de clientes llevados al business intelligence y que se concreta con el big analytics la cual viene a convertirse en la última tendencia para negocios.

El big data viabiliza el entendimiento y la toma de decisiones para aquellos que están al frente de las organizaciones. Las grandes cantidades de datos estructurados, no estructurados y semiestructurados resultarían imposibles de analizarlos por su volumen, costos y variabilidad. En la Tabla 1, se muestra cómo evolucionan las medidas de información:

Tabla 1. Unidades de medida de datos

\begin{tabular}{|c|c|}
\hline Unidad & Equivalencia \\
\hline 1 bit & - \\
\hline 1 Byte & 8 bits \\
\hline 1 Kilobyte (KB) & 1,024 Bytes \\
\hline 1 Megabyte (MB) & 1,024 KB \\
\hline 1 Gigabyte (GB) & $1,024 \mathrm{MB}$ \\
\hline 1 Terabyte (TB) & 1,024 GB \\
\hline 1 Petabyte (PB) & $1,024 \mathrm{~TB}$ \\
\hline 1 Exabyte (EB) & 1,024 PB \\
\hline 1 Zettabyte (ZB) & 1,024 EB \\
\hline 1 Yottabyte (YB) & 1,024 ZB \\
\hline
\end{tabular}

Fuente: (Barranco, 2012).

El big data no es un concepto nuevo, el mismo ha estado junto a nosotros por mucho tiempo. En los últimos tiempos su trabajo e importancia en todos los campos, especialmente en la gerencia ha sido muy notorio, por eso hoy goza de relevancia e importancia 
para todos los actores empresariales. Su término de referencia está relacionado con la informática, estadística, matemáticas e internet; sus grandes cantidades de datos están siendo procesados en el Business Intelligence (BI), Inteligencia Artificial (IA) y el Machine Learning (ML), que recurre a varias técnicas como el business analytics, data mining o text mining con la finalidad de generar patrones que brinden información a gerentes y sirvan de soporte para tomar decisiones en tiempo real.

Tabla 2. Beneficios e inconvenientes del big data

\begin{tabular}{|l|l|}
\hline \multicolumn{1}{|c|}{ Beneficios } & \multicolumn{1}{c|}{ Inconvenientes } \\
\hline Acceso rápido a los datos. & $\begin{array}{l}\text { Gestionar grandes cantidades de } \\
\text { datos. }\end{array}$ \\
$\begin{array}{l}\text { Analizar datos y presentarlos de } \\
\text { forma resumida. }\end{array}$ & $\begin{array}{l}\text { Encontrar y obtener los datos. } \\
\text { Almacenamiento de grandes } \\
\text { cantidades de datos. }\end{array}$ \\
$\begin{array}{l}\text { Datos convertidos en } \\
\text { información y en conocimiento. }\end{array}$ & Expertos en manejo de BD. \\
$\begin{array}{l}\text { Toma de decisiones en tiempo } \\
\text { real. }\end{array}$ & Experticia en el manejo de datos. \\
\hline
\end{tabular}

Fuente: elaboración propia.

El nacimiento del big data generó oportunidades de negocios no solo a las organizaciones; a través de este, se han desarrollado otros giros de negocios debido a las grandes cantidades de datos que se manipulan, por ejemplo, el cloud computing, donde grandes organizaciones como Amazon, Oracle, Google o IBM prestan sus servicios de alojamiento. El campo de aplicación del big data es infinito como en el marketing, logística, gestión de recursos 
hídricos, estudio de lenguaje humano, genómica, investigación científica entre otras (Serrano, 2013).

Las organizaciones están adoptando estos conceptos por su utilidad en el análisis de datos donde por medio de la tecnología, sistemas, prácticas, metodologías y aplicaciones que analizan los datos de las empresas, permiten a los gerentes desarrollar un análisis crítico, comprender el negocio, identificar las oportunidades del mercado, crear negocios oportunos, en otras palabras, tomar mejores decisiones para las organizaciones (Chen, Chiang y Storey, 2012).

El big data genera muchas respuestas a las organizaciones $q u$ no sabían que lo poseían, sirviendo como puntos de referencia a los ejecutivos por la información obtenida. Estos datos por su utilidad pueden ser moldeados, probados y presentados en dashboard para el gerente, haciendo simulaciones y pronósticos en tiempo real dando soluciones eficientes que incrementen su productividad y rentabilidad. Un claro ejemplo en la utilización del big data lo presenta Power Data (2018) en un análisis de sentimiento de perpeción generado por la empresa y la marca; para ello, considera necesario conocer a la perfección al producto, sus canales de comercialización, su segmento de mercado, las campañas de marketing, entre otros aspectos, obteniendo valiosa información detrás de los datos.

Otra herramienta importante para investigar es el dark data, conocido también como datos ocultos, que no tiene origen en dispositivos electrónicos, redes o de procesos industriales. Los dark data son originarios de infraestructuras de telecomunicaciones, navegaciones, localizaciones de transporte de GPS, señales de sensores de máquinas industriales, aparatos de salud, conversaciones grabadas, correos electrónicos, chats de mensajes 
con clientes, transacciones financieras, información de campañas de marketing digital, tasas de pago por clic (CPC), SEO (Search Engine Optimization / posicionamiento natural), seguimientos de marcas entre otros. Gartner, citado por IBM (2016), define que el dark data es el "activo de información que las organizaciones recopilan, procesan y almacenan durante las actividades comerciales regulares que no se usan para análisis, relaciones comerciales y monetización directa". Posiblemente los datos generados en las organizaciones son tomados como irrelevantes, esto dado que se ignora el uso que se puede dar a los mismos y la utilidad que genera para la toma de decisiones.

IBM - Datawatch (2016) realiza una recomendación sobre cinco tipos de datos que las organizaciones deben investigar para tomar decisiones efectivas: (1) Tendencias de los datos de ventas a lo largo del tiempo, (2) datos de segmentación, (3) análisis integral de gastos, (4) puntos de referencia competitivos, (5) tendencias de investigación del mercado y la industria.

\section{Inteligencia artificial}

McKinsey y Company (s.f.) definen a la inteligencia artificial como "la capacidad de una máquina para realizar funciones cognitivas que se asocian con las mentes humanas, como percibir, razonar, aprender, interactuar con el entorno, resolver problemas e incluso ejercitar la creatividad". El futuro de las organizaciones estará en las máquinas donde ellas tomarán las decisiones (marketingdirecto. com, 2018) en lugar que las personas. Las máquinas tendrán la capacidad de automatizar la información, aprender sobre los clientes reales como potenciales y generar las mejores estrategias de comercialización para satisfacer sus necesidades.

Hay que tomar en consideración que la inteligencia artificial (IA) 
no será una herramienta opcional para los negocios. Actualmente las organizaciones no tienen las habilidades o la experiencia adecuada para utilizar este tipo de tecnología, esto según el estudio realizado por Forrester Consulting (2017) "The Next Wave of Digital Marketing is Predictive". Las empresas afrontan retos en la nueva economía digital que tiene una visión futurista, pero que requiere su aplicabilidad en el presente. Los datos generados por las actividades comerciales requieren de mucho análisis minucioso para obtener mejores resultados para las organizaciones. Según la investigación realizada por iProspect (2018) a 250 firmas influyentes a nivel mundial; se destacan las recomendaciones para gerentes y así lograr organizaciones competitivas, siendo estas: 1) Fortalecer la experiencia del consumidor, 2) asistentes digitales son los nuevos gatekeepers, 3) la inteligencia artificial y el machine learning, finalmente, 4) el comercio online omnipresente.

Las plataformas sociales, las geoetiquetas, los motores de búsqueda, las búsquedas de imágenes a través de palabras claves, los lens de pinterest, las búsquedas de voz, entre otros, permiten obtener datos de los clientes, conocimientos profundos y significativos con la finalidad de desarrollar contenidos inteligentes y eficientes para interactuar y generar beneficios a las organizaciones, vinculando cada vez más al cliente con la empresa y generando un sentimiento de lealtad hacia la marca.

Amazon es una de las organizaciones que está siendo pionera en el uso de tecnologías relacionadas con la inteligencia artificial; un claro ejemplo que está dando la vuelta al mundo es el denominado Amazon Go, donde transforma la manera de comprar en un pequeño supermercado. Para lograr esta tarea posee una infraestructura tecnológica que es el pilar fundamental de este proyecto pionero. La tienda cuenta con cámaras y sensores de movimiento detectando los productos que adquiere y aquellos 
que son devueltos a su stand, también los localiza mediante inteligencia artificial, la tienda está en capacidad de obtener datos y lecturas del cliente, aprender para futuras compras, no cuenta con personal humano de atención ya que, al terminar la compra, el cliente recibe en su teléfono móvil su factura (Marketing Directo, 2020). Ahora, nacen varias interrogantes como ¿qué harán los competidores?, ¿las máquinas terminarán remplazando a los seres humanos?, ¿cómo se brinda un servicio al cliente en función de una duda?, ¿en caso de reclamo del producto a quién se puede acudir?, ¿cuál es la experiencia que tienen los clientes al adquirir sus productos de esta manera?, entre muchas más donde el factor humano es necesario.

Se puede mencionar que la inteligencia artificial es una derivación del machine learning; otros autores, plantean que estas dos son lo mismo. Para este trabajo se toma por separado cada una de ellas con la finalidad de identificar las orientaciones que poseen y delinear sus campos de empleabilidad para las organizaciones. Una de las actividades que realiza la inteligencia artificial es el reconocimiento de patrones, toma de decisiones, entre otros. Antes esta era solo de uso para la academia. Actualmente cada vez más se está usando en el campo empresarial y se están generando adaptaciones por su utilidad para analizar los datos. Por ejemplo, los vehículos autónomos que son capaces de manejarse solos e incluso están en la capacidad de estacionarse sin la necesidad del conductor, la asistencia de voz incorporada en smartphones, calificaciones de crédito para identificar los candidatos idóneos para asignar un crédito financiero, soporte técnico en línea, reconocimiento de imagen, software intuitivo, aplicaciones móviles con mejoras para facilitar una experiencia diferente al consumidor. 
La robótica por medio de la inteligencia artificial ganó mucho espacio, debido a que logró crear herramientas para cubrir las necesidades internas de las organizaciones, como el manejo de inventarios por medio del mapeo, la sustitución de códigos de barras por chips de radiofrecuencia que emiten señales y que por medio de sensores indican el consumo de este. Dentro de las organizaciones, los robots están impulsando las marcas en stands en ferias de exposición comercial, donde se presentan nuevos productos. También se están desarrollando robots humanoides, nanotecnología, realidad aumentada y realidad virtual que impactan cada vez más a la sociedad.

\section{Machine learning}

El objetivo fundamental del machine learning es dotar a las computadoras de la capacidad de aprender, sin la necesidad de programarla constantemente. Este término tampoco es nuevo, el mismo fue utilizado en los años 50, al igual que los anteriores está cobrando importancia en todos los campos incluido el empresarial, su casuística se debe a la gran cantidad de ordenadores y dispositivos electrónicos que generan grandes cantidades de datos que requieren ser analizados por las organizaciones; adicionalmente los patrones de consumo y búsqueda de información cambiaron notablemente.

El aprendizaje automático o machine learning nace del aprendizaje de grandes cantidades de datos por medio de algoritmos detectando patrones, haciendo predicciones y recomendaciones, procesando datos y experiencias. En lugar de recibir instrucciones explícitas de programación se están usando algoritmos con la finalidad que las máquinas entiendan los gustos, preferencias, necesidad, patrones de consumo de los clientes. (McKinsey y Company, s.f.). Para algunos autores se considera como una 
subdivisión de la inteligencia artificial. Está asociada a la inteligencia humana del aprendizaje (Marketing Directo, 2018). Sin el uso de esta herramienta no se podría mejorar la experiencia del cliente, ni tampoco optimizar la logística como ya lo vienen realizando organizaciones como Amazon, por ejemplo.

En la Tabla 3, se muestra una serie de algoritmos de machine learning que son empleados en el campo comercial y que son presentados en cuadros de mando o también conocidos como dashboard.

Tabla 3. Algoritmos de machine learning para usos comerciales

\begin{tabular}{|c|c|c|}
\hline $\begin{array}{l}\text { Aprendizaje } \\
\text { supervisado }\end{array}$ & $\begin{array}{l}\text { Aprendizaje no } \\
\text { supervisado }\end{array}$ & Aprendizaje reforzado \\
\hline \multicolumn{2}{|c|}{ Algoritmos / caso de uso empresarial } & Casos de uso empresarial \\
\hline $\begin{array}{l}\text { Regresión lineal } \\
\text { Regresión logística } \\
\text { Análisis } \\
\text { discriminante lineal } \\
\text { / cuadrático } \\
\text { Árbol de decisión } \\
\text { Naive Bayes } \\
\text { Máquinas de } \\
\text { soporte vectorial } \\
\text { Bosques aleatorios } \\
\text { AdaBoost (estimulo } \\
\text { adaptativo) } \\
\text { Potenciación del } \\
\text { gradiente } \\
\text { Red neuronal } \\
\text { simple }\end{array}$ & $\begin{array}{l}\text { K-medidas de } \\
\text { agrupamiento } \\
\text { Modelo de mezclas } \\
\text { gaussianas } \\
\text { Análisis de } \\
\text { agrupación } \\
\text { jerárquica } \\
\text { Sistema de } \\
\text { recomendación de } \\
\text { contenidos }\end{array}$ & $\begin{array}{l}\text { Sistematizar las estrategias } \\
\text { de negocios y su portafolio } \\
\text { Balancear la carga en ciclos } \\
\text { de demanda variables } \\
\text { Inventario y selección de } \\
\text { inventario utilizando robots } \\
\text { Optimizar el control } \\
\text { de mando y cuadro de } \\
\text { comportamiento } \\
\text { Optimizar precios en } \\
\text { tiempo real para subasta de } \\
\text { productos con suministros } \\
\text { ilimitados en línea }\end{array}$ \\
\hline
\end{tabular}

Fuente: elaboración propia con base en McKinsey y Company (s.f.). 
Las técnicas de machine learning abarcan un campo amplio y extenso son la base fundamental del big data. Los algoritmos seguirán desarrollándose para generar aplicaciones que permitan a las organizaciones resolver problemas y seguir incrementando su cuota de mercado impactando significativamente en las decisiones estratégicas y dotando de información a los gerentes. Una de las características principales del machine learning es el aprendizaje en base a su propia experiencia, mientras más experiencia tiene, realiza mejor su trabajo. La experiencia son los datos que generan los clientes. Entonces la tecnología se pone al servicio de los clientes de una manera rápida y precisa; indirectamente al emplear estas herramientas los modelos de negocios se diversifican y generan una experiencia satisfactoria en su mercado objetivo, atrae a sus clientes potenciales y maximiza los beneficios organizacionales.

El machine learning posee muchas categorías de aprendizaje sobre las cuales las organizaciones deben establecer estrategias para lograr los objetivos esperados, dentro de estos están los aprendizajes supervisados, no supervisados, semi supervisados, basados en recompensas, extrapolación de aprendizaje, muestras en base a criterios entre otros. Algunas organizaciones que acogen el desarrollo y uso de estas tecnologías ya están trabajando en diferentes niveles como: Google, Amazon, Facebook, IBM, Toyota, Microsoft, Banco Santander, BBVA, Apple entre otros. Otro sector donde se está empleando machine learning es el industrial, en lo que hoy se denomina Industria 4.0, por ejemplo, el mantenimiento preventivo, máquinas con sensores que generan datos y que predicen cuándo debe generarse un mantenimiento o reemplazo de piezas. Con toda esta información los gerentes tienen la posibilidad de planificar eficientemente sus procesos productivos y establecer estrategias de abastecimiento, logística, reemplazo y operación atendiendo así sus mercados. 
Por último, se analizará una subderivación del machine learning que está ganando espacio y brinda nuevas oportunidades a las organizaciones, este es el deep learning o aprendizaje profundo. Este se basa en una estructura cerebral donde por medio de un computador se intenta emular al pensamiento de un humano. La principal diferencia en función del machine learning está en que a medida que aprende, extrae información y clasifica automáticamente.

Tabla 4. Algoritmos de deep learning para usos comerciales

\begin{tabular}{|c|c|}
\hline $\begin{array}{l}\text { Red neuronal } \\
\text { convolucional }\end{array}$ & Red neuronal recurrente \\
\hline \multicolumn{2}{|c|}{ Casos de uso comercial } \\
\hline $\begin{array}{l}\text { Diagnosticar enfermedades } \\
\text { a partir de exploraciones } \\
\text { médicas. } \\
\text { Detectar la marca de una } \\
\text { empresa en redes sociales, } \\
\text { para comprender su } \\
\text { dinámica. }\end{array}$ & $\begin{array}{l}\text { Generar informes de análisis para } \\
\text { operadores de valor. } \\
\text { Proporcionar traducciones de lenguaje. } \\
\text { Cambios visuales en áreas después de } \\
\text { desastres, con el fin de evaluar posibles } \\
\text { reclamos de daños (en conjunto con CNN). }\end{array}$ \\
\hline $\begin{array}{l}\text { Comprender la marca } \\
\text { desde la perspectiva } \\
\text { del cliente a través de } \\
\text { imágenes. } \\
\text { Detectar productos } \\
\text { defectuosos en la línea de } \\
\text { producción a través de } \\
\text { imágenes. }\end{array}$ & $\begin{array}{l}\text { Evaluar la probabilidad de un fraude } \\
\text { transaccional con tarjeta de crédito. } \\
\text { Generar caracteres y subtítulos para } \\
\text { imágenes. } \\
\text { Generar chatbots que buscan abordar las } \\
\text { necesidades y consultas más frecuentes de } \\
\text { los clientes. }\end{array}$ \\
\hline
\end{tabular}

Fuente: elaboración propia con base en McKinsey y Company (s.f.). 


\section{Business intelligence}

Otra de las tecnologías de la información empleada en el ámbito gerencial es el business intelligence; esta toma los datos como materia prima para generar información. El reto está en convertir esta información en conocimiento, para que los gerentes optimicen sus decisiones y generen beneficios, reduzcan costes, realicen proyecciones financieras, planifiquen ventas, establezcan eficiencia productiva, gestionen inventarios, entre otros (Varela, 2018). La inteligencia de negocios es considerada como el campo de aplicación con facultades cognitivas humanas y tecnológicas capaces de gestionar y brindar soporte en la toma de decisiones en referencia a los problemas que se presentan en los negocios (Ranjan, 2009).

Para Jiménez, Burke \& Rodríguez (2017) “business intelligence es un proceso para analizar datos para ayudar a los gerentes y otros usuarios finales a tomar decisiones más informadas, basadas en el uso de la información con diagnósticos más precisos y soluciones más inteligentes". El business intelligence no se limita solo a los campos antes mencionados, sino que genera operaciones avanzadas conocidas como business analytics para la extracción de datos, análisis predictivo, extracción de texto, análisis estadístico y análisis de big data para el descubrimiento del conocimiento.

Miah (2014), citado por Shaheb, Shah \& Shahadat (2017), afirma que el "business intelligence es una aplicación liderada por el sistema de información que integra el proceso y la tecnología para llevar a la toma de decisiones para los gerentes y los usuarios finales". El mismo autor hace una referencia a lo expuesto por Singh, H. \& Singh, B. (enero de 2013) donde manifiesta que el business intelligence utiliza ciertas tecnologías para integrar el registro de datos históricos y actuales, síntesis, transformación de datos en 
información, generación de conocimiento y su explotación en la toma de decisiones para mejorar la potencia del negocio. A continuación, se presenta una serie de funciones habituales que realiza el business intelligence y que son empleadas en la gestión empresarial.

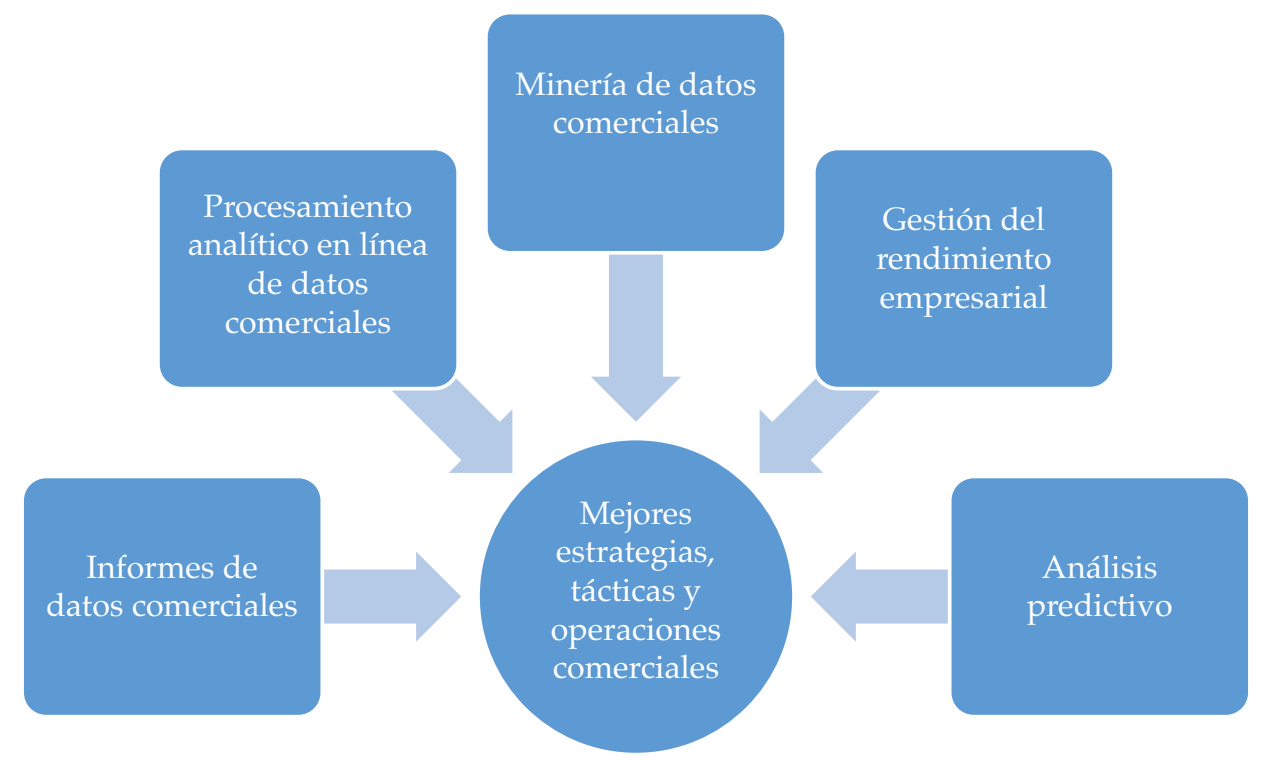

Figura 2. Funciones del business intelligence

Fuente: elaboración propia con base en Singh, H. y Singh, B. (2013).

En cambio, en la Figura 3, se definen algunos campos de aplicación del business intelligence para las organizaciones: 


\section{Medición}

Programas que crea una jerarquía de métricas de rendimiento y benchmarking, genera informes a los gerentes sobre el progreso de objetivos del negocio.

\section{Analítica}

Programa que construye procesos de negocios cuantitativos para llegar a tomar decisiones óptimas y generar un conocimiento del negocio, implica minería de datos, análisis estadísticos y análisis predictivo.

\section{Informes}

Programa que construye una infraestructura para informes estratégicos; frecuentemente involucra visualización de datos, sistemas ejecutivos de información.

\section{Colaboración}

Programa que obtiene diferentes aspectos tanto dentro y fuera del negocio para trabajar por medio del intercambio de datos y datos electrónicos.

\section{Conocimiento}

Programa para impulsar los datos de la empresa, creando, representando, distribuyendo y permitiendo las ideas y conocimientos en el negocio.

Figura 3. Aplicaciones del business intelligence

Fuente: elaboración propia con base en Singh, H. y Singh, B (2013).

Morales (2017), por ejemplo, empleó herramientas de business intelligence generando un análisis general de contenidos de investigación aplicados al sector educativo de nivel superior, contrasta herramientas tradicionales frente a técnicas de business intelligence y demuestra científicamente su utilidad, brevedad y confiabilidad, dotándole a las autoridades institucionales información precisa para tomar decisiones.

\section{Sistema de información para toma de decisiones}

Las personas en general están propensas a tomar decisiones todos los días, en las organizaciones sucede lo mismo, pero 
en esta última, una mala decisión puede marcar un antes y después. La orientación de las organizaciones está direccionada a ser productivos, generar ventajas competitivas y generar rentabilidad para la misma. Laudon y Laudon (1996), en un sistema organizacional genera una clave piramidal de información compuesta de la siguiente manera: (de abajo para arriba) sistema de procesamiento de transacciones (TPS), sistema de información gerencial (MIS), sistema de soporte a decisiones (DSS) y sistema de información ejecutiva (EIS), en donde los niveles de decisión aportan significativamente al macro nivel para el establecimiento estratégico y operacional de la organización.

"La información y los datos dependen desde la perspectiva que se miren" (Carrero, 2015), sin embargo, con el uso de herramientas de las tecnologías de la información la data trasciende al más alto nivel que es el "conocimiento", donde la implementación de tecnología transforma el quehacer organizacional, enfrentándola a nuevos retos, identificando y entendiendo patrones de consumo que las empresas no sabían que poseían y tampoco estaban en capacidad de darle lectura por la falta de estructura, conocimiento y recursos.

Lara (2015) manifiesta: "cuando se estudia la gerencia se estudia la toma de decisiones”, y contempla también el tránsito por algunas teorías como la lógica, matemática y filosófica llegando a derivar actuaciones racionales. En función de lo manifestado y captando estos aspectos en el contexto universal, se deben incluir el campo tecnológico y estadístico para obtener su utilidad.

Por medio de las tecnologías de la información la resolución de problemas no está destinados solo al cliente externo, sino también al interno, generando sistemas eficientes e inteligentes orientados a acciones concretas y latentes de la empresa. Si a este escenario 
se suma un modelo de recomendación generado por el big data $\mathrm{y}$ machine learning, los gerentes minimizan el riesgo de tomar una decisión incorrecta y maximizan la efectividad de generar soluciones que resuelvan los problemas identificados apoyado y sustentado en sus propios datos que han sido moldeados $y$ transformados en conocimiento.

El dato se convirtió en información, la información se transformó en conocimiento y el conocimiento genera competitividad y productividad. Este proceso se obtuvo de diferentes fuentes y ya no es necesario esperar tanto tiempo, ni realizar investigaciones de mercado tradicionales para identificar si un producto tuvo o no aceptación; tampoco es necesario esperar que el gerente de ventas solicite la información a sus vendedores para consolidar y presentar a la junta directiva, esto cambió cuando los datos empezaron a ser tomados como insumos de información y generaron respuestas en tiempo real. La convergencia que se ha dado al sistema gerencial inteligente con la capacidad de resolver problemas se convierte en una herramienta eficiente para el mercado y genera un ecosistema digital de decisiones confiables asistido por la tecnología. 


\section{Conclusiones}

Es fundamental que las organizaciones reinventen su modelo de negocio con enfoque hacia las tecnologías de la información para minimizar riesgos y generar valor a sus clientes; para ello, es necesario emplear algoritmos que ya se mencionaron anteriormente o a su vez desarrollar nuevos en función de las necesidades de información de las organizaciones que posean los dashboard se logrará tomar decisiones efectivas.

No solo bastará contar con un producto que cumpla los parámetros de calidad normados por los organismos nacionales o internacionales y que se reflejan en el empaque del producto; tampoco, será suficiente postear un mensaje en canales de comunicación para captar la atención de clientes, la clave estará en la orientación que las organizaciones tengan hacia los datos. Invertir en estructuras, tecnologías y colaboradores con estos tipos de conocimientos son las ventajas competitivas y comparativas que marcarán la diferencia para obtener una mayor cuota de mercado.

El acceso a las tecnologías está produciendo un fenómeno de exigencia y de valoración hacia la marca. Se debe entender que los hábitos de compra cambiaron y es muy fácil comparar marcas, precios y atributos. Los clientes poseen muchas fuentes para valorar un producto antes de su compra, entonces, las tecnologías de la información se convierten en herramientas de apoyo para conocer y predecir este nuevo comportamiento de los clientes y orientar los esfuerzos de mercado hacía lo que el cliente está buscando.

Los datos son el principal activo que tienen las organizaciones. Es importante cambiar los paradigmas y adaptarse al medio. 
Los datos generan oportunidades competitivas y orientan a la generación de experiencias diferenciadoras hacía los clientes. El conocimiento que las organizaciones puedan extraer de estos marcará la rentabilidad de esta.

Una decisión gerencial está sujeta a la información que se proporcione para llevarla a cabo. No basta generar modelos estadísticos y matemáticos eficientes si quien es el encargado de llevar a cabo las propuestas, no posee habilidades, destrezas y conocimientos para leer correctamente lo que está sucediendo en el entorno y mucho más aún, si no tiene la visión de llevar a la organización a ese futuro deseable. Para esto es necesario investigar a fondo las tecnologías de la información tomando como principio que la tecnología, el conocimiento y el ser humano que evoluciona día a día; de allí la importancia de estudiarlos y comprenderlos. 


\section{Referencias}

Barranco, R. (18 de junio de 2012). ¿Qué es Big Data? IBM developer Works. Recuperado de: https://www.ibm.com/ developerworks / ssa/local/im/que-es-big-data /

Carrero, J. (28 de septiembre de 2015). Big Data y Creatividad: Todo está cambiando. Puro Marketing. Recuperado el 21 de enero de 2018, de http://www.puromarketing.com/12/25440/bigdata-creatividad-todo-esta.html

Chen , H., Chiang, R., \& Storey , V. (2012). Business intelligence and analytics: from big data to big impact. Management Information Systems Quarterly, 36(4), 1165-1188. Recuperado de: https:/ / misq.org/skin/ frontend/ default/misq/pdf / V36I4/SI_ChenIntroduction.pdf

Forrester Consulting. (2017). The Next Wave of Digital Marketing is Predictive. Investigation, Sizmek.

Freund, J., Williams, F., \& Perles, B. (1990). Estadística para la Administración: con enfoque moderno. México: Prentice-Hall Hispanoamérica, S.A.

IBM - Datawatch. (2016, 16 de agosto). Marketing dark data: 5 dark data sources that lead to better marketing insights with IBM watson analytics and cognos analytics. Business analytics blog. Recuperado de: https://www.ibm.com/blogs/businessanalytics / the-dark-side-of-the-source-bringing-unusedcustomer-data-into-the-light /

IBM. (20 de septiembre de 2016). Dark data discovery: Improve marketing insights to increase ROI. (F. Moreno, Editor). Business analytics blog. Recuperado el 24 de Enero de 2018 de: https: / / www.ibm.com/blogs/business-analytics / darkdata-discovery-im prove-marketing-insights-to-increaseroi/

iProspect. (2018). Future Focus 2018: The New Machine Rules. Madrid: Investigation

Jiménez, C., Burke, M., \& Rodríguez, I. (2017). Statistical metadata 
in knowledge discovery. DYNA, 84(202), 270-277. DOI: http: / / dx.doi.org/10.15446 / dyna.v84n202.61417

Jiménez, R. (2017). Big Data y Machine Learning, los nuevos mejores amigos del marketing. Ctrl: control \& estrategias, pp. 72. Recuperado el 20 de Enero de 2018, de http:// controlpublicidad.com/big-data-y-machine-learningnuevos-mejores-amigos-del-marketing /

Lara, N. (2015). Decisiones complejas gerenciales. Caracas: Guardagujas.

Laudon, F. \& Laudon, J. (1996). Sistemas de Información. México: Diana.

Lemus, D. \& Pérez, R. (2020). Ciencia de datos y estudios globales: aportaciones y desafíos metodológicos. Colombia Internacional (102), pp 41-62.

Marketing Directo. (2020, 15 de febrero). Las diferentes definiciones de inteligencia artificial explican su importancia. Recuperado el 15 de febrero de 2018, de Marketing Directo.com: https: / / www.marketingdirecto.com/marketing-general/ businessintelligence-powered-by-neural-one/las-diferentesdefiniciones-inteligencia-artificial-explican-importancia.

Marketingdirecto.com. (19 de enero de 2018). Marketing Directo. Recuperado el 19 de enero de 2018, de La inteligencia artificial cambiará el influencer marketing este 2018: https: / / www.marketingdirecto.com/digital-general/digital/ inteligencia-artificial-cambiara-influencer-marketing-2018

McKinsey \& Company. (Marzo de 2017). The next-generation operating model for the digital world. Digital McKinsey. Recuperado de: https://www.mckinsey.com/businessfunctions / digital-mckinsey / our-insights / the-nextgeneration-operating-model-for-the-digital-world

McKinsey \& Company. (s.f.) An executive's guide to AI. McKinsey Analytics. Recuperado el 15 de febrero de 2018, de:

https: / / www.mckinsey.com/business-functions/mckinseyanalytics / our-insights / an-executives-guide-toai?cid=other-eml-alt-mip-mck-oth 1802\&hlkid=49ff99aec5 774678b14952672b1d0712\&hctky=10284307\&hdpid=7af816 3a-80b8-44da-9160-a3115aa11055 
Morales, C. H. (2017). SLR aplicando text mining para la adopción de software de business intelligence en el área de investigación de la UNACH. Tesis de Maestría. Recuperado el 22 de febrero de 2018, de Universidad de las Fuerzas Armadas, de: http:// repositorio.espe.edu.ec/xmlui / handle $/ 21000 / 13139$ ?locale-attribute $=$ en

Power Data. (09 de febrero de 2018). Big Data: ¿En qué consiste? Su importancia, desafíos y gobernabilidad. https://www. powerdata.es/big-data

Ranjan, J. (2009). Business intelligence: concepts, components, techniques and benefits. Journal of Theoretical and Applied Information Technology, 9(1), 060-070. http:/ / www.jatit.org/ volumes / research-papers / Vo19No1 / 9Vo19No1.pdf

Serrano, J. (2013). Big data y not so big data. Anuario ThinkEPI (1), pp. 161-163. Recuperado el 21 de enero de 2018, de https: / / dialnet.unirioja.es / servlet / articulo? codigo $=4234739$

Shaheb, A., Shah, M., \& Shahadat, K. (2017). Analysis of Interaction between Business Intelligence and SMEs: Learn from Each Other. JISTEM - Journal of Information Systems and Technology Management, 14(2), pp. 151-168. doi: http: / / dx.doi.org/10.4301/s1807-17752017000200002

Singh, H., \& Singh, B. (enero de 2013). Business Intelligence: Effective machine learning for business. International Journal of IT, Engineeri, 2(1), 13-18. https:// pdfs.semanticscholar. org / 690b / 7303e8acf5d8b638b5de389b08dfaeaebf9a.pdf

Van der Aalst, W. (2014). Data Scientist: The Engineer of the Future. Enterprise Interoperability VI, 13-28. Nueva York: Springer, Cham.

Varela, P. (2018, 22 de enero). Big Data, Dark Data... ¿dinero que las empresas tiran a la basura? Recuperado el 24 de enero de 2018, de marketingdirecto.com: https://www.marketingdirecto. com / punto-de-vista / la-columna / big-data-dark-datadinero-las-empresas-tiran-la-basura-pepe-varela. 


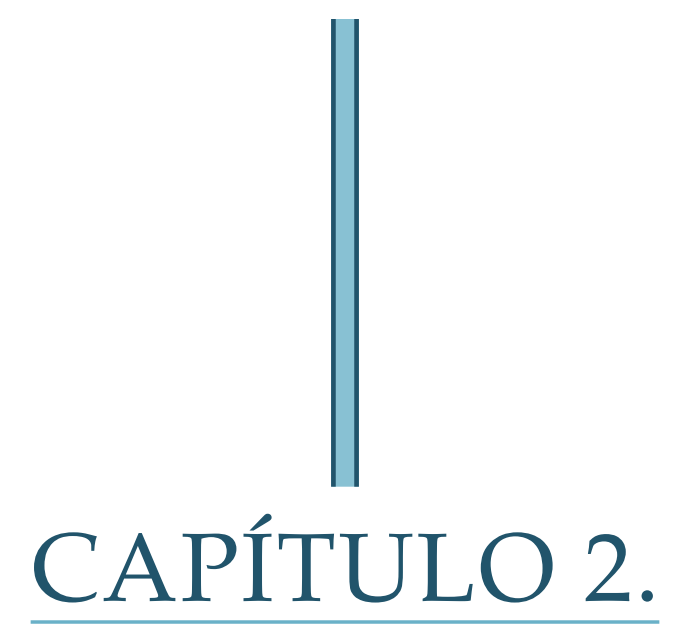

\section{ENCUESTA DE OPINIÓN PARA LA EVALUACIÓN DE LA GESTIÓN} PÚBLICA EN LA CIUDAD DE RIOBAMBA AL 2018

María G. González-Bautista María F. Cazorla-Logroño Wilman G. Carrillo-Pulgar María E. Borja-Lombeida 


\section{Resumen}

El presente trabajo explora, a través de una encuesta de opinión, la imagen de diversas organizaciones autónomas y dependientes del Gobierno, de los servicios públicos locales, de las instituciones de educación superior, y la tendencia en las elecciones a gobiernos seccionales celebradas en 2019. La validación metodológica se fundamentó en la aplicación de una escala de Likert en un diseño cuasi experimental. Como resultado de la aplicación de la encuesta se evidencia un nivel de valoración de la gestión y los servicios, tendiente a buena, y la ausencia de una tendencia marcada de decisión de voto. La imagen de las organizaciones públicas evidencia una mayor aceptación, especialmente las instituciones de educación superior, con una valoración de buena a muy buena. En la gestión de las autoridades locales y provinciales el rango de valoración se sitúa entre regular y mala. Debido a su importancia, la encuesta se repetirá para identificar variaciones temporales significativas en los resultados, mejorar su interpretación, y apoyar la toma de decisiones públicas y privadas.

Palabras clave: evaluación, encuesta de opinión, gestión pública, opinión pública. 


\section{Introducción}

La opinión pública ha tomado gran importancia en dos sentidos. El primero es la necesidad de conocer la percepción de los ciudadanos acerca de temas del ámbito político, económico y social. El segundo es transferir la información a los representantes de los sectores socioeconómicos, autoridades políticas, miembros de gobierno, representantes de gremios, empresarios, entre otros. A nivel internacional, la asociación mundial para la investigación en opinión pública (WAPOR) promueve este proceso, a través de la difusión de investigaciones, intercambios educativos, declaraciones públicas, conferencias y seminarios desarrollados en todo el mundo, WAPOR (2017). En el Ecuador, han sido las instituciones de educación superior (IES), las que han adquirido un rol protagónico a través de la transferencia de conocimientos generados. El rol de las universidades en la investigación ha tomado cada vez mayor importancia, principalmente por la imparcialidad, objetividad y confidencialidad con la que se maneja la información. En este marco, la escuela de Economía, de la Facultad de Ciencias Políticas y Administrativas de la Universidad Nacional de Chimborazo (Unach), crea el Observatorio Económico, con el objetivo de desarrollar investigación y divulgación científica económico-empresarial del cantón Riobamba y de la provincia de Chimborazo, a través de la participación proactiva de docentes, investigadores y estudiantes, como aporte para la generación de una economía sostenible, dinámica y competitiva basada en el conocimiento.

Como una de sus primeras actividades, el Observatorio realizó una encuesta de opinión para la evaluación de la gestión pública en la ciudad de Riobamba en el año 2018, que integra aspectos como: la imagen de organizaciones del Estado, los servicios prestados, las instituciones de educación superior y la tendencia 
electoral de las próximas elecciones de gobiernos seccionales. El presente trabajo exhibe los resultados obtenidos, en el marco teórico y metodológico de la opinión pública. Para cumplir con este objetivo, en una primera sección se aborda el estado del arte en cuanto a la opinión pública y su uso metodológico y científico. En una segunda se detalla la metodología cualitativa y cuantitativa utilizada. En la tercera, se exponen los resultados de la encuesta, y para finalizar, se presentan las conclusiones.

\section{¿Qué es la opinión pública? Presión social y racionalidad}

La denominación de opinión pública hace referencia a un conjunto de consideraciones, proporcionadas por la población, que tienen un criterio relevante de coincidencias. Por lo general estos criterios propios aluden a la política, la economía, y todos los temas que encierran un interés público, el cual representa a una sociedad determinada ( Raig 1999). La opinión pública, para Pareja y Echeverría (2014), puede comprenderse como un concepto que articula un fenómeno social, en que existe una serie de ideas, pensamientos, creencias, en torno a diversos temas de carácter colectivo. Sin duda, la opinión pública constituye uno de los pilares sobre los cuales los modelos democráticos contemporáneos se erigen, y es la base de distintas actividades que realiza el Estado y diversos sectores de la sociedad. La opinión pública representa el criterio y el juicio de la sociedad desde su problemática y sus tensiones (Guitiérrez, 2017).

En el libro La opinión pública en Habermas, Boladeras (2001) explica desde el pensamiento habermasiano, cómo la opinión pública tiene implicaciones y sentidos diversos, que surgen en un espacio público ilimitado, debido a la multiplicidad de intercambios sociales relacionados en su interior. La opinión pública es pieza clave de la dinámica del poder y de los procesos políticos que, en 
una propuesta de política deliberativa, busca superar los déficits democráticos de los estados contemporáneos. En el espacio público es donde surge la opinión pública, la cual puede ser manipulada y deformada, pero su importancia recae en ser el eje de cohesión social y soporte de la legitimación. Todo depende de la actividad que se dé dentro de ese espacio, para permitir las libertades individuales y políticas. Es la dinámica de lo público formado por cualquier grupo de diálogo, donde distintas personas con intereses comunes tratan cuestiones varias.

El estudio de la opinión pública ha de tomar en consideración, lo que opina la gente, los paradigmas del colectivo social acerca de los asuntos públicos, cómo son trasmitidos a los demás y cómo las opiniones que circulan entre las personas llegan a ser una parte sustancial de la realidad social. Cuando la gente habla entre sí y pone en circulación sus opiniones en el espacio social, contribuye a la formación de un tejido social específico, intangible y a la vez aprehensible para casi todos (Mora, 2005). La función de la opinión pública de acuerdo con Noelle-Neumann (1995), posee un componente moral o estético, por tanto, la espiral del silencio corresponde a fenómenos observables entre los individuos relativos a normas sociales. Asimismo, contiene un poder explicativo complejo al enlazar los niveles individual y social, incluyendo varios campos: político, social y psicológico, entre otros.

En este marco, se puede describir a la opinión pública como la opinión dominante que obliga a la obediencia de actitud y comportamiento, amenazando al individuo disidente con el aislamiento y al político con la pérdida del apoyo popular (NoelleNeumann, 1995). La causa de la transformación de la suma de las opiniones individuales en opinión pública es la continua interacción entre las personas debido a su naturaleza social. La 
amenaza de aislamiento, el miedo al aislamiento, la continua observación del clima de opinión y la evaluación de la fuerza o de la debilidad relativa de los diferentes puntos de vista, determina si la gente expresa sus opiniones o permanece callada (Noelle, 1995).

\section{Algunos problemas de la opinión pública}

Falta de competencias: se refiere a las reservas sobre la capacidad y la facilidad para opinar en los asuntos públicos. Quizá una de las posturas mayormente criticadas es la de Lippmann (1995) que afirma en su obra Public Opinion: "el mundo que debemos afrontar políticamente queda fuera de nuestro alcance, visión y comprensión" (p. 41). El ser humano forma sus opiniones con base en información incompleta, sesgada y poco confiable, ya sea por motivos propios (temores, prejuicios, ideas preconcebidas, o por contribución de los medios de difusión. La solución que el autor propone es rediseñar la gobernabilidad democrática creando gabinetes de información expertos e independientes, que tuvieran acceso sin limitaciones a todos los hechos y que hicieran inteligible toda la información. De lo contrario hay que abandonar la idea de crear una opinión pública competente.

Falta de recursos: A diferencia de Lippmann, otros autores como Dewey (1927) consideran que el problema no es la incompetencia de los individuos, sino los medios insuficientes para la comunicación pública. Para Dewey (1927) no es necesario que los individuos tengan un conocimiento completo y exacto de todo lo que sucede a su alrededor, solo bastaba con que tuviera la capacidad de juzgar el conocimiento proporcionado por los expertos en esa transmisión. La solución para este autor es ofrecer más y mejores recursos para la información y formación. 
Tiranías de la mayoría: es el peligro de que prevalezca la mediocridad generalizada en la opinión creada y mantenida por la presión de la mayoría, o lo que es lo mismo, que frente a amplias mayorías los puntos de vista de las minorías aun siendo importantes y validas, se desvanezcan. Este era el temor de Tocqueville (1835).

Susceptibilidad a la persuasión: la capacidad de los medios para despertar emociones no racionales en el público y derivarlo a determinadas posiciones o conductas.

Dominio de las elites: Otro motivo de discusión es lo que Ginsberg (1986) mencionó: la domesticación de las creencias de las masas, refiriéndose a la pasividad del público, lo que provoca el dominio por parte del Gobierno y las elites agrupadas.

La encuesta de opinión pública como instrumento de investigación

Para el sicólogo Paul Lazanfeld en su libro Personal influence: The part played by people in the flow of mass communications, los estudios de opinión se deben mejorar constantemente, a través de sus instrumentos y metodología, con base en estos criterios los resultados muestran la cambiante realidad social, considerando la probabilidad de imprecisiones dado que lo que se estudia son criterios y actitudes de la sociedad (Lazarfeld, Katz y Roper, 2017).

Como cita Heneeus (2010):

Esta técnica permite conocer la variada y riquísima diversidad de opiniones existentes en una sociedad, lo cual a menudo tiende a simplificar a través del análisis de datos objetivos, como indicadores económicos (...) por la 
importancia que han adquirido los asuntos económicos, por la consolidación de la economía y por la importancia que la prensa da a la información económica (p. 14).

A pesar de los problemas señalados, el perfeccionamiento técnico / metodológico de las encuestas, la pericia de los encuestadores, la supresión de intenciones manipuladoras, normas regulatorias/ éticas, garantizan la rigurosidad y racionalidad del instrumento. Las encuestas de opinión pública a diferencia de los sondeos de opinión se consideran instrumentos científicos, basados en procesos estadísticos. Este proceso integra acciones como: el establecer una metodología de muestreo acorde con el objetivo de la investigación, conocer el universo de estudio y determinar una muestra representativa. Su éxito, además, dependerá de otros factores como los canales o medios por los que se aplicará la encuesta, el diseño del cuestionario (objetivo, lógica, semántica) y el conocimiento de los encuestadores. La rigurosidad científica con la que se lleve el proceso permitirá que los resultados expresen la realidad (Ojeda, Gontero y Ceballos, 2010).

Para Ojeda et al. (2010) la encuesta infiere la opinión general, a partir de una parte de la sociedad, sustentada en la ley de los grandes números de la teoría de probabilidades. La repetición tendiente a infinito de un mismo proceso involucra la frecuencia de que suceda un hecho y tiende a ser una constante; es decir que, bajo esta teoría, la realidad coincide efectivamente con lo que sucede en el objeto de estudio investigado (Cuesta, 1984). La capacidad de realizar pronósticos a partir de una encuesta de opinión es otro aspecto relevante, sin embargo, posee limitaciones debido a la dependencia de la opinión dinámica y los lapsos de tiempo, con una ejecución actual, difusión posterior y predicción futura (Ojeda et al. 2010). 
Importancia, límites y excesos de las encuestas de opinión pública

Una de las contribuciones de las encuestas de opinión pública, es evaluar de forma subjetiva la gestión pública y el estado de la economía. Se dispone de valiosa información estadística objetiva de los principales indicadores económicos. Sin embargo, estos datos no muestran la percepción de los ciudadanos, requerimiento que solventan las encuestas de opinión pública. Para Heneeus (2010):

Las percepciones subjetivas de los ciudadanos no están primeramente definidas por el nivel de desarrollo de la economía según lo muestran los indicadores objetivos, sino que están influidos por las percepciones políticas e ideológicas, con las cuales los individuos filtran la información económica (p. 28).

Las encuestas de opinión poseen importancia, ya que se constituyen en una forma de expresión y para la administración pública y organismos privados, una herramienta para la toma de decisiones, a través de su aplicación se puede conocer el rango de separación entre una evaluación objetiva y subjetiva (Huneeus, 2010). Según Baró (1998), la encuesta de opinión pública es importante como instrumento desideologizado porque toma en cuenta ya no el discurso oficial, sino el discurso de la población.

Baró (1998) toma como referencia que una encuesta puede llegar a representar una cuota de poder para la población, lo que a su vez le permite enfrentar su propia imagen y contrastar entre lo que vive y lo que dice el discurso, verificar la concordancia entre la experiencia social y la personal. Castro (2012) argumenta que la construcción, aplicación y análisis de una encuesta es un proceso 
largo y delicado, se requiere definir el problema a investigar y contar con un marco teórico previo, establecer el diseño de investigación y su finalidad.

Las administraciones públicas deben tomar este instrumento como una exploración en un momento determinado de las tendencias de opinión de los ciudadanos encuestados, sin justificar como elemento determinante en sus acciones los resultados obtenidos, sustituyendo otros elementos de participación ciudadana que darán mayor imparcialidad en las políticas públicas (Ojeda et al., 2010). Generalmente se pone en tela de juicio la capacidad de las encuestas de opinión pública, de reflejar la realidad, debido a que se asocia su ejecución con la injerencia política en la difusión de los resultados, cuando se publican de manera indiscriminada e irresponsable. Adicionalmente la muestra encuestada, evidencia falta de conocimientos, unidos a la distorsión, falsedad, e invención de la información (Ojeda et al., 2010).

Las encuestas sin una base metodológica adecuada limitan la capacidad de pronóstico de la información, una encuesta sólida metodológicamente permite reflejar la opinión de la sociedad a partir de una inferencia, y el uso de la información genera un valor científico fundamental, que permita una aproximación a la verdad.

Existen estudios que analizan las distorsiones en la información, que se pueden producir a partir del uso de las encuestas de opinión pública. Bourdieu (1992) descarta tres postulados de su metodología, primero: la producción de una opinión no está al alcance de todos, segundo: las opiniones no tienen el mismo peso y tercero: el aplicar una misma pregunta, implica un consenso sobre 
el problema que en la práctica no existe; estos a decir del autor producen distorsiones a pesar de cumplir con una metodología.

\section{Imparcialidad ligada a la encuesta de opinión pública}

La divulgación de los resultados de las encuestas de opinión pública puede influir en la sociedad, en los organismos y entes evaluados. La sociedad puede modificar su criterio y las organizaciones evaluadas modifican sus estrategias y publicidad, pudiendo desencadenar en una manipulación de la opinión pública, frecuente en procesos electorales (Huneeus, 2010). Por tanto, existen algunas interrogantes alrededor de la influencia de las encuestas en la opinión de la ciudadanía, una de ellas expone que las personas se adhieren y adaptan al criterio que prevalece. De ahí la importancia de generar los procesos de investigación con responsabilidad científica definiendo claramente sus alcances y limitaciones.

Las encuestas de opinión pública poseen alta aceptación, académicos y políticos discuten constantemente sobre los alcances, metodología y resultados. Para quienes critican esta metodología, sus resultados muestran, a decir de Heneeus (2010), "intrínsecamente una simplificación, pues estudia asuntos complejos a través de preguntas relativamente simples que se formulan a la población mediante alternativas excluyentes" ( $p$. 11). En la Tabla 5, se propone a manera de resumen las ventajas y desventajas del uso del instrumento encuesta de opinión pública basado en la recopilación de varios autores. 
Tabla 5. Ventajas y desventajas de las encuestas de opinión pública

\begin{tabular}{|c|c|}
\hline Ventajas & Desventajas/límites \\
\hline $\begin{array}{l}\text { Informar sobre la opinión de la } \\
\text { sociedad. }\end{array}$ & $\begin{array}{l}\text { Manipulación de la opinión } \\
\text { pública. }\end{array}$ \\
\hline $\begin{array}{l}\text { Nexo entre las autoridades, } \\
\text { empresarios, líderes y la sociedad. }\end{array}$ & Volatilidad de las opiniones. \\
\hline $\begin{array}{l}\text { Herramienta para la toma de } \\
\text { decisiones. }\end{array}$ & Encuestadores no capacitados. \\
\hline $\begin{array}{l}\text { Expone singularidades, cambios en } \\
\text { el tiempo, distintos grupos sociales. }\end{array}$ & Sobredimensión de los resultados. \\
\hline $\begin{array}{l}\text { Control de la autoridad y } \\
\text { fortalecimiento de la democracia. }\end{array}$ & $\begin{array}{l}\text { No conocer los límites de la } \\
\text { información. }\end{array}$ \\
\hline Función de pronóstico. & $\begin{array}{l}\text { Imprudencia en la divulgación en } \\
\text { los resultados. }\end{array}$ \\
\hline Información retrospectiva. & Políticas públicas prematuras. \\
\hline Simplificación de la información. & Resultado no reflejen la realidad. \\
\hline & $\begin{array}{l}\text { Resistencia y desinterés del } \\
\text { encuestado. }\end{array}$ \\
\hline
\end{tabular}

Fuente: elaboración propia con base en Boladeras (2001), Gutiérrez (2017), Heneeus (2010), Lazarfeld et al., (2017), y Ojeda et al. (2010).

\section{Métodos cualitativos y cuantitativos}

Para el ejercicio empírico desarrollado, el método de investigación aplicado es cuasi experimental, con un muestreo probabilístico. El universo de investigación son los habitantes de la ciudad de Riobamba, con 225.741 personas, según los datos del INEC (2010). A partir del cálculo de la muestra se estableció 384 encuestas, con un nivel de confianza de $95 \%$ y un margen de error del 5\%. En la 
Tabla 6, se presenta la distribución geográfica de la muestra por parroquias urbanas, de las encuestas realizadas.

Tabla 6. Número de encuestas por parroquias

\begin{tabular}{|c|c|c|}
\hline Parroquias urbanas & Número de habitantes & $\begin{array}{c}\text { Número de } \\
\text { encuestas }\end{array}$ \\
\hline Lizarzaburu & 42595 & 130 \\
\hline Velasco & 33810 & 104 \\
\hline Maldonado & 25976 & 80 \\
\hline Veloz & 19702 & 60 \\
\hline Yaruquíes & 2724 & 10 \\
\hline Total & $\mathbf{1 2 4 8 0 7}$ & $\mathbf{3 8 4}$ \\
\hline
\end{tabular}

Fuente: elaboración propia.

En el diseño de muestra de la encuesta de opinión pública, se realiza la distribución geográfica por parroquias de las encuestas realizadas, a partir de los datos demográficos del INEC (2010).

Tabla 7. Ficha Metodológica

\begin{tabular}{|l|l|}
\hline Título del estudio. & $\begin{array}{l}\text { Encuesta de opinión para la evaluación de la } \\
\text { gestión pública en la ciudad de Riobamba al } \\
2018 .\end{array}$ \\
\hline Organismo ejecutor. & $\begin{array}{l}\text { Observatorio Económico Empresarial de la } \\
\text { Universidad Nacional del Chimborazo. }\end{array}$ \\
\hline Universo. & Habitantes del cantón Riobamba (225741). \\
\hline Tamaño muestral. & $\begin{array}{l}384 \text { encuestas en 5 zonas censales - INEC } \\
2010 .\end{array}$ \\
\hline Error muestral. & Nivel de confianza 95\%, error muestral 5\%. \\
\hline
\end{tabular}




\begin{tabular}{|l|l|}
\hline $\begin{array}{l}\text { Método de recolección de } \\
\text { información. }\end{array}$ & $\begin{array}{l}\text { Encuesta aplicada en las parroquias urbanas } \\
\text { de la ciudad de Riobamba. }\end{array}$ \\
\hline $\begin{array}{l}\text { Período de recolección de } \\
\text { información. }\end{array}$ & 18 de abril 2018. \\
\hline
\end{tabular}

Fuente: elaboración propia.

El instrumento utilizado es la encuesta, estructurada en un cuestionario de nueve preguntas de tipo abierto y cerrado. Las preguntas cerradas integran la escala de valoración de Likert y las preguntas abiertas recogen opciones de múltiple de respuesta. Para esta investigación se modificó la escala en la valoración cualitativa, manteniendo el rango de valoración cuantitativa y su metodología (Likert, 1932). Como se describe en las tablas 8 y 9:

Tabla 8. Escala tipo Likert, aplicado a la calidad y suministro de servicios públicos y la imagen de instituciones públicas y privadas

\begin{tabular}{|c|c|c|c|c|c|}
\hline Preguntas & Muy mala & Mala & Regular & Buena & Muy buena \\
\hline 1 & 1 & 2 & 3 & 4 & 5 \\
\hline 3 & 1 & 2 & 3 & 4 & 5 \\
\hline 4 & 1 & 2 & 3 & 4 & 5 \\
\hline 5 & 1 & 2 & 3 & 4 & 5 \\
\hline 6 & 1 & 2 & 3 & 4 & 5 \\
\hline
\end{tabular}

Fuente: elaboración propia.

Tabla 9. Escala tipo Likert, aplicada a la credibilidad de las autoridades de la provincia

\begin{tabular}{|c|c|c|c|c|}
\hline $\begin{array}{c}\text { Nada de } \\
\text { credibilidad }\end{array}$ & $\begin{array}{c}\text { Poca de } \\
\text { credibilidad }\end{array}$ & $\begin{array}{c}\text { Algo de } \\
\text { credibilidad }\end{array}$ & $\begin{array}{c}\text { Mucha } \\
\text { credibilidad }\end{array}$ & $\begin{array}{c}\text { Alta de } \\
\text { credibilidad }\end{array}$ \\
\hline 1 & 2 & 3 & 4 & 5 \\
\hline
\end{tabular}

Fuente: elaboración propia. 
A través de la estadística inferencial y el uso del software SPSS se procesó los datos, los resultados se presentan a través del análisis de medias (medidas de tendencia central), para determinar las tendencias subjetivas y su orientación según la escala de valoración de Likert propuesta. Se analizan las inferencias más significativas y se muestran en el apartado de resultados.

\section{Resultados y discusión}

\section{Aspectos generales}

En la encuesta realizada se determinó que el 45,05\% de los encuestados son hombres y el 54,95\% mujeres, de los cuales el $22,40 \%$ se encuentran en edades comprendidas entre 19 a 28 años, el $22,66 \%$ se encuentran en edades de 29 a 28 años, el $22,66 \%$ se hallan en edades entre 39 a 48 años, el 15,66\% en edades entre 49 a 58 años, el 10,42\% en edades entre 59 a 68 , y 6,3\% son adultos mayores de más de 68 años. El nivel de instrucción que tienen los encuestados es el siguiente: bachillerato $37,13 \%$, superior $39,84 \%$, educación básica 18,70 \%, posgrado 3,1\% y sin ningún nivel de instrucción el 1,08\%. 


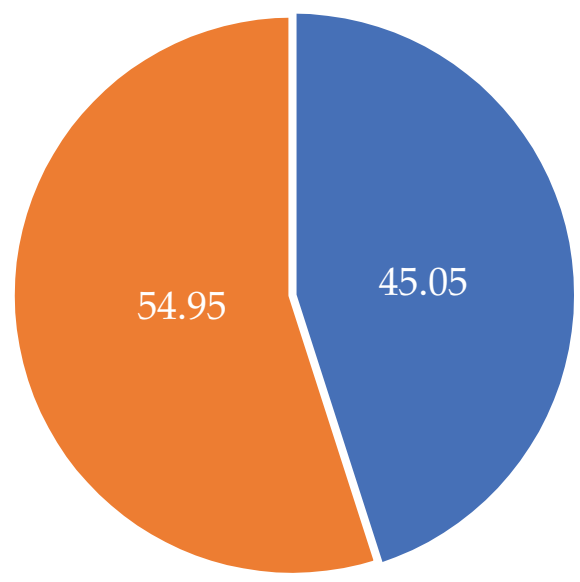

Maculino $\quad$ Femenino

Figura 4. Distribución por sexo

Fuente: elaboración propia con base en la encuesta de opinión pública aplicada en Riobamba. Abril 2018.

$\square 19$ а $28 \square 29$ а $38 \square 39$ a $48 \square 49$ a $58 \square 59$ a $68 \square$ mas de 68

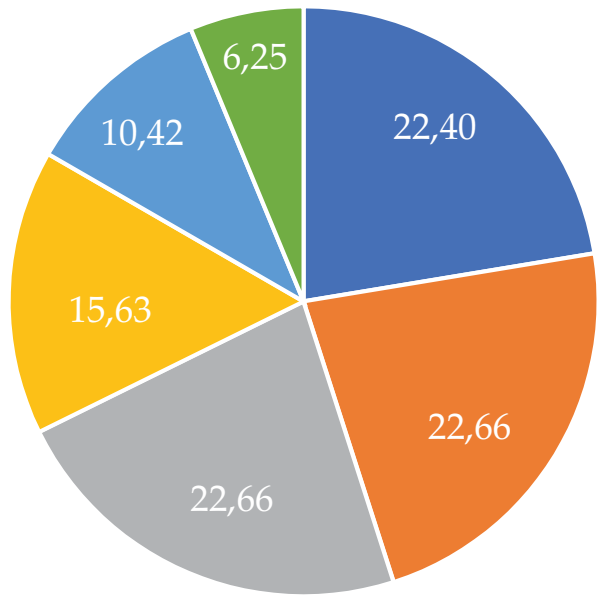

Figura 5. Edades de las personas encuestadas

Fuente: elaboración propia con base en la encuesta de opinión pública aplicada en Riobamba. Abril 2018. 


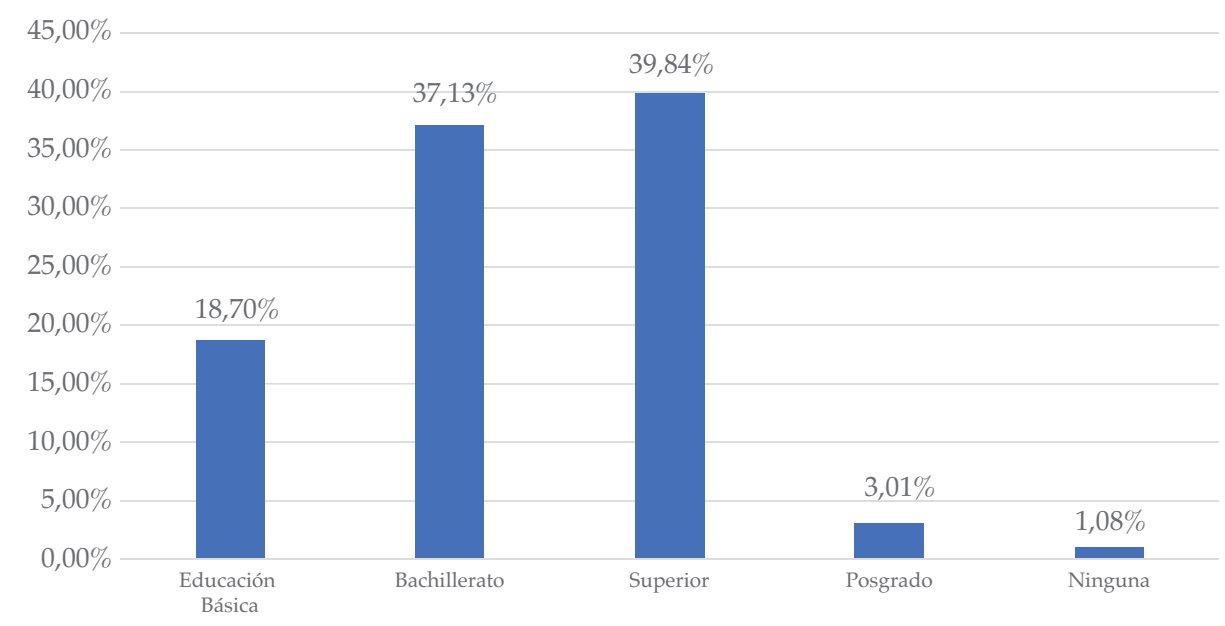

Figura 6. Nivel de instrucción de las personas encuestadas

Fuente: elaboración propia con base en la encuesta de opinión pública aplicada en Riobamba. Abril 2018.

\section{Aspectos específicos}

Imagen que tienen los riobambeños de las instituciones de la ciudad

En cuanto a la imagen que la ciudadanía tiene de las instituciones de carácter públicas y privadas de la ciudad de Riobamba, los encuestados reflejaron que, las instituciones con mayor imagen promedio son: la Empresa Eléctrica Riobamba con 3,89, Registro Civil y bancos privados 3,83, respectivamente, seguido de los medios de comunicación con 3,81, el ECU 911 y la Policía Nacional una calificación promedio de 3,74 , en tanto que, el Instituto Ecuatoriano de Seguridad Social es la institución que menor calificación obtuvo en promedio de la ciudadanía con el 2,98\%. 


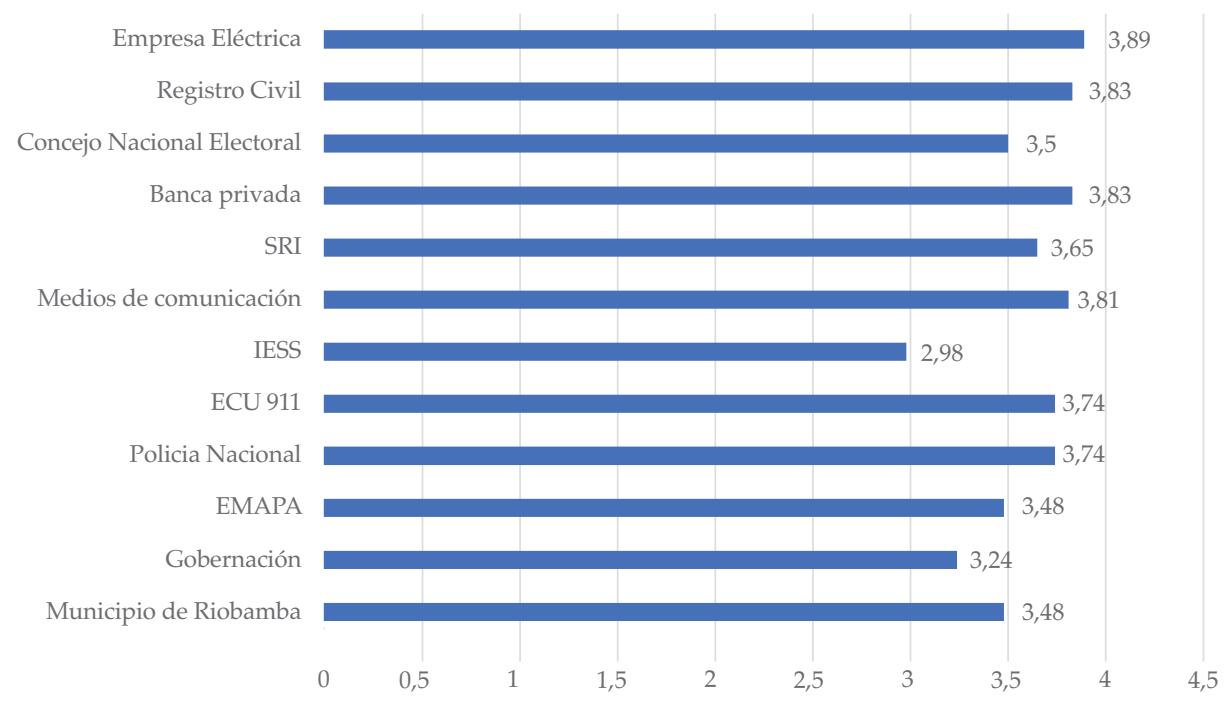

Figura 7. Imagen de las instituciones de la ciudad de Riobamba

Fuente: elaboración propia con base en la encuesta de opinión pública aplicada en Riobamba. Abril 2018.

Nivel de credibilidad de las autoridades de la ciudad de Riobamba

En cuanto al nivel de credibilidad que los encuestados tienen acerca de las autoridades de la ciudad de Riobamba, se pudo establecer lo siguiente: el Alcalde de la ciudad de Riobamba y el-Prefecto de la provincia de Chimborazo obtuvieron el más alto nivel de credibilidad con 2,66 respectivamente, seguidos en credibilidad por la Gobernadora de la provincia con 2,60, y con el peor nivel de credibilidad están los concejales de Riobamba y los asambleístas que representan a la provincia de Chimborazo con un 2,28 y 2,22 en promedio respectivamente. 


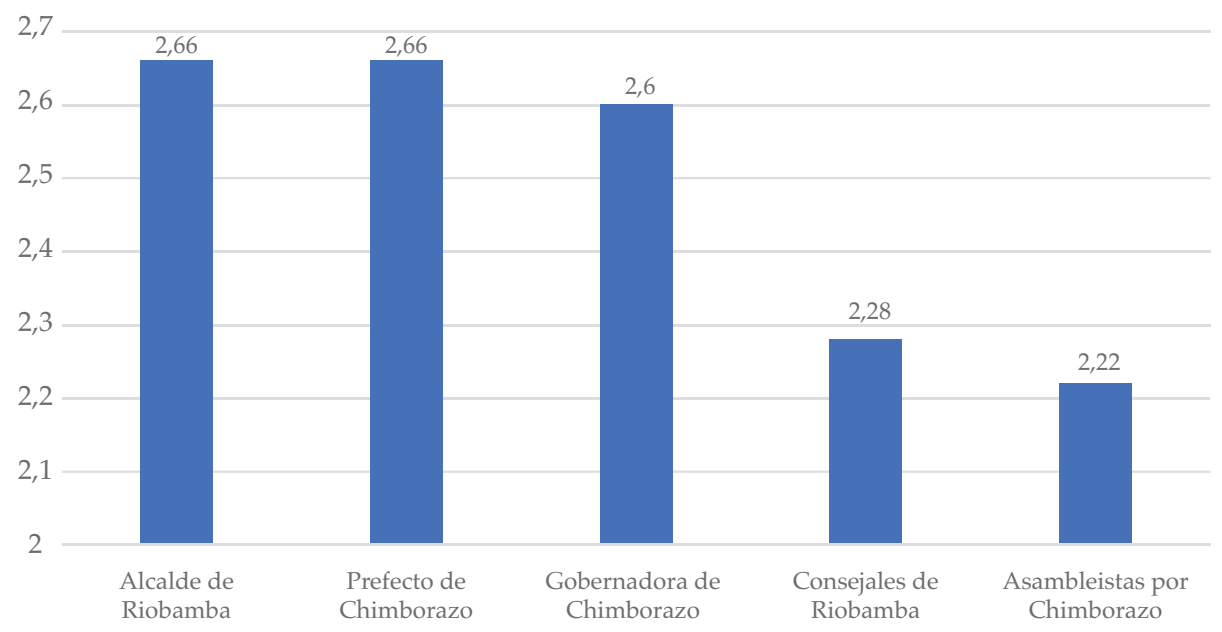

Figura 8. Nivel de credibilidad de las autoridades de Riobamba

Fuente: elaboración propia con base en la encuesta de opinión pública aplicada en Riobamba. Abril 2018.

\section{Opinión sobre la calidad de los servicios}

La ciudadanía de Riobamba también se manifestó en cuanto a los diferentes servicios prestados por las diferentes instituciones en donde se pudo determinar que el servicio de alumbrado público que presta la Empresa Eléctrica Riobamba tiene la evaluación más alta en la calificación, con un promedio de 3,91, seguido por el servicio de suministro del agua potable que lo realiza la Empresa Pública de Agua Potable del GAD de Riobamba, con el 3,88 en promedio. El servicio de mantenimiento de vías y calles es la prestación que menos calificación adquirió en la encuesta con el 3,18 . 


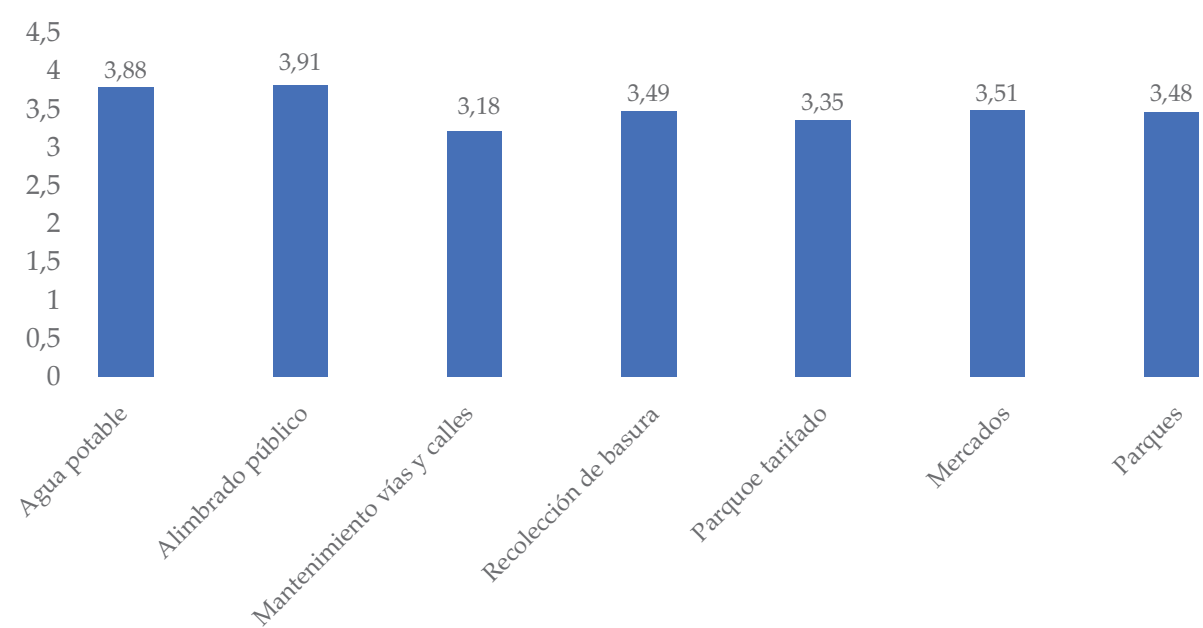

Figura 9. Encuesta sobre la calidad de los servicios públicos

Fuente: elaboración propia con base en la encuesta de opinión pública aplicada en Riobamba. Abril 2018.

\section{Instituciones de educación superior}

Por la declaración de Riobamba como ciudad universitaria, se preguntó a la ciudadanía su opinión acerca de la función, credibilidad, confiabilidad de las dos instituciones de educación superior más importantes de la ciudad de Riobamba y la provincia de Chimborazo, a la que la ciudadanía respondió lo siguiente: la credibilidad que tiene la ciudadanía de la Escuela Superior Politécnica de Chimborazo (Espoch) es muy alta con el 4,95\%, mucha credibilidad con el $15,10 \%$, algo de credibilidad con el $36,72 \%$, poca credibilidad con el $29,17 \%$ y nada de credibilidad con el $14,06 \%$, en tanto que, la Universidad Nacional de Chimborazo (Unach) la credibilidad que tiene la ciudadanía de esta institución de educación superior es muy alta con el 1,57\%, mucha credibilidad con el 9,16\%, algo de credibilidad con el 30,10\%, poca credibilidad con el 29,32\% y nada de credibilidad con el $29,84 \%$. Sin embargo, estimando estadísticamente los promedios el nivel de credibilidad de las dos instituciones obtienen un nivel de 
credibilidad que oscila entre buena y muy buena, es decir entre 4,37 y 4,21 respectivamente.
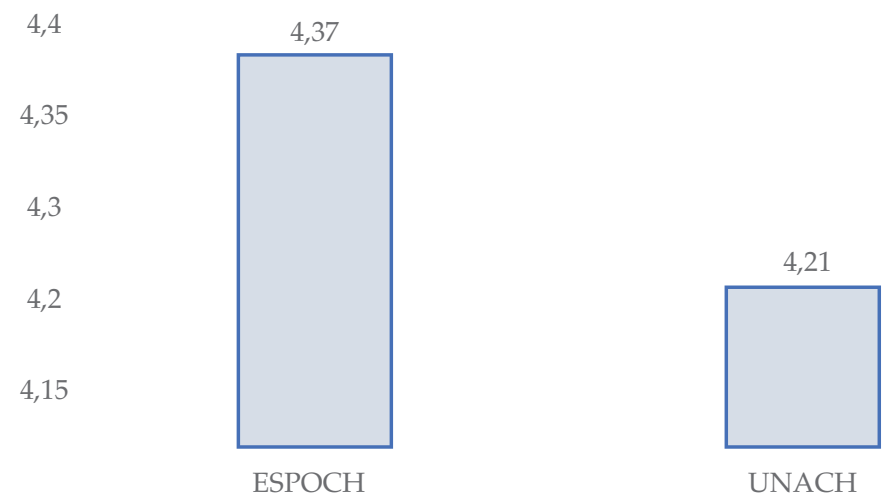

Figura 10. Imagen de las instituciones de educación superior de Riobamba Fuente: elaboración propia con base en la encuesta de opinión pública aplicada en Riobamba. Abril 2018.

\section{Instituciones productivas más representativas}

En cuanto a la imagen que tienen los encuestados de ciertas instituciones productivas de la ciudad de Riobamba, mediante una comparación de las medias se obtuvo los siguientes datos: la empresa Cemento Chimborazo y la empresa Tubasec, según las encuestas, cuentan con la mejor imagen con 3,78 y 3,77 , en tanto que la percepción que tienen de la imagen, desempeño y productividad del Parque Industrial es regular, ubicándose en un promedio de 3,0. 


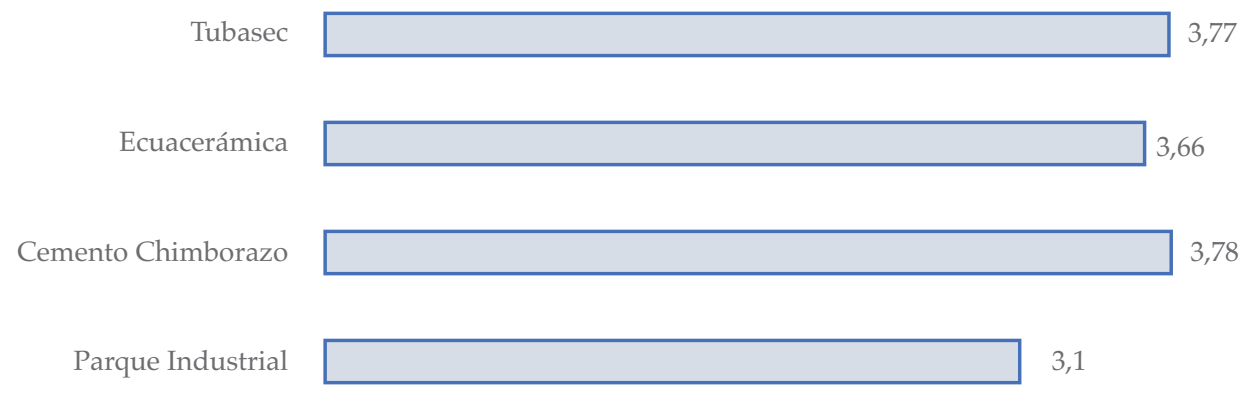

Figura 11. Imagen de las instituciones de producción de Riobamba Fuente: elaboración propia con base en la encuesta de opinión pública aplicada en Riobamba. Abril 2018.

\section{Aspectos locales más relevantes}

\section{Transporte}

El servicio de transporte, que incluye el de buses y tde axis en la ciudad, según los datos obtenidos en la encuesta de opinión, catalogan a la calidad del servicio de buses como regular, con un promedio estadístico de 2,99 sobre cinco, considerando que la calificación está entre mala y regular, se puede evidenciar varias falencias y problemas que no le permiten brindar un buen servicio. En cuanto al servicio de taxis tiene una media de 3,34 sobre cinco, lo cual demuestra que su calidad del servicio es regular, tomando en cuenta que su calificación no es tan baja, pero que aún requiere ciertos ajustes en lo referente al servicio que deben brindar. 


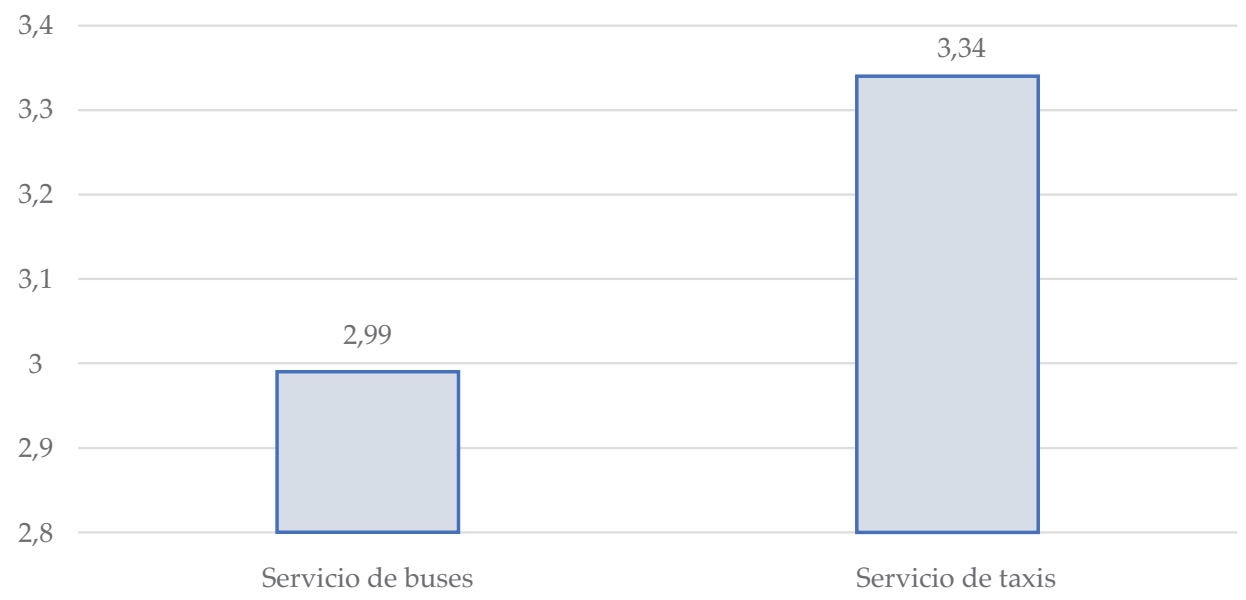

Figura 12. Calidad del servicio de transporte en buses y taxis Fuente: elaboración propia con base en la encuesta de opinión pública aplicada en Riobamba. Abril 2018.

Intención de voto de los posibles candidatos para la alcaldía de Riobamba 2019

Al indagar a los ciudadanos, si a la fecha de la elaboración de la encuesta, los ciudadanos ya tenían definido un candidato para Alcalde de Riobamba, los ciudadanos respondieron de la siguiente manera: el 88,54\% afirmaron que no tenían aún definido un candidato, el 5,21\% se inclinaron por Napoleón Cadena, el 1,82\% por José Luis Díaz, el 1,04\% para Edmundo Sancho, otro 1,04\% para Paúl Mancero, el 0,78\% votarían por Mariano Curicama, y por último un $0,26 \%$ para cada uno de los siguientes candidatos: Pedro Curichumbi, Luis Carvajal, Juan Pablo Cruz, Evaristo Torres y Ángel Yánez. 


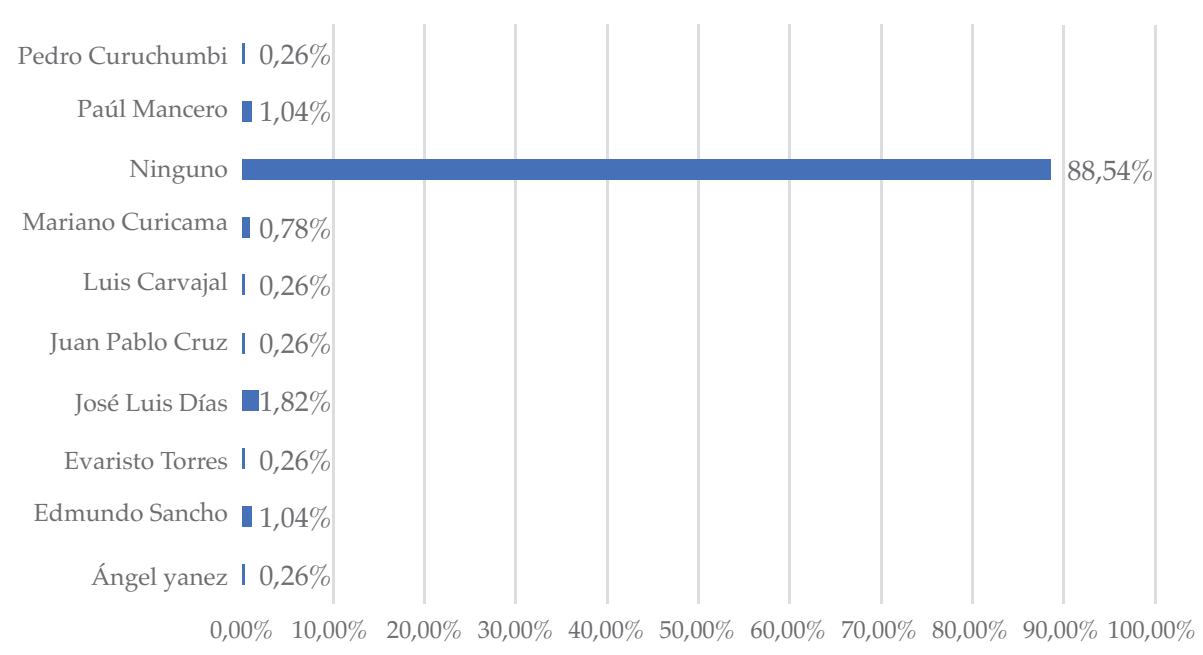

Figura 13. Intención de voto de los posibles candidatos para la alcaldía de Riobamba 2019

Fuente: elaboración propia con base en la encuesta de opinión pública aplicada en Riobamba. Abril 2018.

\section{Seguridad ciudadana}

Se le consultó también a la ciudadanía acerca de, si alguna vez fue víctima de actos delictivos en la ciudad, a lo cual respondieron: el $22 \%$ ha sido afectado por robo a sus viviendas, el $18 \%$ fue víctima de robo y asalto, el 15\% tuvo algún tipo de amenaza, el 12\% fue víctima de robo de su vehículo y el $9 \%$ fue víctima de estafa. Sin embargo y pese a la existencia de estas cifras, la ciudad de Riobamba es considerada una de las ciudades más seguras a nivel nacional. 


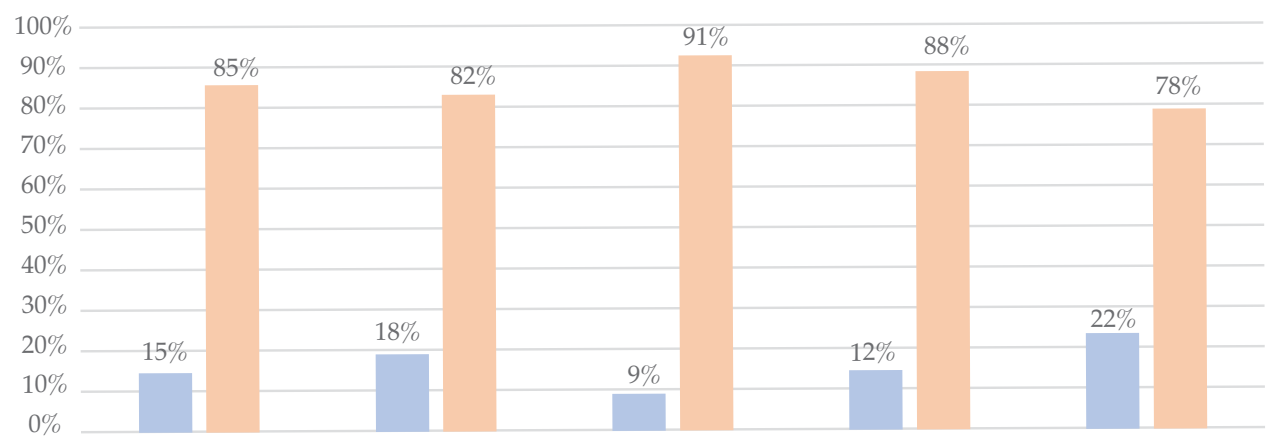

Figura 14. Percepción de la seguridad ciudadana en Riobamba

Fuente: elaboración propia con base en la encuesta de opinión pública aplicada en Riobamba. Abril 2018. 


\section{Conclusiones}

La rigurosidad que implican los estudios de las percepciones en la sociedad de aspectos de interés público y privado demanda de procesos metodológicos y científicos comprobados. Las encuestas de opinión pública toman importancia a través del rol que cumplen las universidades, debido a varios factores como la imparcialidad y confidencialidad de la información. A pesar de la crítica de diversos autores, las encuestas de opinión resultan ser un instrumento susceptible de aplicación, siempre y cuando se realicen de forma periódica para un pronóstico acertado.

La ciudad de Riobamba cuenta con diversos servicios públicos como son: alumbrado público que presta la Empresa Eléctrica, este tiene la evaluación más alta con un promedio de 3,91, seguido por el servicio de suministro del agua potable que lo realiza la Empresa Pública de Agua Potable y Alcantarillado con el 3,88 en promedio, por lo que en relación con la escala de Likert se calificaría como un servicio bueno; y finalmente el servicio de mantenimiento de vías y calles tiene una calificación promedio de 3,18 siendo la que menor nivel de satisfacción genera, por lo que se calificaría como un servicio regular.

La ciudadanía del cantón Riobamba tiene una percepción definida de la gestión de sus autoridades e instituciones, con una percepción de mala y regular a su mayoría de entidades, exceptuando a las entidades de educación superior como la Escuela Superior Politécnica de Chimborazo y la Universidad Nacional de Chimborazo, las mismas que tienen una muy buena imagen frente a la ciudadanía, pese a ser entidades educativas jóvenes, especialmente la Universidad Nacional de Chimborazo (Unach). 


\section{Referencias}

Boladeras, M. (2001). La opinión pública en Habermas. Anàlisi: quaderns de comunicació i cultura, (26), 51-70.

Bourdieu, P. (1992). La Opinión Pública no existe. Debates en Sociología, (17), 302-311.

Castro, L. (2012, 23 de febrero). Psicoloquio. http://psicoloquio. net / la- importancia-de-la-encuesta-de-opinion-publica /

Cuesta, J. A. (1984). Medidas de centralización multidimensionales (ley fuerte de los grandes números). Trabajos de Estadística y de Investigación Operativa, 35(1), 3-16.

Dewey, J. (1927). The public and its problems. New York: Holt, Rinehart y Winston.

Ginsberg, B. (1986). The Captive public: How mass opinion promotes state power. Nueva York: Basic Books.

Guitiérrez, L. (2017, 07 de marzo). El Tiempo. Opinión pública en la sociedad de la red. Obtenido de: https: / / www.eltiempo. com.ec/noticias / columnistas / 1 / opinion-publica-en-lasociedad-de-la-red

Huneeus, C. (2010). Las encuestas de opinión pública en las nuevas democracias de América Latina. La sociología en sus escenarios (19), 9-30.

INEC. (2010). Resultados del Censo 2010 de la población y vivienda. Fascículo provincial de Chimborazo. Obtenido de: https:// www.ecuadorencifras.gob.ec/wp-content/descargas / Manu-lateral / Resultados-provinciales / chimborazo.pdf

Lazarfeld, P., Katz, E., \& Roper, E. (2017). Personal influence: The part played by people in the flow of mass communications. Emek: Routledge.

Likert, R. (1932). A technique for the measurement of attitudes. Archives of Psychology, 140, 55.

Lippmann, W. (1995). Public Opinion. New York: Harcourt Brace Jovanovich.

Martín-Baró, I. (1998). El papel desenmascarador del psicólogo. Psicología de la liberación, 161-199. 
Mora, M. (2005). El poder de la conversación. Elementos para una teoría de la opinión pública. Buenos Aires: La Crujía Ediciones.

Noelle-Neumann. (1995). Hacia una teoría de la opinión pública. En: La espiral del silencio. Barcelona: Paidós Comunicación.

Ojeda, S., Gontero, S., y Ceballos, N. (2010). La verdad de las encuestas de opinión pública. La Sociología en sus Escenarios, 1-18.

Pareja, N., y Echeverría, M. (2014). La opinión pública en la era de la información. Propuesta teórico-metodológica para su análisis en México. Revista mexicana de opinión pública(17), 51-68. https: / / doi.org/10.1016/s1870-7300(14)70899-3

Raig, J. (1999). Opinión pública y comunicación política en la transición democrática. 1-900. Universidad de Alicante. Obtenido de: http://www.cervantesvirtual.com/ descargaPdf/opinion-publica-y-comunicacion-politicaen-la-transicion-democratica--0/

Tocqueville, A. (1835). La democracia en América (D. S. Aleu, Trad.). Madrid: Editorial Trotta.

WAPOR. (2018). World Association for Public Opinion Research. Obtenido de Freedom to Conduct Opinion Polls a 2017 Worldwide Update. Obtenido de: https://wapor.org/ wp-content/uploads / ESOMA-WAPOR_Freedom-toConduct-Opinion-Polls-Final-incl-edits.pdf 


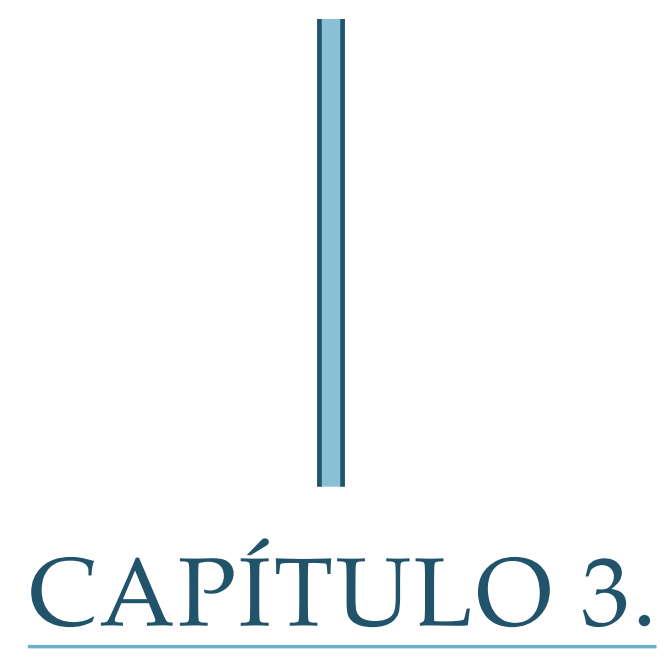

\section{THE ECUADORIAN FLOWER CLUSTER}

Daysi Astudillo-Condo Fausto Erazo- Guijarro Sandra Huilcapi-Peñafiel Pablo Ochoa-Ulloa 


\section{Abstract}

The research work aims to show the importance of the development of Clusters in the flower sector of Ecuador. Determine its species, characteristics, export conditions and expansion in the world market. The macroeconomic context explains factors such as: education, competitiveness, government participation, supply and demand conditions; The same ones that determine the construction of conglomerates considering the "Porter's Diamond" methodology. The analysis of the Porter Diamond in this specific study shows that Ecuador is an ideal country for planting roses, a relevant product within the different flower species, due to its geographical location, climatic diversity, soil conditions and other characteristics. with which it is possible to obtain a quality product. Ecuador over time, has managed to become more technical in the production process as part of its value chain. Several are the industries that have been involved in this production process, benefiting the different regions of the country, enhancing the productive matrix and boosting the market for goods and services, the labor market and the money market. Ecuador is also one of the main flowers exporting countries in the world. Several states located especially in North America, Europe, and Asia, show a preference for Ecuadorian roses due to their price and quality.

Key Words: cluster, flowers, value chain, competitive, industries. 


\section{Introduction}

Ecuador is in South America and has 276,841 square kilometers of land and 6,720 square kilometers of water, making it the 74th largest nation in the world with a total area of 283,561 square kilometers. In addition, sharing two borders with Colombia in the nort and Peru in the south. On the side of the western coast is the Pacific Ocean and has a volcanic section of the Andes which divides the territory from north to south. The currency of Ecuador is the US Dollar (adopted by the president Jamil Mahuad during the 2000 after a huge economic crisis). Ecuador has 16 million inhabitants, making it the eighth most populous country in Latin America.

The capital of Ecuador is Quito, its official language is Spanish which is spoken by $99 \%$ of the population, but also has 13 indigenous languages, including Kichwa and Shuar. Benefited by the number of rivers that circulate through it as well as being one of the most biodiverse countries in the world exporter of oil, bananas, flowers, shrimp and cacao. In addition, receiving many foreign tourists, which makes it a benchmark in regional tourism reception.

The flower industry dates to the 1980s and it started growing by 1991 when they signed the Andean Trade Preference Act (ATPA), which granted market access with zero tariffs for flowers. The country has excellent weather conditions that is why they produce flowers with a lot of varieties and characteristics that are highly valued in the international market.

Expoflores, is the main actor in the cluster map, representing the industry, they are making a great effort to increase the international demand and to stimulate the investment in some opportunity areas. 
Throughout this investigation we will focus in the roses subcluster, using several tools proposed by Michael Porter in order to identify Ecuador's value proposition, and giving recommendations in order to improve its competitiveness.

\section{The Ecuadorian world cluster status}

The world market of flowers is especially important, there are different countries that had develop clusters and subclusters around this industry. The flower trade today is a major industry in both developed and developing countries, whose estimated global value is more than $\$ 100$ billion a year (BBC News, 2016). Flowers have been exported around the world for different occasions, this gives a big opportunity of success because there is a big market. The world export market for flowers and flowerrelated products was almost $\$ 30$ billion in 2009 (Porter, Ramírez \& Van, 2013).

The consumption of flowers in the world is more common in countries where their growth is more difficult, and they see it as an exotic and luxury product. It is so, that in the wealthiest countries tend to consumption flowers in great quantity. In 2008, the average Dutch citizen bought $\$ 80$ worth of flowers per year, while the average American spent \$33. In Germany, the percentage of households that bought flowers was 76\% versus $63 \%$ in Great Britain, 60\% in France, and 28\% in the United States. Per capita consumption in China was estimated at less than $\$ 1$ (Porter, Ramírez \& Van, 2013).

Netherlands have been the most important country of the industry because of their investment, logistic and their auction program. They are the first exporter of flowers around the world. The second exporter is Colombia and is the direct competitor of 
Ecuador because they have almost the same natural conditions of growth (see Exhibit 3). The first importer of flowers in the world is United States and is the principal client of Ecuador, followed by Germany and United Kingdom (see Exhibit 4).

\section{Relevant cluster charts: flower industry status in Ecuador}

\section{Macroeconomic situation of Ecuador}

Based on data provided by the Association of Banks of Ecuador (Asobanca), the years 2015 and 2016 have recorded the lowest performances since the Ecuador adopted the dollar as its local currency. In 2015, growth was only $0.2 \%$ and for 2016 and based on data from the Economic Commission for Latin America and the Caribbean (CEPAL, 2016) there was a decrease of 2.5\%. According to data from the Central Bank of Ecuador (BCE), the years 2004, 2005, 2008, 2011, 2012 and 2013 were the highest growth rates, with rates exceeding $4.5 \%$ per year. The main growth promoter for 2004 and 2005 was private investment, especially for the implementation of the heavy crude oil pipeline. On the other hand, growth in 2008, 2011, 2012 and 2013 was the result of increased spending in the public sector that displaced private investment. (INEC, 2016). 


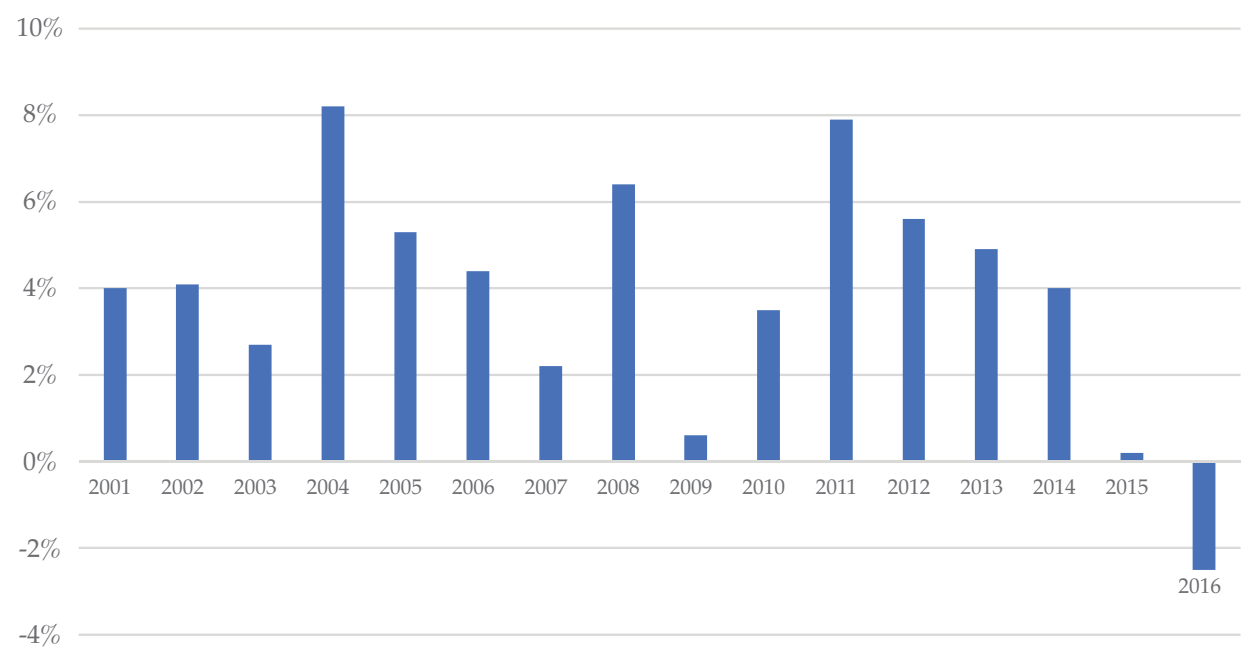

Figure 15. Annual economic growth

Source: Prepared by the authors based on data supplied by Central Bank of Ecuador (2018).

\section{Education in Ecuador}

Education in Ecuador is regulated by the Ministry of Education, divided into fiscal, fiscomisional, municipal and private education, secular and religious, Hispanic, or bilingual intercultural education. Public education is secular at all levels, compulsory and free until high school. The General Basic Education (E.G.B.) aims to develop the skills, abilities and competencies of children and adolescents from 5 years of age and up until continuing high school.

Ecuador has always been burdened with a poor level of education. This is very worrying for a society that is developing and can be seen in the current illiteracy rate of $5.8 \%$. The great problem of Ecuador in terms of education is a problem of the quality of spending, since it is not important to spend more, but to spend better. Based on data the Global Competitiveness Report of 
the World Economic Forum 2015-2016 Ecuador has made large investments in primary education infrastructure, surpassing the level of countries in Latin America and the Caribbean, as shown in the table:

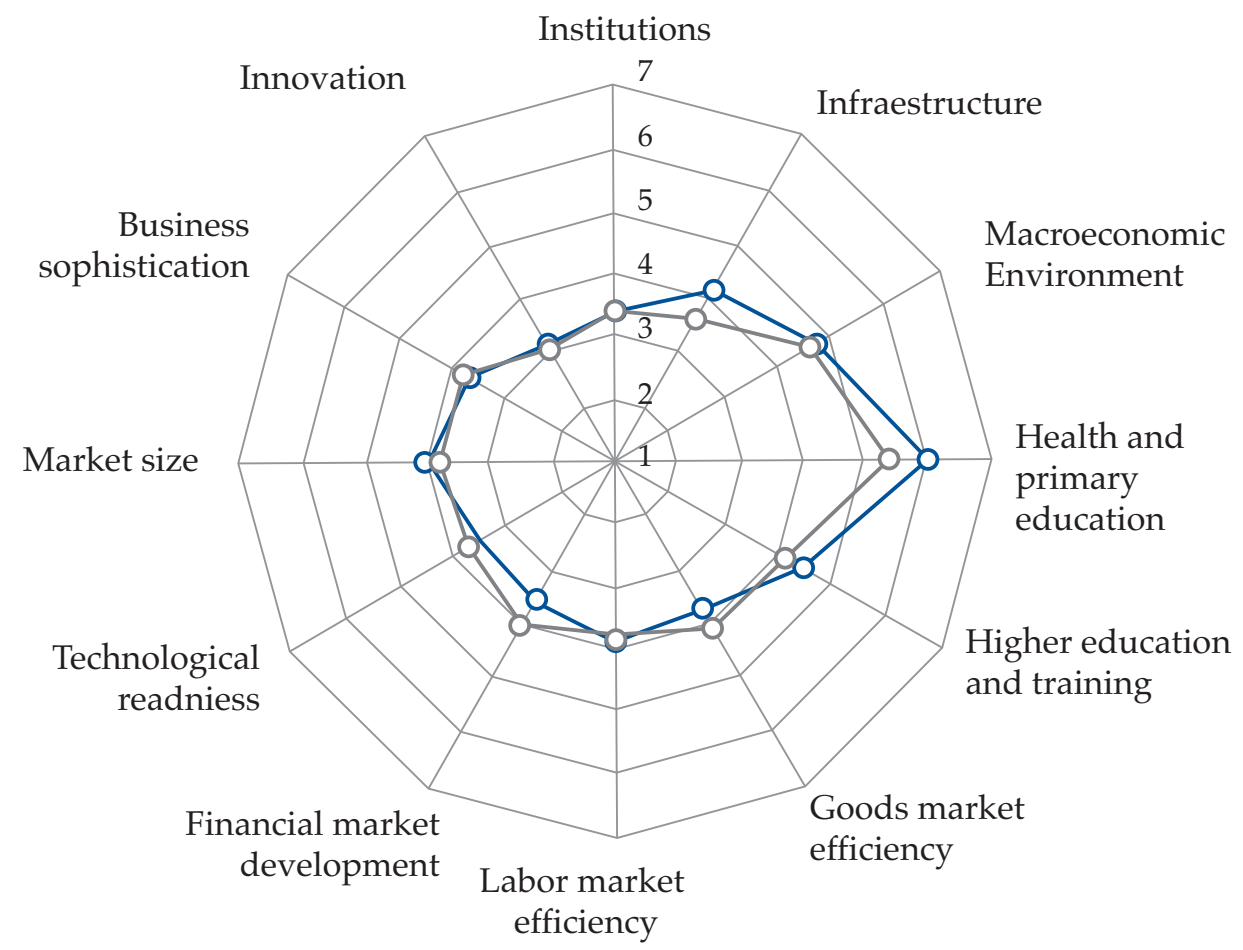

- - Ecuador - - L Latin America and the Caribbean

Figure 16. Global competitiveness report - Ecuador

Source: World Economic Forum (2016).

Several factors have undermined the country's competitiveness, lack of infrastructure as well as inadequate labor force education. 
The most problematic factors for doing business

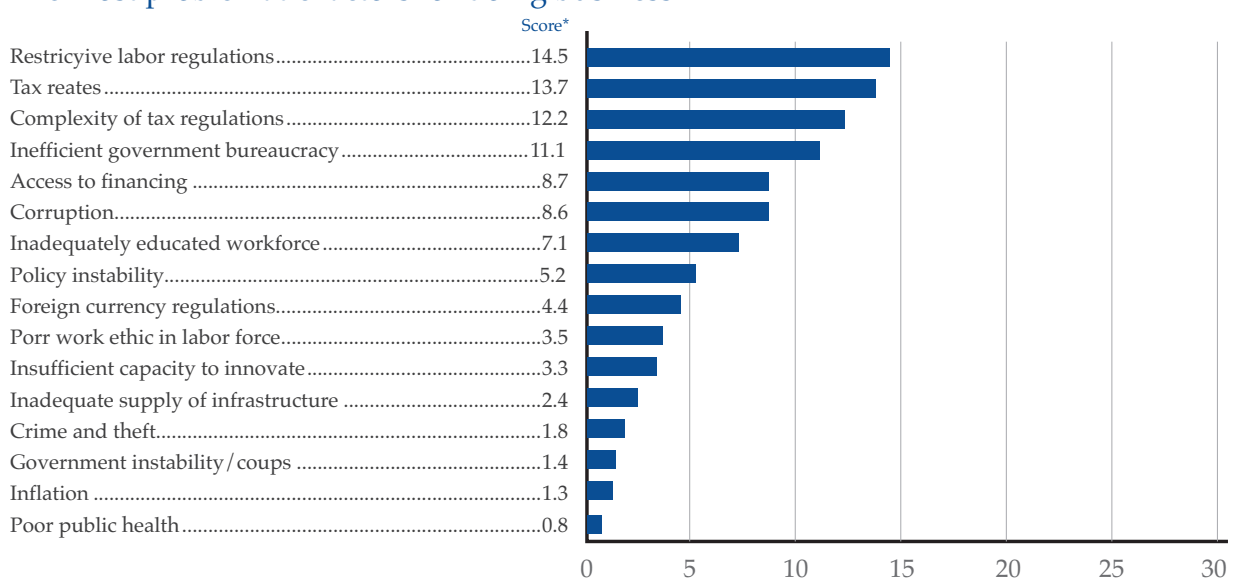

Figure 17. The most problematic factors for doing business

Source: World Economic Forum (2016).

\section{Ecuador: evolution of flowers exporting}

In 2016, the floriculture sector has experienced a slight drop in exports compared to 2015. This decrease is the result of the fall of several markets, especially the second most important for the country, the Russian market. Several factors negatively influenced the behavior of Ecuadorian exports. The appreciation of the dollar and the depreciation of the currencies of the main markets, such as the European and the Russian, strongly impacted the importers, who paid more for the same quantity of the product. In another aspect, the ease of devaluing the currency and lower production costs by the main competitors of the country Colombia, Kenya and Ethiopia, put the country at a disadvantage, because Equatorial flowers are traded at higher prices. 


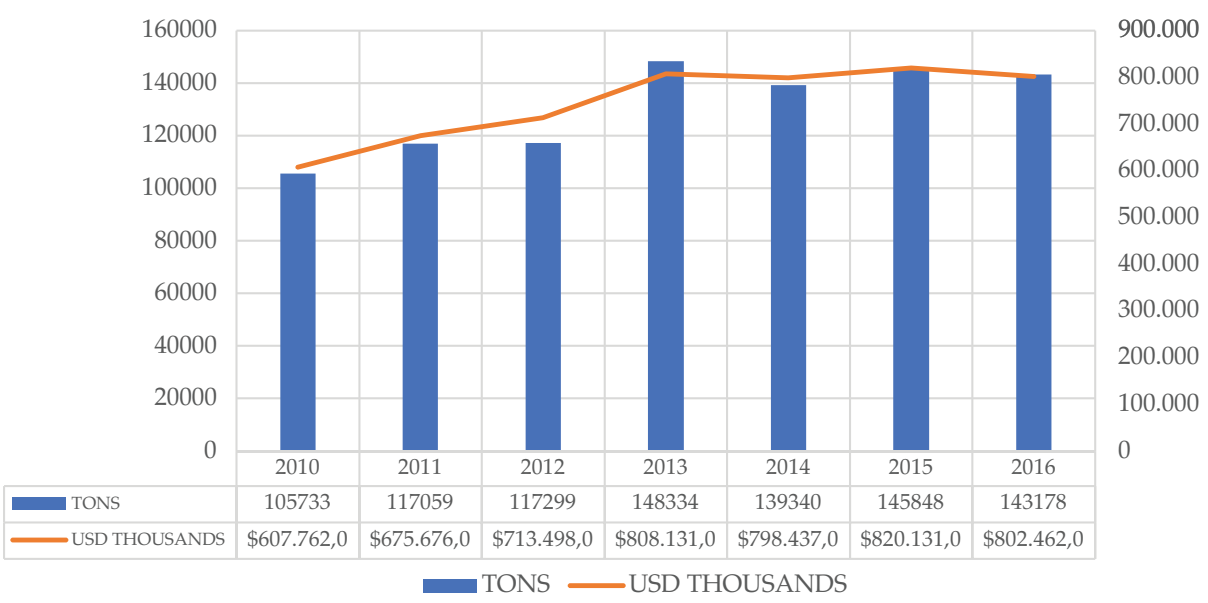

Figure 18. Total exports of flowers in Ecuador

Source: Prepared by the authors based on data supplied by Central Bank of Ecuador (2018).

In terms of volume, in 2016 exports totaled 143 thousand tons, showing a drop of $1.8 \%$ in relation to the year 2015 .

\section{Ecuador: total exports by type of flower}

Exports by type of flower maintain the trend that has been taking place in recent years, keeping as main product the shipments of roses $(75 \%)$, followed by other summer flowers $11 \%$, gypsophila with $8 \%$, carnations with $2 \%$, lilies with $1 \%$ and others with $3 \%$.

\section{Ecuador: market share}

In 2016, Ecuadorian flowers were exported mainly to the United States with a $48 \%$ share, making it the main trading partner since the beginning of floriculture. As in 2015, Russia stopped positioning itself as the second market for Equatorial flowers, since it registered a 1\% fall for the year 2016. In 2014 this market represented $22 \%$ of exports, falling $7 \%$ in 2015. Moreover, the 
countries that make up the European Union have obtained the second place of exports in 2016, presenting a decrease of $1 \%$.

\section{MARKET SHARE 2015}
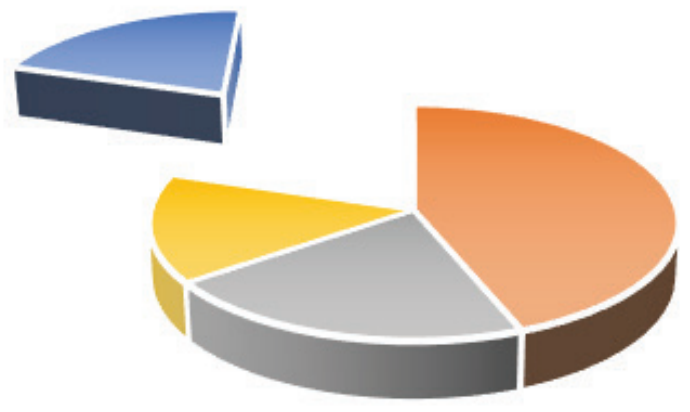

MARKET SHARE 2016

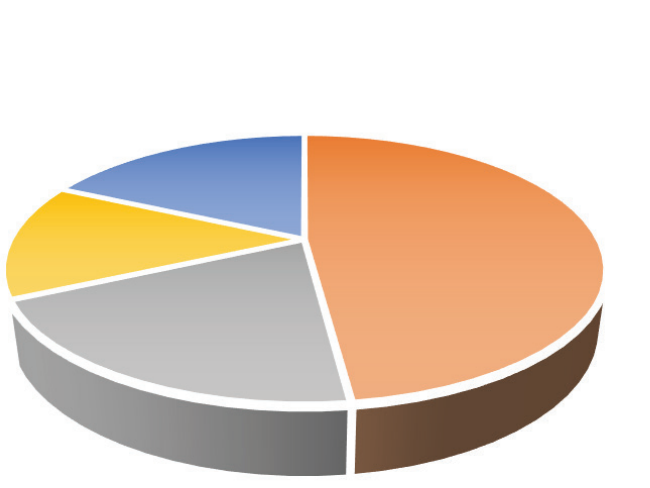

- MARKET SHARE 2015

- ESTADOS UNIDOS

UNION EUROPEA

- RUSIA
MARKET
SHARE 2016
ESTADOS UNIDOS
UNION EUROPEA

- RUSIA

- OTHERS

Figure 19. Market share 2015 - 2016

Source: Prepared by the authors based on data supplied by Central Bank of Ecuador (2018). 


\section{Prices by type of flowers}

According to Central Bank of Ecuador data there was a 0.8\% increase in the price of roses, which rose up from USD 5.43 in 2015 to USD 5.47 in 2016. In addition, a growth in the other varieties of flowers as detailed in the table below:

Table 10. Prices by type of flowers

\begin{tabular}{|c|c|c|}
\hline Description & Year & Price/USD \\
\hline \multirow{2}{*}{ Roses } & 2015 & 5.43 \\
\hline & 2016 & 5.47 \\
\hline \multirow{2}{*}{ Other flowers } & 2015 & 5.96 \\
\hline & 2016 & 5.85 \\
\hline \multirow{2}{*}{ Gypsophila } & 2015 & 6.55 \\
\hline & 2016 & 6.50 \\
\hline \multirow{2}{*}{ Carnations } & 2015 & 4.08 \\
\hline & 2016 & 4.19 \\
\hline \multirow{2}{*}{ Chrysanthemums } & 2015 & 8.95 \\
\hline & 2016 & 4.66 \\
\hline \multirow{2}{*}{ Lilies } & 2015 & 6.16 \\
\hline & 2016 & 6.45 \\
\hline \multirow{2}{*}{ Alstroemeria } & 2015 & 4.92 \\
\hline & 2016 & 3.84 \\
\hline \multirow{2}{*}{ Aster } & 2015 & 3.24 \\
\hline & 2016 & 3.90 \\
\hline \multirow{2}{*}{ Gerbera } & 2015 & 5.95 \\
\hline & 2016 & 5.16 \\
\hline \multirow{2}{*}{ Others } & 2015 & 14.94 \\
\hline & 2016 & 20.45 \\
\hline
\end{tabular}

Source: Prepared by the authors based on data supplied by Central Bank of Ecuador (2018). 


\section{Characteristics of the equatorian rose}

Currently in Ecuador there are 629 Floricultural estates registered in Agrocalidad, of which 471 plant roses. The flowers sector generates 105,000 direct and indirect jobs. An average of 12 people per hectare, of which $51 \%$ are women. Child labor has long been eliminated, so that legally constituted farms do not hire children. Ecuador is attractive because of its location; it has elevations on the equatorial line. The height at which roses are planted, goes between 2,600 and 3,000 meters, allowing for longer production cycles and with this, larger and wider stems and larger buttons.

\section{Geographical location of rose crops}

The province with the most crops is Pichincha where we find 75\% of the total hectares. Provinces like Cotopaxi own 19\%, Carchi and Imbabura contribute with $2 \%$ each, the rest of provinces represent $2 \%$. 


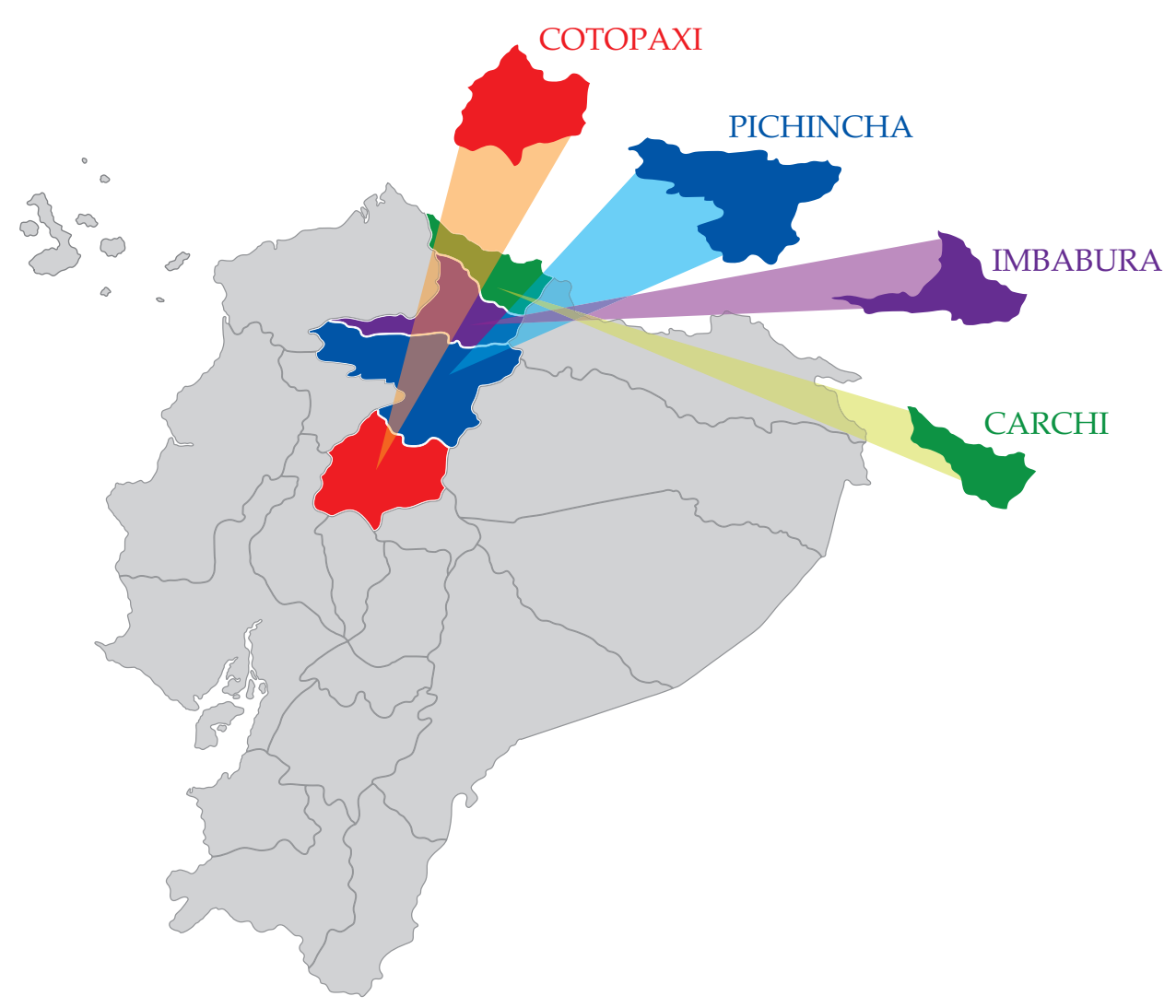

Figure 20. Geographical location of rose crops

Source: Prepared by the authors.

In Ecuador, the total number of flowers produced throughout the country, approximately 4,200 hectares, $74 \%$ correspond to roses.

\section{Exports of Roses}

By 2016, exports of roses fell by $0.7 \%$ in value and volume by $1.4 \%$ compared to 2015. 
Table 11. Exports of roses

\begin{tabular}{|c|c|c|c|c|}
\hline \multirow[b]{2}{*}{ Description } & \multicolumn{2}{|l|}{2015} & \multicolumn{2}{|l|}{2016} \\
\hline & Tons & USD thousands & Tons & $\begin{array}{c}\text { USD } \\
\text { thousands }\end{array}$ \\
\hline Roses & 11,445 & 604,657 & 109,855 & 600,570 \\
\hline $\begin{array}{l}\text { Other sommer } \\
\text { flowers }\end{array}$ & 14,607 & 87,075 & 15,493 & 90,569 \\
\hline Gypsophila & 10,036 & 65,767 & 9,436 & 61,367 \\
\hline Carnations & 4,21 & 17,189 & 3,562 & 14,942 \\
\hline Chrysanthemums & 988 & 8,845 & 1,058 & 4,929 \\
\hline Lilies & 1,183 & 7,284 & 992 & 6,397 \\
\hline Alstroemeria & 1,297 & 6,376 & 1,177 & 4,518 \\
\hline Aster & 318 & 1,029 & 283 & 1,106 \\
\hline Gerbera & 140 & 832 & 107 & 552 \\
\hline Azucenas & 330 & 1,644 & 493 & 2,603 \\
\hline Orchids & 0.44 & 89.4 & 1.08 & 13.9 \\
\hline Others & 1,295 & 19,343 & 728 & 14,896 \\
\hline Total & 145,848 & 820,131 & 143,187 & 802,461 \\
\hline
\end{tabular}

Source: Prepared by the authors based on data supplied by Central Bank of Ecuador (2018).

\section{Ecuadorian export destinations}

There are several destinations for Equatorial production. Among the main countries is the United States with a $41 \%$ share, followed by the European Union countries with $21 \%$, Russia with $18 \%$, Canada with $3 \%$, Ukraine with $2 \%$, and the rest of countries with 
a participation of $14 \%$, according to data from the Central Bank of Ecuador (BCE).

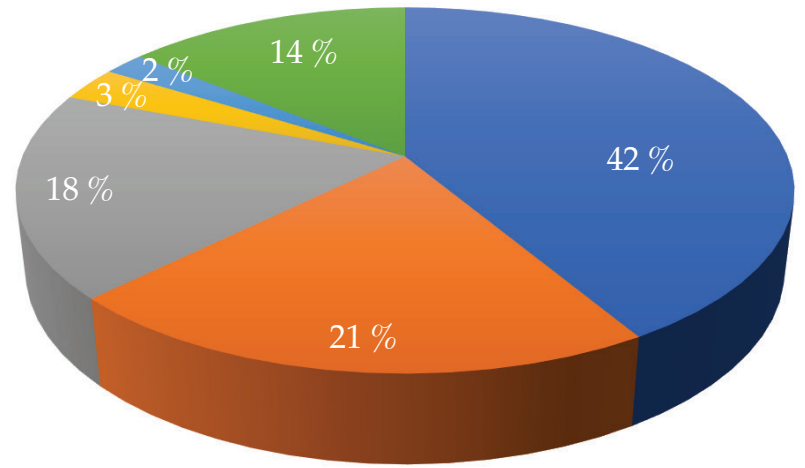

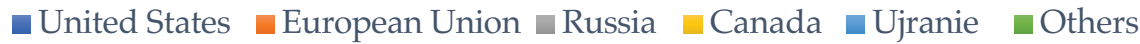

Figure 21. Export destinations

Source: Prepared by the authors.

\section{Competitive advantage of Ecuador as a supplier}

Pro Ecuador (2016, p. 9) mentions that the country is characterized by having a unique commercial strategy as floriculturists, this is a high number of producers and small farms that produce more than 400 varieties (more than twice the competitors). Producers seek to be at the forefront of the trends and fashion of the different markets. Internationally it become usual to produce few varieties on large areas of land, so that productivity would be the one that marked costs and competitive prices. The Equatorial farms are small and concentrate many varieties of flower. Today, competing countries are imitating this strategy as consumers are becoming more demanding.

In addition, several producers are entering the elaboration of preserved or eternalized flowers. 
Innovation, research, and development of the sector

Based on the data provided by PRO ECUADOR, in the country, there have been 10 years of research and development for new varieties through breeders, who carry out genetic crosses with Equatorial origin. Producing farms implement technification in the irrigation system, pest control, greenhouse temperature management, quality control and inventory management, so that they are increasingly more productive and have a lower percentage of flower rejection, which allows them to be more competitive. The Equatorial floricultural grows more and more in the elaboration of eternal roses that are in great demand in the Asian continent.

\section{The cluster diamond}

\section{Cluster Diamond Analysis}

The cluster diamond analysis of roses in Ecuador is presented in the next figure. 


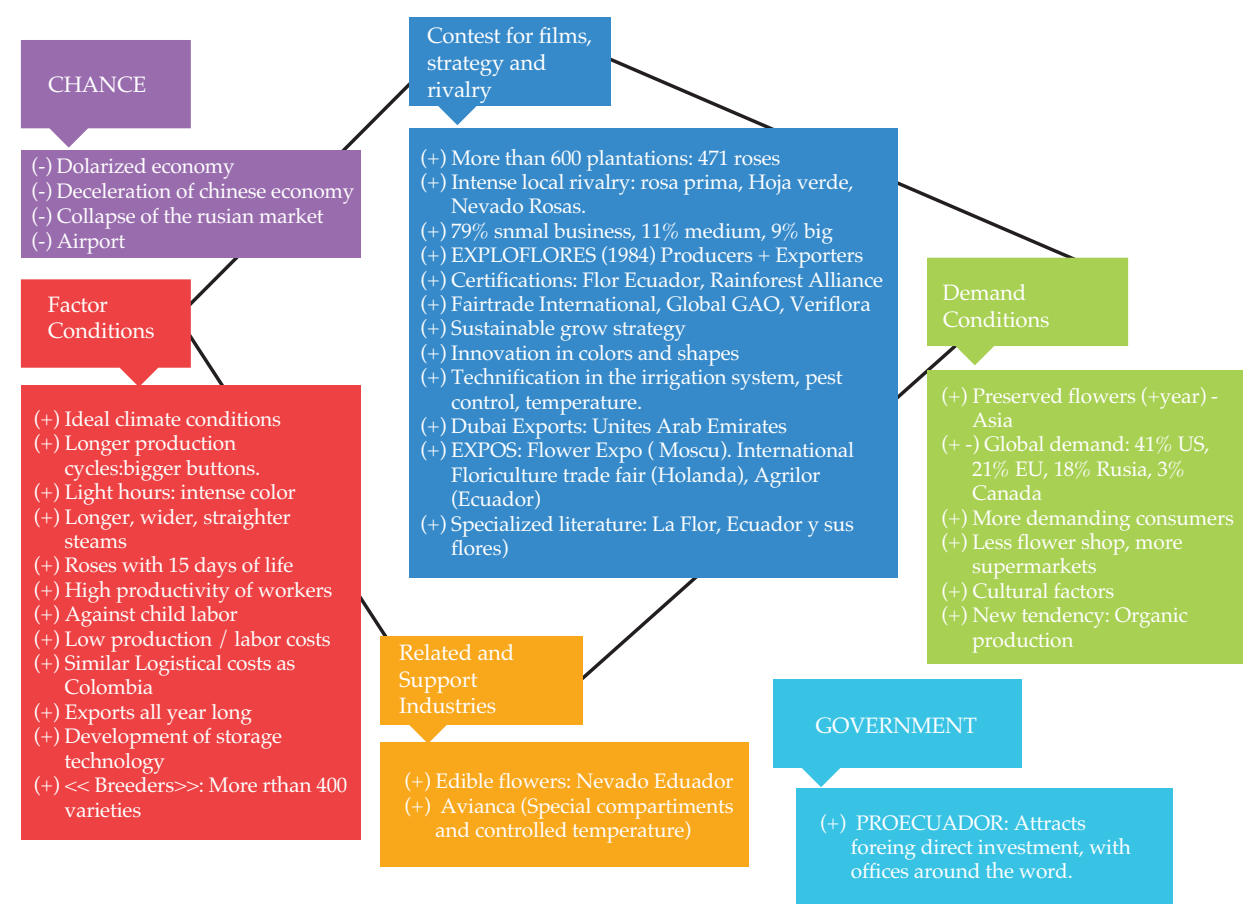

Figure 22. The cluster diamond analysis of roses in Ecuador Source: Prepared by the authors based on Porter (2008).

\section{Factor conditions}

According to Pro Ecuador (2016, p. 10)

Ecuador is an ideal country for the plantation of roses because of its ideal climate conditions which generate flowers with longer, wider, straighter stems and bigger buttons.

- Longer production cycles because of the ideal temperature.

- Ideal sunlight, having an impact on the intense color of the roses.

- Most of the companies are small (79\%), 11\% medium and 9\% small.

- $\quad 471$ rose plantations. 
- The plantation of roses generates 50,000 employments.

- Ecuador is against child labor.

- Specialized breeding industry, which creates rose varieties.

- Development of storage technology.

\section{Context for Firms Strategy and Rivalry}

According to Pro Ecuador (2016, p. 12):

In the last 10 years, Ecuador has special labs to develop new varieties and to improve irrigation systems, pest control, management of temperature in greenhouses and quality control.

\section{Specialized literature: La flor, Ecuador y sus flores}

Ecuador counts on different organizations, expos and certifications that improve the sub cluster's productivity.

\begin{tabular}{|l|l|}
\hline IFCs / Associations \\
\hline
\end{tabular}




\begin{tabular}{|c|c|}
\hline Dubai Exports & $\begin{array}{l}\text { Seeking to further explore business prospects } \\
\text { and export opportunities in the food and } \\
\text { beverage sector in Ecuador, PRO ECUADOR, the } \\
\text { trade and Investment promotion organization of } \\
\text { Ecuador and Dubai Exports, an agency of the } \\
\text { Dubai Department of Economic Development } \\
\text { (DED), organized a trade forum attended by } \\
\text { more than } 50 \text { local UAE companies. }\end{array}$ \\
\hline $\begin{array}{l}\text { AGRIFLOR } 2016 \\
\text { ocroBER } \\
5-7 \text { ECUAIO } \\
\text { QUDOR }\end{array}$ & $\begin{array}{l}\text { Expo: The largest in Latin America. Every two } \\
\text { years it has the goal to show the world the } \\
\text { competitiveness of the Ecuadorian flowers. }\end{array}$ \\
\hline & $\begin{array}{l}\text { International exhibition of Floriculture and } \\
\text { Green Industry (Russia). }\end{array}$ \\
\hline $\begin{array}{l}\text { Royal } \\
\text { Flora } \\
\text { Holland }\end{array}$ & $\begin{array}{l}\text { The cooperative must be the connector in the } \\
\text { horticultural sector and support the growth of } \\
\text { our customers and members. }\end{array}$ \\
\hline & $\begin{array}{l}\text { VDNH (Moscow) International Exhibition of } \\
\text { flowers, plants, equipment and materials for } \\
\text { ornamental gardening and flower business. }\end{array}$ \\
\hline$\frac{\substack{\text { SOCIETY of AMERICAN FLoRISTS } \\
\text { Your Growth is Our Business }}}{(1)}$ & $\begin{array}{l}\text { The Society of American Florists is the national } \\
\text { trade organization for all participants of the U.S. } \\
\text { floral industry and offers exciting benefits. }\end{array}$ \\
\hline
\end{tabular}

Source: Prepared by the authors based on Porter (2008). 


\section{Demand conditions}

According to Pro Ecuador (2016, p. 6):

- Consumers are demanding high quality roses, which Ecuador is able to produce.

- Consumers are starting to demand preserved flowers, which are made from fresh roses and can endure more than a year in perfect conditions.

- The United States recieves $41 \%$ of the production, EU $21 \%$, Rusia 18\%, Canadá, 3\%, Ucrania 2\% and the rest of the world $14 \%$.

According to BI. Directorate of Intelligence and Investments. (2016):

The demand condition of each country is due to cultural factors. For instance, Russia rather flowers with big and open buttons and more than $90 \mathrm{~cm}$ stems. In Central Europe the consumers rather bouquets with intense pinks and oranges, and in Asia they look for specific varieties of roses.

Consumers are looking for flowers that follow organic processes and fair trade.

\section{$\underline{\text { Related and support industries }}$}

According to BI. Directorate of Intelligence and Investments. (2016):

- Edible flowers are the last trend in the USA, demanding new standards and cultivation process. 
- Avianca Airlines, which transported 9620 tons of Ecuadorian flowers just for Valentine's day in 2016.

\section{Chance}

- Dollarized economy that affects the final price of the roses.

- Deceleration of Chinese Economy

- Collapse of the Russian Market

\section{Government}

PROECUADOR: attracts foreign direct investment, with offices around the world. It is the Institute for the Promotion of Exports and Investments, part of the Ministry of Foreign Trade, in charge of implementing the country's export promotion and investment policies and regulations, to promote the supply of traditional and non-traditional products.

\section{Value chain}

The value chain in the case of the roses of Ecuador is reflected in the complete process of sowing until the harvest and sales of the flowers. In this case, people are involved, especially women who plant and harvest the flower, to the facilities provided by the government so that the export of the flowers is the most appropriate for them to arrive at their destination fresh.

Being a perishable product, which has been treated to have a longer life than many other flowers, its value consists precisely in "the immortality of the rose" so that the flower growers and researchers work not only in the flower itself, but on the impact of everything that concerns it so that it reaches its destination as if it were still in its natural state. 


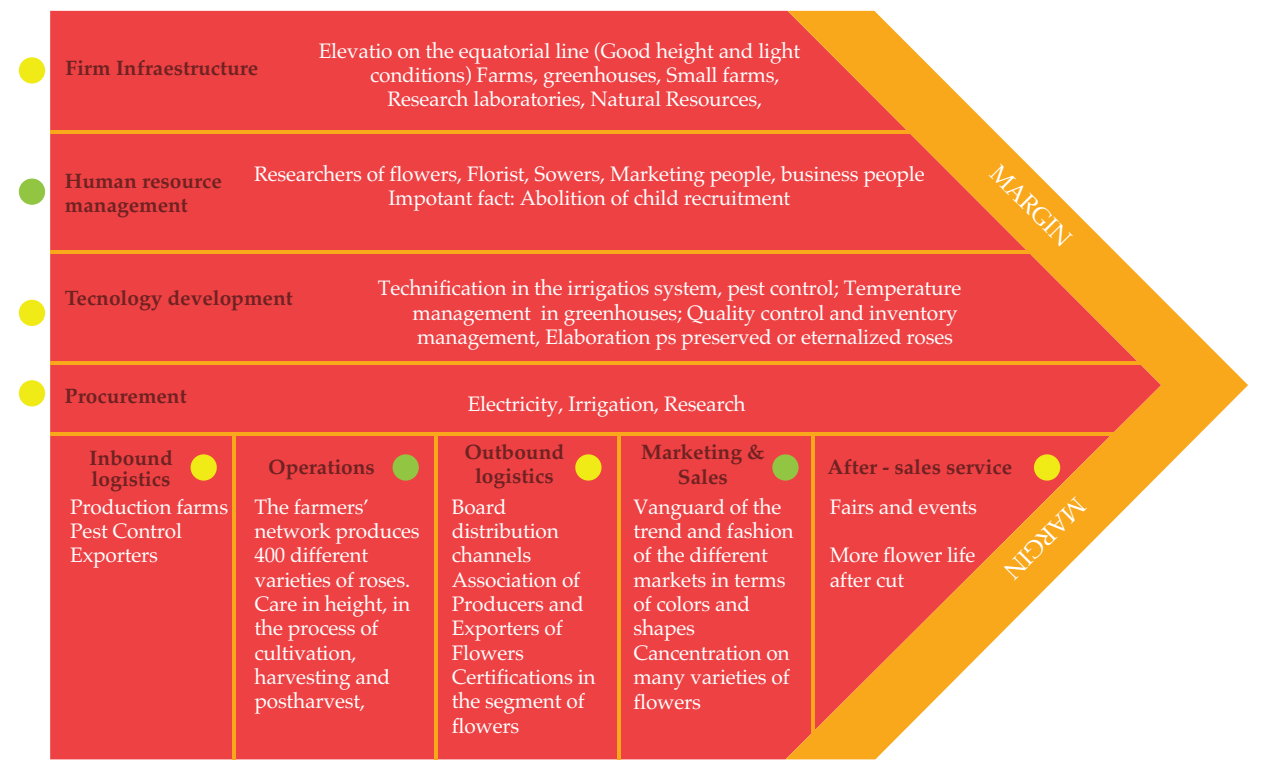

Figure 23. The value chain in the case of the roses of Ecuador

Source: Prepared by the authors based on Porter (2008).

The activities of support that affect the production of the roses are the following:

\section{Firm infrastructure}

The main element that has as an advantage Ecuador is its geographical position, being in the center of the Earth the conditions of light, elevation, and climate favor it in such a way that the flowers are affected in a positive way and reflect on the size of the stem and the head of the rose itself. In addition to the excellent climatic conditions the factors that directly intervene in them are the plantations, the greenhouses, the small farms and the research laboratories. 


\section{$\underline{\text { Human resources management }}$}

Ecuador employs many people for planting and collecting roses, one of the main features is that more than half of the people engaged in this activity are women, in addition to being found Very proud to have abolished the recruitment of children for these activities (Pro Ecuador, 2016, p. 8):

\section{Technology development}

The work that has been developed in this field is mainly in the case of people who are dedicated to research, especially working in the life of the flower, the least impact of pesticides on the environment and in the tones and grandeur of the rose itself. Adaptations have been made to the greenhouses so that these take full advantage of the climate conditions. The irrigation system allows to control and optimize the exact amount of water and fertilizers that should be applied to the flowers.

\section{Procurement}

The activities that are related to the purchasing process involved in the production of roses are basic services such as water, electricity, and gas, as well as the supply of soil, seeds and gardening equipment for planting. Ecuador not only takes advantage of the physical and preferential characteristics with which it is in the world and that allow you to have that type of roses, also require investing in research topics to Pro Ecuador (2016, p. 10): In the case of primary activities are those that are related to the production and marketing of roses and are: 


\section{Inbound logistics}

The most important in this case are the farms producing the flowers, which affect the yield of the cultivated hectare that goes from the producing farm, the number of plants that are planted per hectare, the variety of roses, the climatic conditions, the Height where it is cultivated, which in turn generates the control of irrigation, pest and maintenance of the roses until they are harvested, selected, packaged and translated to their different destinations to (Pro Ecuador, 2016, p. 12). Again, in this part intervene several distribution channels, whether by land or air.

\section{Operations}

One of the strengths is the process of growing roses, which Ecuador has well dominated, its harvesting and research system has allowed it to have more than 400 varieties of roses in the whole range of colors. Among the main characteristics is the care of the process of cultivation, harvest, and post-harvest. In addition to the physical characteristics of the flowers they are the color intensity, large buttons, perpendicularity and thickness of the stems, suitable texture and a life span of 15 days in the vase to Pro Ecuador (2016, p. 12).

\section{Outbound logistics}

For the flowers of Ecuador are particularly important the different certifications and that affect directly in their processes, reason why they have been installed laboratories of investigation and development of new own varieties by means of companies called "breeders" who are in charge to make genetic crosses with the native ones Ecuadorians. In addition, the farms seek to implement 
technologies in irrigation systems, pest control, temperature control, quality control and inventory management. In the same way they are subject to different certifications, the companies that can be mentioned are: Certified Florist ${ }^{\circledR}$, Rainforest Alliance ${ }^{\mathrm{TM}}$, Fair Trade ${ }^{\circledR}$ International, Global G.A.P., Veriflora ${ }^{\circledR}$ Certified, Organic Certified to Pro Ecuador (2016, p. 12).

\section{$\underline{\text { Marketing and sales }}$}

In addition to being distinguished by the variety and coloration of roses, the market has been looking for preserved roses, which are obtained from fresh roses and have at least one year of life. In addition to the traditional and seasonal sales made and the United States being one of its most important buyers to (Pro Ecuador, 2016, p. 11) at the time this research was carried out, the premiere of the Disney film Beauty and the Beast (El Comercio, 2017).

In addition to the quality tests and certifiers, Ecuadorian roses participate in different international fairs and events such as World Flora Expo (USA), Flower Expo (Moscow), International Floriculture Trade Fair (Holland) and Agriflor (Ecuador). 


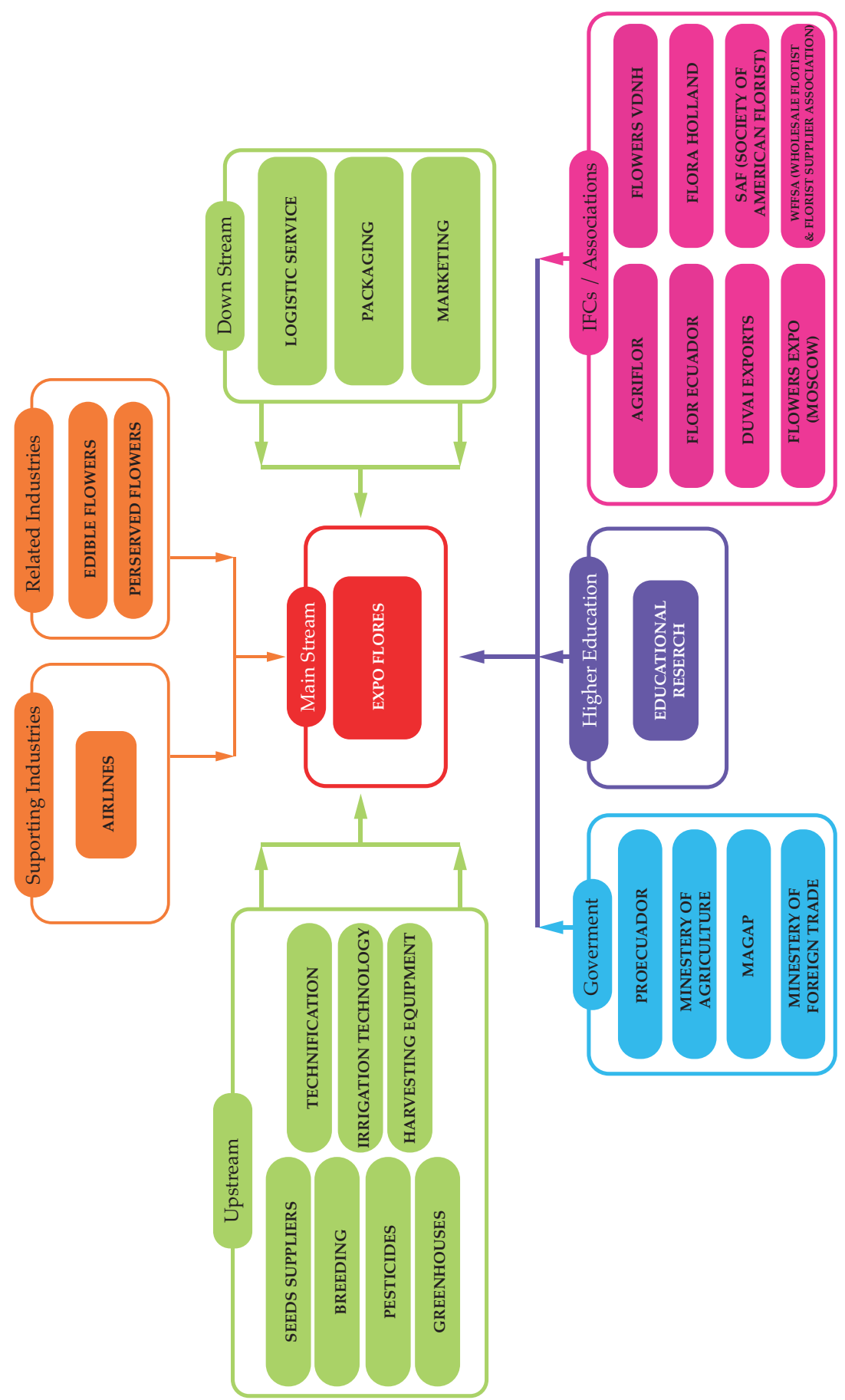

Figure 24. The value chain in the case of the roses of Ecuador

Source: Prepared by the authors based on Porter (2008). 


\section{Upstream}

The upstream players are the ones providing seeds, pesticides, technology, and machinery to the rose's cluster. Throughout the investigation we identified this player:

\section{Seed suppliers}

Breeding: In the last 10 years Ecuador has specialized laboratories focused on the development of new varieties of flowers. The national producers cultivate the new varieties, which are protected by the Intellectual Property System in Ecuador, and then export them. To do this they must pay royalties to the breeders. According to data from the (IEPI Ecuadorian Institute of Intellectual Property, 2014), there are 232 varieties of roses registered on the List of Plant Varieties in force, is subject to the payment of royalties. While on the Public Domain Plant Variety List there are 419 types of roses, which can be grown without restrictions.

A breeder takes five to eight years to create a new variety of rose through a hybridization process costing ten thousands of dollars. Depending on the size of the breeder laboratory, biotechnology equipment for the development of a new variety can be made up of dozens of experts in biotechnology. Without this process, Ecuador would not be able to keep the dynamism and keep up with the international demand (National Plan for Good Living, 2013-2017)

\section{Pesticides}

Greenhouses.

Technification and irrigation technology. The technological Irrigation Project (PIT) allows farmers to use water more 
efficiently through irrigated irrigation infrastructure, in addition to receiving technical support to strengthen the organization of the association, develop environmental and productive economy practices, as well as support the marketing of their products. WB World Bank (2017).

\section{$\underline{\text { Harvesting }}$}

\section{Mainstream}

Expoflor is the main actor of the cluster in Ecuador because they are the principal association that gather growers and exporters of flowers in Ecuador. Their mission is to represent the Ecuadorian flower sector and meet the needs of the partners, through fast and effective services that help them generate added value in business, framed in social and environmental standards.

\section{Down Stream}

Logistics, packaging, and marketing are three very important activities in this cluster. They are related to the final cost of the flower, and key elements of the final price and the competitiveness of the industry.

\section{Five force analysis of the Ecuadorian flower Industry}

As it has been seen throughout the present, Ecuador is in the 3rd position in exporters of the world in flowers, sharing the first positions with Holland, Colombia, Kenya, and China, by what next is a graph of the analysis of the Five Porter Forces regarding their status. 


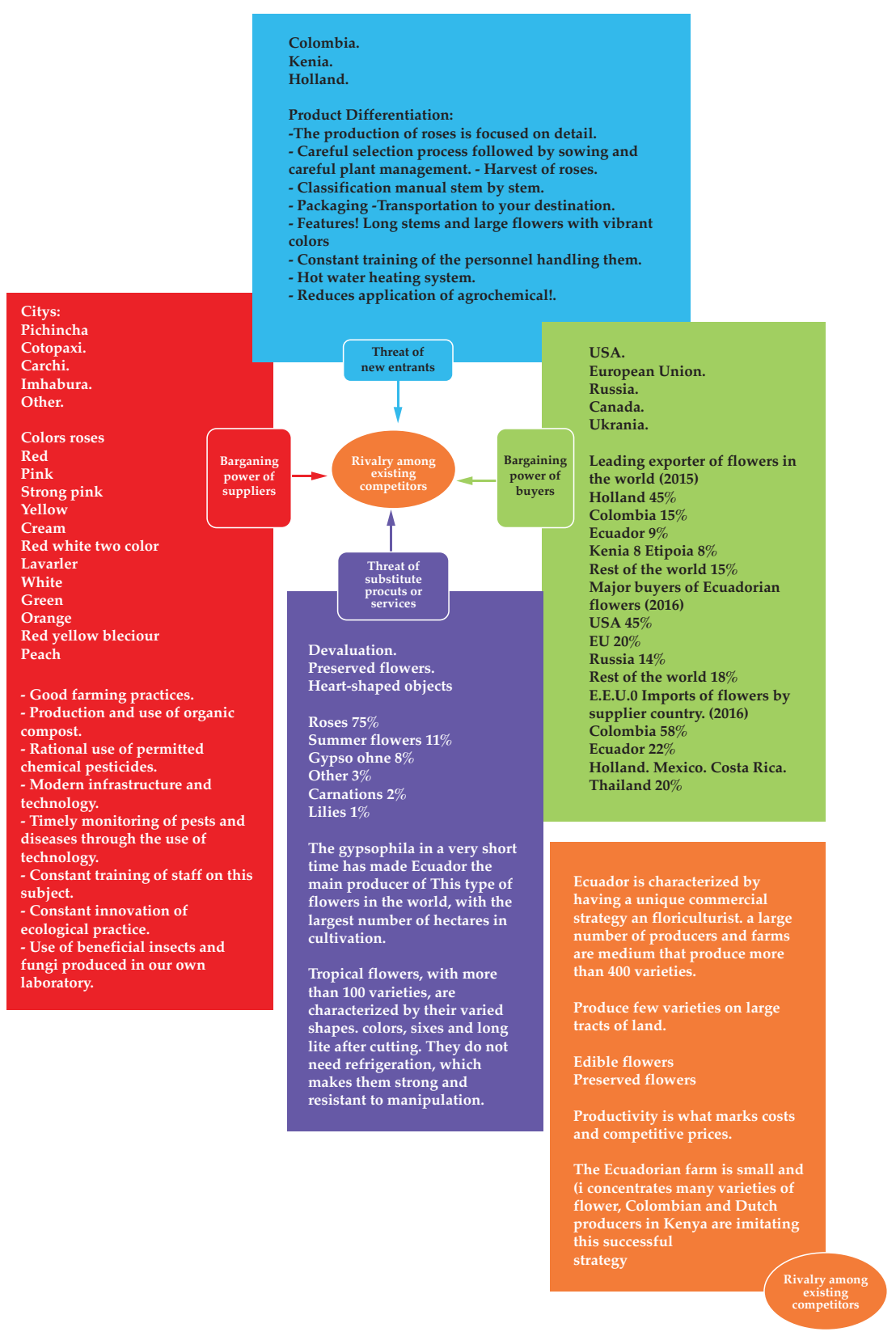

Figure 25. Five force analysis of the Ecuadorian flower Industry

Source: Prepared by the authors based on Porter (2008). 


\section{$\underline{\text { Rivalry among existing competitors }}$}

The export market of roses in Ecuador is one of the most important worldwide, has been characterized by size, color and its more than 400 varieties of roses, currently standing out in the production of edible flowers and preserved flowers. One aspect to emphasize is that Ecuadorian producers are small farms where many varieties of flowers are developed, which has been imitated by Colombia, the Netherlands and Kenya.

\section{$\underline{\text { Bargaining power of buyers }}$}

The Netherlands is in its first buyer of flowers, the United States is more focused on roses, followed by the European Union, but Colombia still ranks first in the importing United States.

\section{$\underline{\text { Bargaining power of suppliers }}$}

The characteristics that make Ecuador roses so beautiful have been achieved thanks to research on planting, reproduction, and the species itself, but the market has begun to demand better infrastructure and technology, the use of pesticides and other Chemical agents that have come to diminish the life of the planters, as well as the educational level of the people who oversee such treatments, a lot of talent has to leave Ecuador to other countries where they continue their research.

\section{Threat of new entrants}

The Ecuadorian producers of roses have focused on the detail, texture, color and length of roses stems, their harvesting strength has been specializing in cutting the flower at the right time to be packaged and have warehouses with temperature Ideal for 
maintaining them, in addition to the air delivery system that has also modified the design of the aircraft so that the flowers arrive fresh to their destination. The work is to continue working with people who intervene directly and indirectly on the flower to overcome Colombia in the first instance in the export of flowers.

\section{Threat of substitute products or services}

The market for roses is Ecuador's strongest export, followed by Summer flowers, Gyspophilias and other flowers. By the characteristics of the Gyspophilias they make a flower that occur in a lesser time than that of the roses and Ecuador is the first exporter of this flower to world-wide level, reason why the Ecuadorians should exploit more this segment, without neglecting the one of the roses.

\section{Synthesis of current situation}

\section{Process of the cultivation of the roses}

The roses belong to the Rosaceae family, whose scientific name is Rosa sp. The commercial varieties of roses that are known today are hybrids of species already disappeared. The flowers have long stems and attractive flowers arranged individually or with some lateral buds of medium or large size and numerous petals that form a visible central cone. No ornamental flower has been as appreciated as the rose, fact that places it first for its supply throughout the year (Pro Ecuador, 2016, p. 10). 


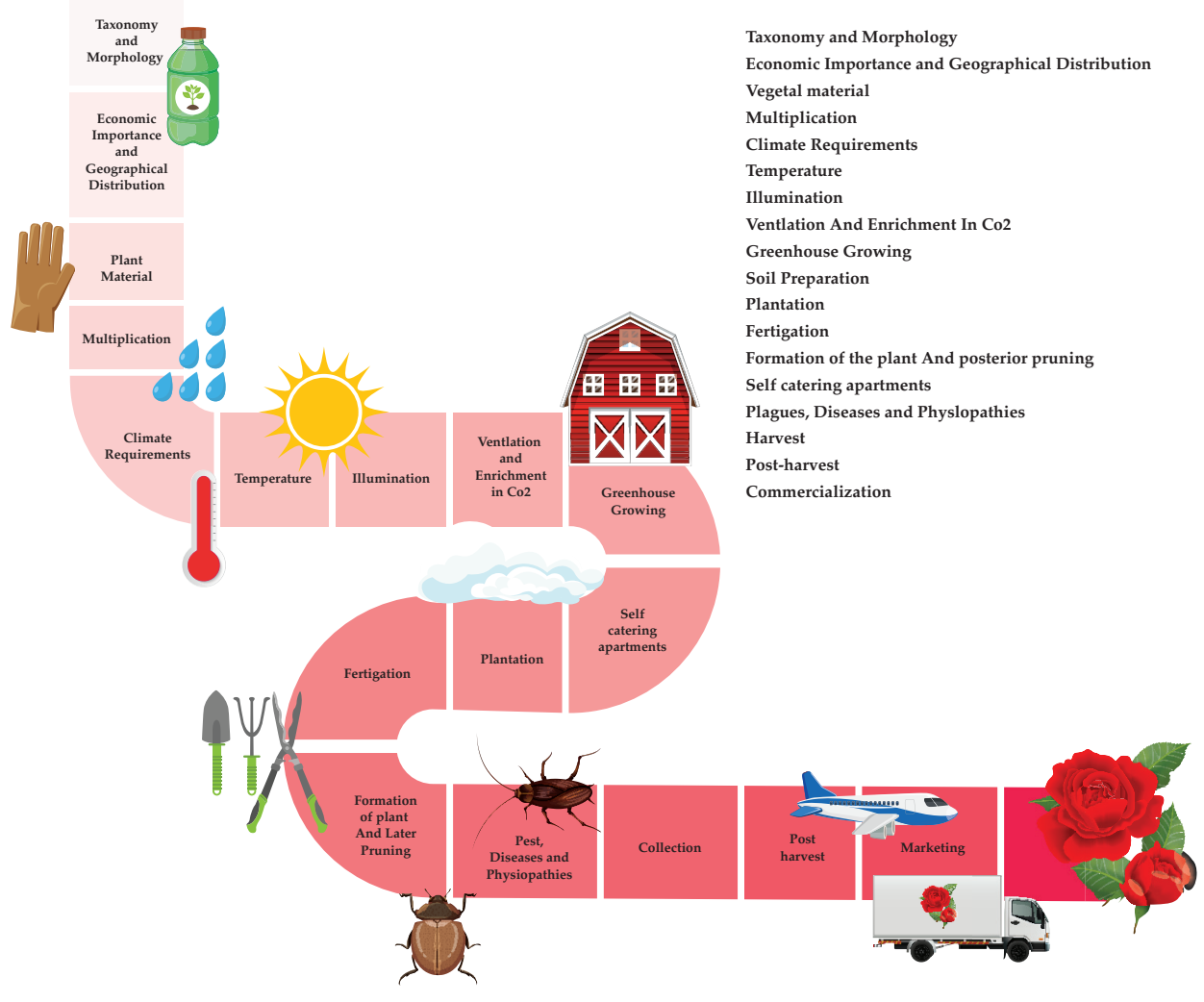

Figure 26. Process of the cultivation of the roses

Source: Prepared by the authors based on Porter (2008).

The desired qualities of roses for cutting, according to the tastes and demands of the market at each moment, are (Pro Ecuador, 2016, p. 12)

- Long and rigid stem: 50-70 cm, according to cultivation areas.

- Bright green foliage.

- Flowers: slow opening, good conservation in vase.

- Good flowering (equal to yield per foot or per m2).

- Good resistance to disease.

- Possibility of being cultivated at lower temperatures, in winter.

- Ability to grow without soil. 
The classification of the main crops is:

- Large products (80\% of production).

- Red (40-60\% of demand): First Red, Dallas, Royal Red, Grand Gala, Koba, Red Velvet, etc.

- Roses (20-40\% of the demand): Anna, Noblesse, Vivaldi, Sonia, Omega, Versilia, etc.

- Yellow (rising): Golden Times, Texas, Starlite, Live,

- Coktail 80.

- Oranges (rising): Pareo, etc.

- White: Virginia, Tineke, Ariana, etc.

- Bicolores: Candia, Simona, Prophyta, La Minuette, etc.

- Multiflores (spray): Mini (different colors), Golden Mini, Lidia (pink), Nikita (pink).

The propagation can be carried out by seeds, cuttings, rod grafts and bud grafts, although the latter is the most used method commercially. Seed reproduction is limited to new crops. The cuttings are selected from flower stems that have been allowed to fully develop the flower to ensure that the flower-producing bud is of the true type. Stakes with 1, 2 or 3 buds may be used, depending on the availability of plant material, although 3-buds are preferable because they have longer length and more nodal tissue at the base, thus reducing losses due to diseases (Pro Ecuador, 2016, p. 7)

A suitable humidity and a temperature in the medium of $18-21^{\circ}$ $C$ should be maintained. Under these conditions rooting occurs at 5-6 weeks, depending on the time of year and the nature of the stem. Subsequently transplant to pots of $7.5 \mathrm{~cm}$ or directly to the greenhouse (Pro Ecuador, 2016, p. 9). For most of the rose cultivars, the optimal growth temperatures are $17^{\circ} \mathrm{C}$ to $25^{\circ} \mathrm{C}$, with a minimum of $15^{\circ} \mathrm{C}$ overnight and a maximum of $28^{\circ} \mathrm{C}$ during the day (Pro Ecuador, 2016, p. 9) 
The growth index for most of the rose cultivars follows the total light curve throughout the year. Thus, in the summer months, when high luminous intensities prevail and long duration of the day, the production of flowers is higher than during the winter months (Pro Ecuador, 2016, p. 10)

With the cultivation of rose under greenhouse it is possible to produce flower in times and places in which otherwise would not be possible, obtaining the best prices. To do this, these greenhouses must meet minimum conditions: large dimensions $50 \times 20 \mathrm{~cm}$ and more, light transmission must be adequate, height must be considerable and ventilation in hot months should be good. In addition, heating during the winter is recommended, along with the installation of thermal blankets to conserve heat at night (Pro Ecuador, 2016, p. 12)

For the cultivation of roses, the soil must be well drained and aerated to avoid waterlogging, so that soils that do not meet these conditions must be improved in this sense, being able to use various organic materials. The planting season runs from November to March. In terms of planting distance, the current trend is to plant in 4 rows $(60 \times 15 \mathrm{~cm})$ or 2 rows $(40 \times 20$ or $60 \times 12.5$ $\mathrm{cm})$ with corridors at least $1 \mathrm{~m}$. This allows easier maintenance and lower investments (Pro Ecuador, 2016, p. 11)

The fertilization is done through irrigation, considering the subscriber fund provided, if it has been made. Subsequently it is also convenient to control the parameters of $\mathrm{pH}$ and electrical conductivity of the soil solution as well as conducting foliar analyzes (Pro Ecuador, 2016, p. 10)

The pests that attack the roses are: Red spider (Tetranychus urticae), Green aphid (Macrosiphum rosae), Nematodes 
(Meloidogyne, Pratylenchus, Xiphinema), Trips (Frankliniella occidentalis) and diseases that affect crops are Downy mildew or blight (Peronospora sparsa) Oidium (Sphaerotheca pannosa), Roya (Phragmidium disciflorum), Gray mold or botrytis (Botrytis cinerea), Galls or tumors (Agrobacterium tumefaciens) and the Foliar mosaic (Pro Ecuador, 2016, p. 7)

There are also the physiopathies that are the fall of the leaves that can be derived from different causes, from the sudden change in the growth, phytotoxicities frequently caused by herbicides of the type of phenoxides, that can produce severe symptoms of distortion and wrinkling of leaves and young stems. Sometimes petals appear shorter than normal and in excessive numbers, which in some places is known as "bull's head". The thrips are blamed for these symptoms, although it is common for these flowers to appear in the absence of thrips on very vigorous stems (Pro Ecuador, 2016, p. 12)

Generally, the cut of the flowers takes place in different periods, depending on the time of collection. In conditions of high luminosity during the summer, most of the varieties are cut when the sepals of the chalice are reflexes and the petals have not yet been deployed. However, the cut of the flowers during the winter is realized when they are more open, although with the two outside petals without unfolding. If they are cut too immature, the heads may wither, and the flower will not harden (Pro Ecuador, 2016, p. 9)

In the post-harvest several factors intervene, firstly it is necessary to take into account that each variety has a different cut point and therefore the level of maturity of the button and the peduncle will be decisive for the later evolution of the flower, once cut (Pro Ecuador, 2016, p. 9). Once the flowers are cut, the factors that 
can act on their wilt are difficulty of absorption and displacement of water by conducting vessels, inability of floral tissue to retain water and variation of intracellular osmotic concentration (Pro Ecuador, 2016, p. 10)

The cut stems are placed in trays or buckets with nutritive solution, removing them from the greenhouse as soon as possible to avoid wilting by transpiration of the leaves. They are immersed in a hot nutrient solution and cooled quickly. Before forming bouquets, place the flowers in water or in a nutrient solution (Pro Ecuador, 2016, p. 12) Once the flowers are removed from the store, the leaves and spines are removed from the bottom of the stem. Later the stems are classified according to lengths, discarding those curved or deformed and damaged flowers. (Pro Ecuador, 2016, p. 12)

The classification by length of stem can be done manually or mechanized. Currently there are numerous roses processors that perform the calibration. These machines have several selectors for the different lengths. Its use reduces labor (Pro Ecuador, 2016, p. 12). Finally, the branches are formed by tens that are sheathed in a plastic film and are returned to their store for an additional cooling $\left(4-5^{\circ} \mathrm{C}\right)$ before packaging, since the cut rose needs a few hours of cold before being Commercialized (Pro Ecuador, 2016, p. $12)$.

\section{Strategy: cluster value proposition}

According to Michael Porter, strategic positions emerge from three distinct sources, which are not mutually exclusive and often overlap. Two of them apply for our investigation and recommendations: 
Positioning can be based on producing a subset of an industry's products or services: Ecuador has 10 research labs focused on the breeding, which means the creation of new varieties, unique in the world, capable to adapt to different segments and characterized by its extraordinary beauty and quality. Most of these labs are based in Europe. We recommend investing in R\&D to keep the investigation process within the country, adding a competitive advantage.

Needs-based positioning: The roses cluster knows its groups of customers have different needs on different occasions, and its able to provide the necessary rose. Through R\&D, it would be possible not only to be aware of the needs, but to determine the market tendency. Considering that strategy is the creation of a unique and valuable position, involving a different set of activities, the one used by Ecuador should be to provide the most beautiful roses on earth, harvested and transported by a high-quality process involving shared value for all the participants.

Recommendations derived from the analysis including three relevant projects, including a shared value project.

\section{Project number one: Shared value project}

The concept of shared value focuses on the connections between societal and economic progress and has the power to unleash a wave of global growth. According to the investigation, the need to attend societal needs is evident. So, this is what we recommend:

- To redefine the productivity in the value chain and take in account the health and wellbeing of the work force, especially the women, who nowadays suffer the consequences of the use of pesticides. 
- To focus on the preparation of social entrepreneurs who are aware of the local needs.

- To link the education received in universities to the cluster needs, to take advantage of the generated knowledge and lead to a capable workforce.

- To invest more in Research and Development, attending needs related to energy use, environmental impact, water use, employee health, worker safety and employee skills.

- To establish a significant collaboration between universities and rose plantations.

- Investment in a wellness program of the families that are part of the value chain.

\section{Project number two}

During the research of this work and the information about the cluster, they were identified that one of the things that can help to improve this cluster is de invest on research and development. It is recommended research and development in the use of organic pesticides or in an alternative that protect the flowers but also the people and the environment. They need to be more responsible with the use of pesticides and bet for sustainability in their process.

It is also recommending investing in research and development in breeding, they do it now but in foreign countries and with foreign talent. We think that in Ecuador they have talent people that can focus on helping the cluster to be more competitive. If the Government, the universities, the actors of the cluster work together they can be more success and they can innovate together and be more competitive around the world like Holland. 


\section{Project number three}

And the last area in research and development that we think is especially important is in the creation of sub clusters that help to compete in international markets for example in the preserved roses that they are beginning to develop. One of those that could be developed more is in the market of the Gyspophilias, in which they are the first place, to exploit more this species, not to compete with the one of the roses, but to complement. The edible and perpetual roses have been growing more in the taste of the consumer, so you can take advantage of the market, as was the case of the Beauty and the Beast, to launch in these or generate new events to continue keeping the roses in the minds of consumers. 


\section{Conclusions}

Nowadays the Ecuadorian flower cluster, especially the roses subcluster rely on the quality of their products, though it is necessary to invest not only in visible infrastructure but in know how such as Holland and other countries, creating shared value. Though it is important to generate revenues the long-term goal should be the wellbeing of people in Ecuador.

Ecuador has experienced great achievements in the flower industry, the creation of new species, the abolition of child labor in this field and the research of edible and perpetual flowers, but the challenge has been difficult, since the currency Has suffered falls due to the change towards the dollar, an element that did not affect its two major competitors in the same way, and more specifically with respect to Colombia, who, by geographic situation, represents its main competitor in the export of roses To the United States, Europe and Asia.

There are still challenges, as in the case of research carried out in other countries and not directly in Ecuador, derived from corruption and lack of support for the education system, in addition to growing pressure from environmental protection agencies and Worker to reduce the impact of mortality and to the land itself. However, it has been maintained in the top 3 export locations, which now with the development in crops in Kenya and China, should be careful strategies and improving the processes so that these two do not overturn in sales. 


\section{References}

Association of Banks of Ecuador (ASOBANCA 2015 - 2016). Economic and Banking Environment. Annual Report

BBC News (2016). The impressive figures of the largest flower market in the world. Retrieved from: https://bbc.com/ future/

BI. Directorate of Intelligence and Investments. (2016). World floriculture map 2016: Equator countries gathering speed. Retrieved from: https: / / research.rabobank.com/far/en/ sectors

Central Bank of Ecuador. (BCE 2018). Gross domestic product 2004 - 2013. Retrieved from: https://contenido.bce.fin. ec/home1 / estadisticas / bolmensual / IEMensual.jsp

Economic Commission for Latin America and the Caribbean. (2016). Economic Survey of Latin America and the Caribbean. The 2030 agenda for sustainable development and the challenges of financing for development. Retrieved from: https: / / cepal.org/ sites / default/ files

El Comercio (2017, march 3rd). 8000 Ecuadorian roses at the premiere of Beauty and the Beast in Los Angeles. El Comercio.

Ecuadorian Institute of Intellectual Property. (IEPI 2014). Annual operative plan. Retrieved from: https:// derechosintelectuales.gob.ec/wp-content

Ecuadorian Institute of Social Security. (IESS 2017). Statistics 2004 - 2016. Statistics. Retrieved from: https://sart.iess.gob. ec/SRGP

National Institute of Statistics and Censuses. (INEC 2016). Educational level of Ecuadorians. Retrieved from: https: / / / estadisticas / ec

National Plan for Good Living, (2013-2017). National Development Plan. Retrieved from: https: / observatorioplanificacion. cepal.org / es / planes / plan-nacional-del-buen-vivir-20132017-de-ecuador 
Porter, M. (2008). On Competition. Competitive solutions to societal problems (pp. 347 - 405). USA: Harvard Business Press.

Porter, M., Ramírez, J. \& Van, F. (2013). At Harvard Business School. The Dutch Flower Cluster. 1-32.

Pro Ecuador. (2016). Statistics of Export Evolutions by Sector. Retrieved January 12, 2016, from Pro Ecuador: https: / / www.proecuador.gob.ec/exportadores/publicaciones / estadisticas-por-sector /

WB World Bank (2017). Technified irrigation, an option that improves the quality of life of small and medium farmers in Ecuador. Retrieved from: https://www.worldbank. org / en / news / feature / 2017 / 05 / 04 / riego-tecnificadoecuador

World Economic Forum. (2016). The Global Competitiveness Report. Annual Export Report, 3. Insight Report, 158. 


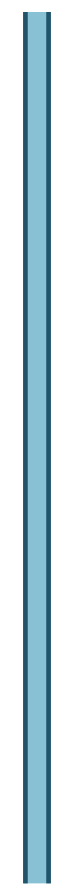

Annexes 
Appendix 1. Agricultural products cluster-Ecuador

\begin{tabular}{|c|c|c|c|c|c|c|c|c|c|c|c|c|c|c|c|c|c|c|c|}
\hline & \multirow{6}{*}{ 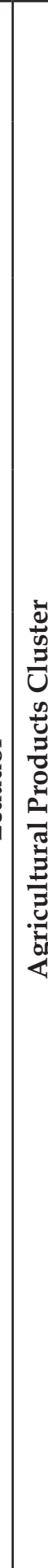 } & \multirow{6}{*}{ 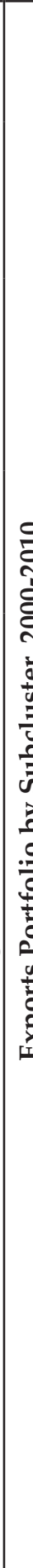 } & ت્ّ & 응 & 옴 & 옹 & 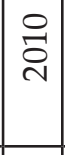 & 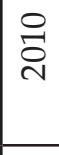 & $\begin{array}{l}\circ \\
\stackrel{2}{2}\end{array}$ & 옹 & 옹 & 옹 & 융 & 웅 & 옹 & 옹 & 옹 & 옹 & 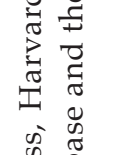 \\
\hline & & & 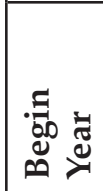 & \& & \&ి & §ి & 8 & 8 & §ి & \& & \& & \& & 8) & \& & ষ্ণ & \&્ત & 8 & \& & 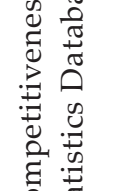 \\
\hline & & & 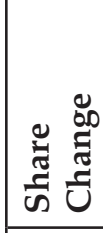 & $\begin{array}{l}50 \\
8 \\
0 \\
0\end{array}$ & $\begin{array}{l}\dot{0} \\
8 \\
8 \\
0\end{array}$ & $\begin{array}{l}\dot{0} \\
\dot{8} \\
0\end{array}$ & $\mid \begin{array}{l}80 \\
8 \\
8 \\
0\end{array}$ & $\begin{array}{l}\infty 0 \\
\infty \\
1 \\
0\end{array}$ & $\begin{array}{l}0 \\
\vdots \\
0 \\
0\end{array}$ & $\begin{array}{l}80 \\
\stackrel{0}{0} \\
0 \\
0\end{array}$ & 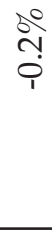 & $\begin{array}{l}D^{0} \\
10 \\
0 \\
0\end{array}$ & $\begin{array}{l}5_{0}^{0} \\
\text { రे } \\
0\end{array}$ & $\begin{array}{l}0 \\
0 \\
0 \\
0\end{array}$ & $\begin{array}{l}0 \\
0 \\
0 \\
0 \\
0\end{array}$ & $\begin{array}{l}\stackrel{D}{0} \\
\stackrel{y}{t}\end{array}$ & के & $\begin{array}{l}80 \\
8 \\
8 \\
0\end{array}$ & 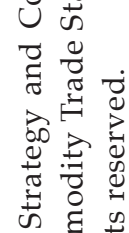 \\
\hline & & & సँّ & $\begin{array}{l}\dot{S}^{\circ} \\
8 \\
0\end{array}$ & $\begin{array}{l}\stackrel{0}{0} \\
8 \\
0\end{array}$ & $\begin{array}{l}\dot{8} \\
\stackrel{8}{8} \\
\stackrel{0}{0}\end{array}$ & $\begin{array}{l}\tilde{0} \\
0\end{array}$ & $\mid \begin{array}{l}20 \\
\frac{1}{2} \\
0\end{array}$ & $\begin{array}{l}\frac{D}{0} \\
\vdots \\
0 \\
0\end{array}$ & \begin{tabular}{l}
0 \\
\multirow{2}{*}{+} \\
0 \\
0
\end{tabular} & 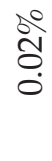 & $\begin{array}{l}D_{0}^{0} \\
10 \\
0 \\
0\end{array}$ & $\begin{array}{l}50 \\
10 \\
0 \\
0\end{array}$ & $\begin{array}{l}\stackrel{8}{\circ} \\
\text { 웅 }\end{array}$ & $\stackrel{0}{\circ}$ & $\begin{array}{l}\dot{\circ} \\
\text { ळ̆ }\end{array}$ & $\begin{array}{l}80 \\
\text { bे } \\
+1 \\
\text { cे }\end{array}$ & $\begin{array}{l}\text { sू } \\
\text { के } \\
\text { ?ं }\end{array}$ & 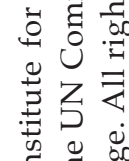 \\
\hline & & & 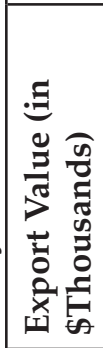 & $\begin{array}{l}8 \\
\stackrel{8}{\circ} \\
\dot{\leftrightarrow}\end{array}$ & 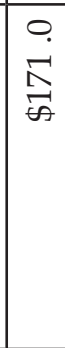 & $\begin{array}{l}8 \\
8 \\
8 \\
\infty \\
\infty\end{array}$ & 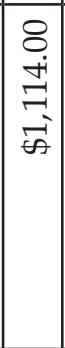 & 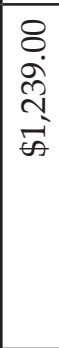 & 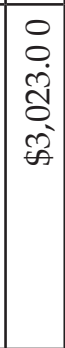 & 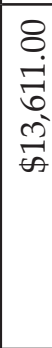 & 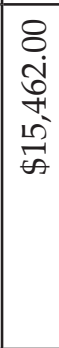 & 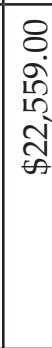 & $\begin{array}{l}8 \\
0 \\
0 \\
\hat{0} \\
\hat{2} \\
\text { సे } \\
0\end{array}$ & 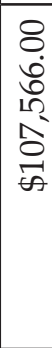 & 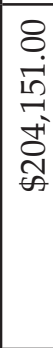 & $\begin{array}{l}8 \\
\text { के } \\
\text { के } \\
\text { à } \\
10 \\
0\end{array}$ & $\begin{array}{l}8 \\
8 \\
\infty \\
10 \\
1 \\
-1 \\
6 \\
6\end{array}$ & 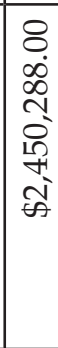 & 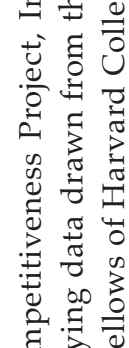 \\
\hline & & & 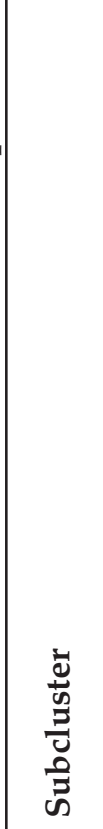 & $\frac{y}{u}$ & $\stackrel{0}{3}$ & 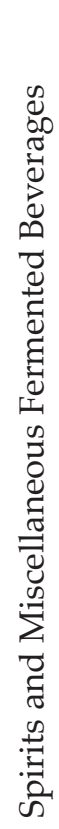 & 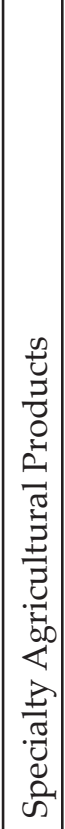 & 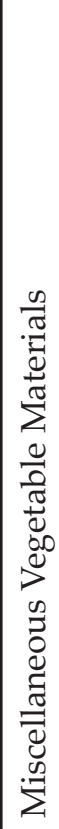 & 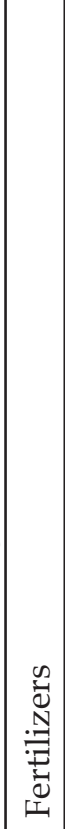 & 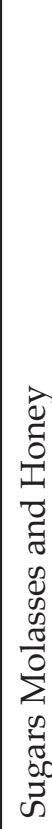 & 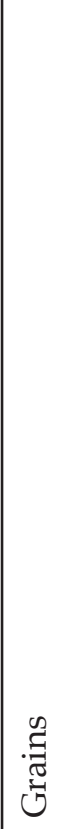 & 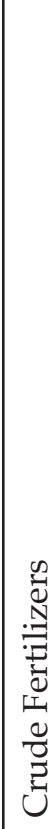 & 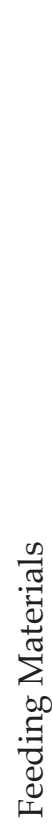 & 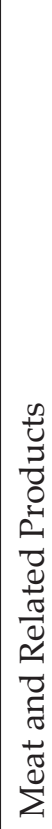 & 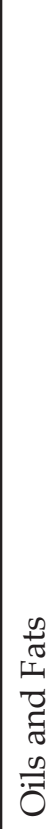 & 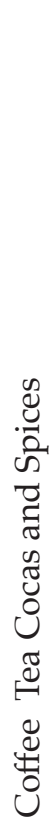 & 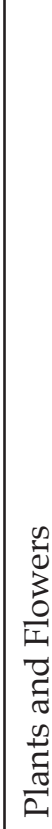 & 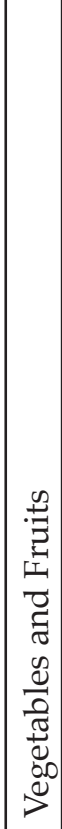 & 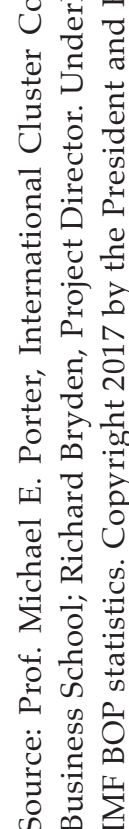 \\
\hline
\end{tabular}


Appendix 2. Agricultural products cluster - World exports by subclusters 2010

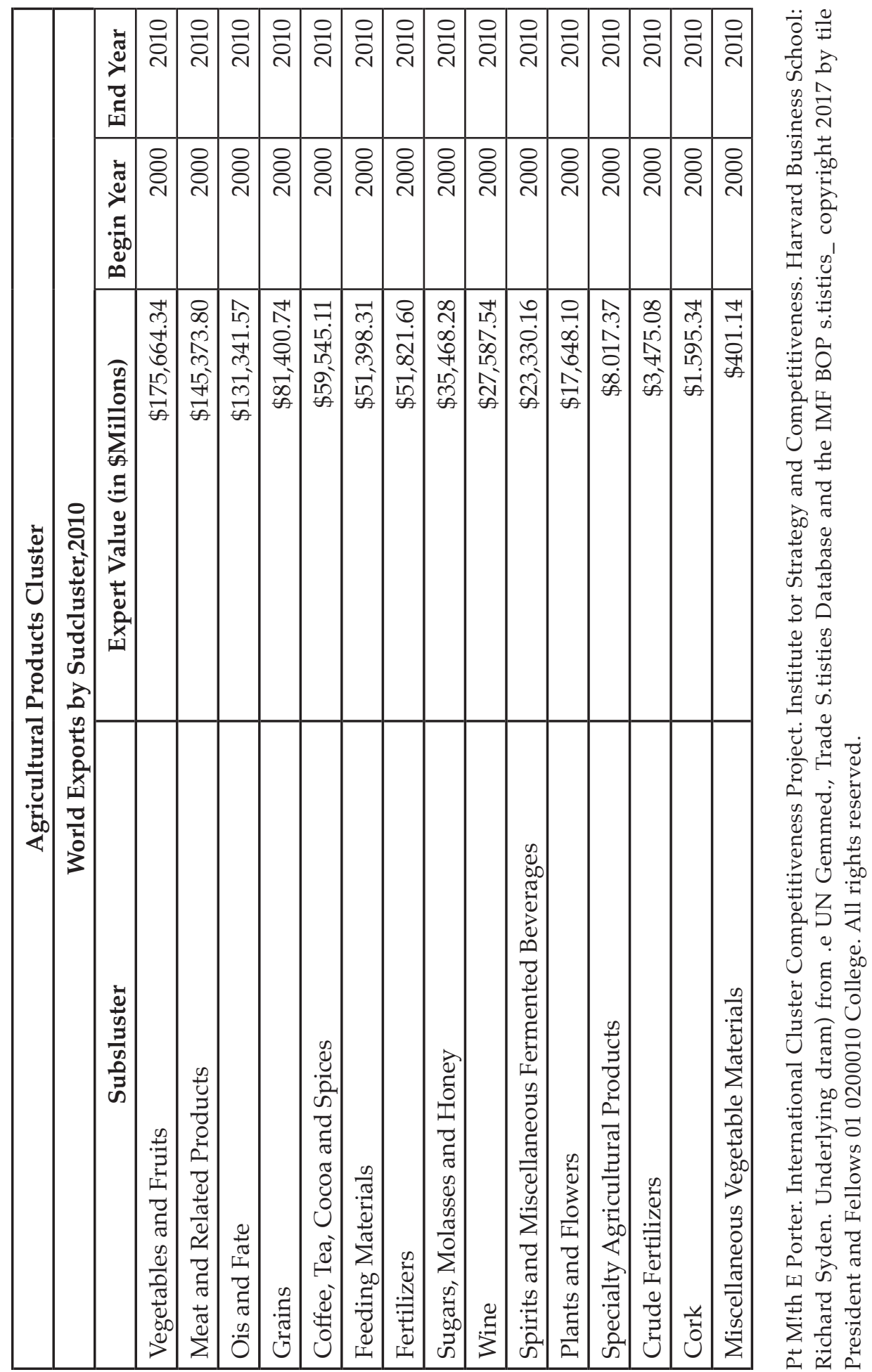


Appendix 3. List of exporters of cut flowers buds

\begin{tabular}{|c|c|c|c|c|c|c|c|c|c|c|c|c|}
\hline & 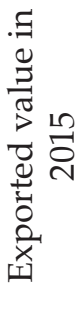 & $\begin{array}{l}\text { ڤn } \\
\text { Jै } \\
10 \\
\infty \\
\infty\end{array}$ & $\begin{array}{l}\text { నે } \\
\text { กิ } \\
\text { ๙ิ }\end{array}$ & $\begin{array}{l}\text { ळे } \\
\text { హे } \\
\infty\end{array}$ & 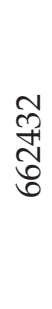 & $\begin{array}{l}8 \\
1 \\
10 \\
10\end{array}$ & $\begin{array}{l}\stackrel{\text { f }}{\rightarrow} \\
\text { }\end{array}$ & $\begin{array}{l}\stackrel{0}{0} \\
\stackrel{1}{1}\end{array}$ & $\begin{array}{l}\hat{\sigma} \\
\underset{+}{\not}\end{array}$ & $\begin{array}{l}\text { F } \\
\infty \\
\infty \\
\infty\end{array}$ & $\begin{array}{l}0 \\
\vec{\lambda} \\
\infty \\
\infty\end{array}$ & \\
\hline 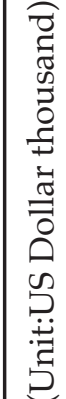 & 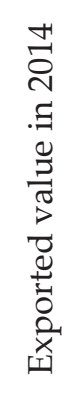 & 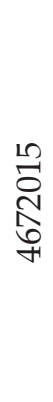 & 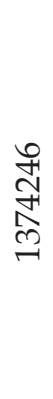 & $\begin{array}{l}\stackrel{\infty}{\mathbb{N}} \\
\infty \\
\sigma\end{array}$ & 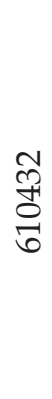 & $\begin{array}{l}3 \\
1 \\
10 \\
10 \\
10\end{array}$ & $\begin{array}{l}\text { ஓे } \\
\text { ळे } \\
\text { }\end{array}$ & $\underset{\infty}{N}$ & $\begin{array}{l}\text { న } \\
\text { ని } \\
\infty\end{array}$ & $\begin{array}{l}\text { ని } \\
\text { ర్ } \\
10 \\
\text { ND }\end{array}$ & $\frac{\infty}{\sigma}$ & 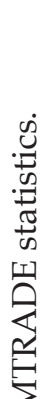 \\
\hline 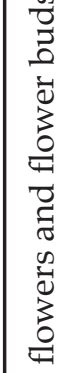 & 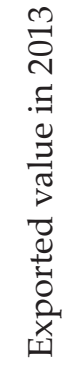 & 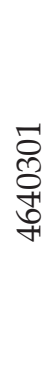 & 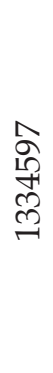 & $\begin{array}{l}\text { న } \\
\infty \\
\hat{\infty} \\
\infty\end{array}$ & 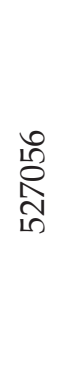 & 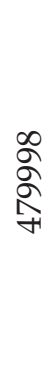 & 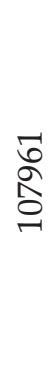 & $\begin{array}{l}\text { 帛 } \\
\text { مू }\end{array}$ & 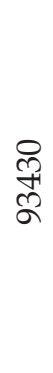 & $\begin{array}{l}\vec{\infty} \\
\stackrel{\infty}{0} \\
\infty \\
\sim\end{array}$ & 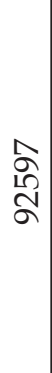 & 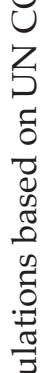 \\
\hline 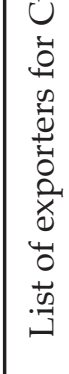 & 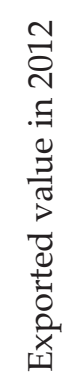 & 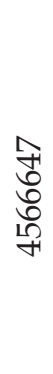 & $\begin{array}{l}\text { 용 } \\
8 \\
\text { 의 }\end{array}$ & 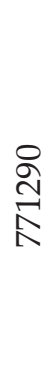 & \begin{tabular}{l}
\multirow{H}{*}{} \\
ద్ర \\
-
\end{tabular} & $\begin{array}{l}8 \\
0 \\
0 \\
\stackrel{1}{f}\end{array}$ & 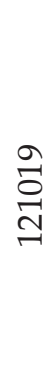 & $\begin{array}{l}\text { ஓ } \\
\curvearrowright \\
\infty\end{array}$ & $\begin{array}{l}\stackrel{\circ}{1} \\
\stackrel{+}{\infty}\end{array}$ & $\begin{array}{l}\frac{+}{n} \\
\stackrel{m}{\sim} \\
\stackrel{n}{N}\end{array}$ & $\begin{array}{l}\text { જे } \\
\infty \\
\infty \\
\infty\end{array}$ & 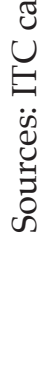 \\
\hline & 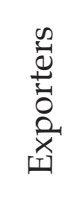 & 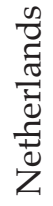 & $\begin{array}{l}\frac{\pi}{0} \\
\frac{0}{\tilde{0}} \\
\frac{0}{0} \\
ن\end{array}$ & $\frac{\tilde{0}}{\stackrel{\tilde{\sigma}}{\tilde{J}}}$ & 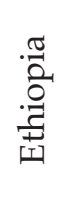 & $\underset{\widetilde{\Xi}}{\widetilde{\Xi}}$ & $\frac{\sqrt[\pi]{\omega}}{\stackrel{\vec{\pi}}{\frac{\pi}{d}}}$ & . & $\frac{\lambda}{ \pm}$ & $\frac{\Xi}{\Xi}$ & 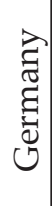 & \\
\hline
\end{tabular}


Appendix 4. List of importers of cut flowers and flowers buds

\begin{tabular}{|c|c|c|c|c|c|c|c|c|c|c|c|c|}
\hline & 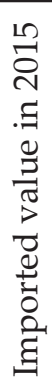 & $\begin{array}{l}\infty \\
\stackrel{\infty}{v} \\
\stackrel{\infty}{+} \\
\stackrel{\sim}{\sim}\end{array}$ & 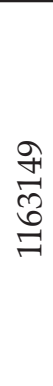 & $\begin{array}{l}\text { N̂ } \\
\text { م⿱ } \\
0 \\
0 \\
-\end{array}$ & 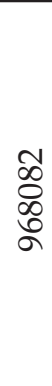 & $\begin{array}{l}\infty \\
\text { ô } \\
\text { مे }\end{array}$ & $\begin{array}{l}\text { on } \\
\text { 员 } \\
\text { m }\end{array}$ & $\begin{array}{l}\text { on } \\
\text { d̦ } \\
\text { ñ }\end{array}$ & $\begin{array}{l}3 \\
0 \\
\infty \\
\infty \\
\end{array}$ & $\begin{array}{l}\text { బ } \\
\tilde{N} \\
\stackrel{1}{0}\end{array}$ & 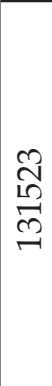 & \\
\hline 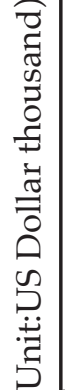 & 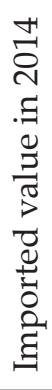 & $\begin{array}{l}\text { ڤે } \\
\text { సે } \\
\text { స్ }\end{array}$ & 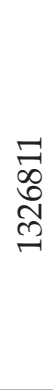 & $\begin{array}{l}\stackrel{+}{N} \\
\stackrel{\sigma}{\Xi} \\
=\end{array}$ & $\begin{array}{l}\text { ్ㅡ } \\
\text { ूू }\end{array}$ & 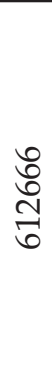 & $\begin{array}{l}\infty \\
+ \\
\infty \\
\stackrel{+}{+} \\
\stackrel{+}{+}\end{array}$ & 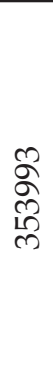 & $\begin{array}{l}\hat{\sigma} \\
\text { oర } \\
\text { ర్ }\end{array}$ & $\begin{array}{l}\text { ̊ి } \\
\text { مू } \\
\text { مิ }\end{array}$ & 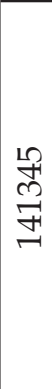 & 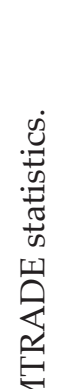 \\
\hline 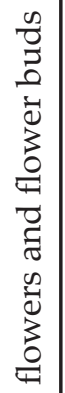 & 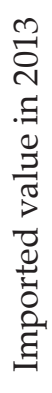 & 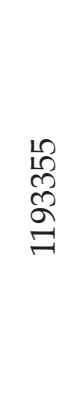 & $\begin{array}{l}\infty \\
\stackrel{N}{N} \\
\stackrel{\sim}{\sim} \\
\stackrel{\sim}{N}\end{array}$ & 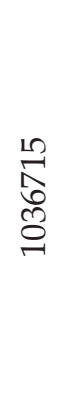 & 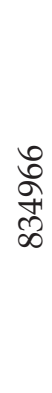 & 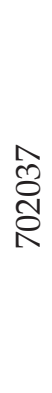 & 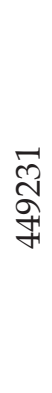 & $\begin{array}{l}\bar{\delta} \\
\delta \\
\infty \\
\infty \\
\text { n) }\end{array}$ & $\begin{array}{l}\text { स } \\
\text { o } \\
\curvearrowright \\
\sigma\end{array}$ & 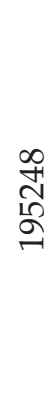 & $\begin{array}{l}\stackrel{m}{F} \\
\stackrel{9}{+}\end{array}$ & 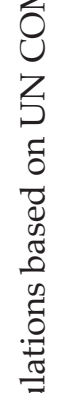 \\
\hline 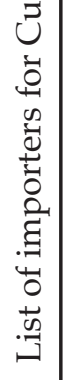 & 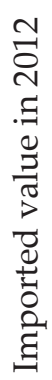 & 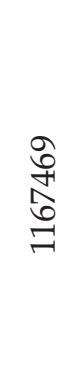 & 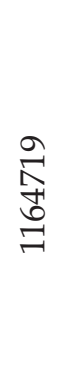 & $\begin{array}{l}\text { क } \\
\text { O } \\
\text { 岕 } \\
\sigma\end{array}$ & $\begin{array}{l}\text { f } \\
\text { D } \\
\nwarrow \\
\end{array}$ & $\begin{array}{l}\text { oి } \\
\hat{6} \\
+ \\
1\end{array}$ & 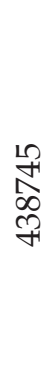 & \begin{tabular}{l} 
ND \\
$\stackrel{2}{F}$ \\
\multirow{F}{*}{}
\end{tabular} & 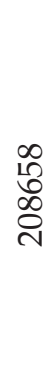 & $\begin{array}{l}\text { 어 } \\
\infty \\
\infty \\
\infty \\
-1\end{array}$ & 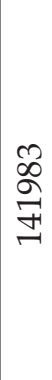 & 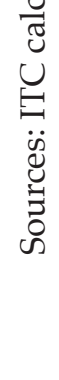 \\
\hline & 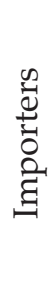 & 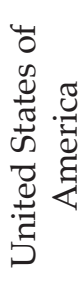 & 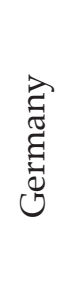 & 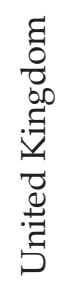 & 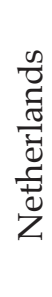 & 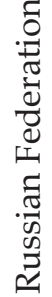 & 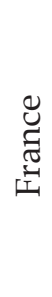 & $\begin{array}{l}\tilde{\Xi} \\
\text { ప్ } \\
\text { I }\end{array}$ & $\frac{\lambda}{ \pm}$ & 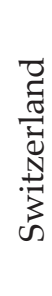 & $\frac{\tilde{J}}{\tilde{J}}$ & \\
\hline
\end{tabular}


Appendix 5. Main data of Ecuador

\begin{tabular}{|c|c|c|c|c|c|c|c|}
\hline 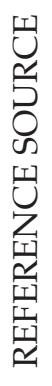 & 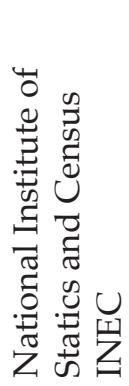 & 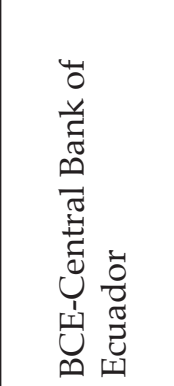 & 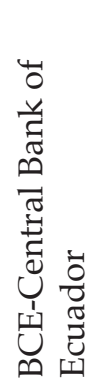 & 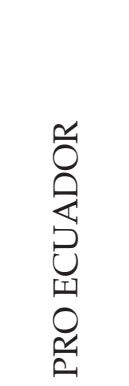 & 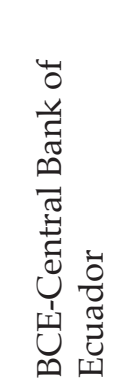 & 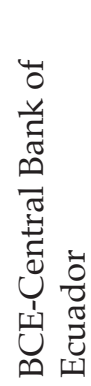 & 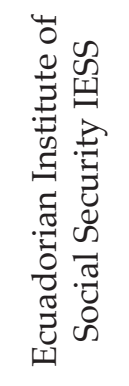 \\
\hline $\begin{array}{l}0 \\
\stackrel{\sim}{\sim}\end{array}$ & 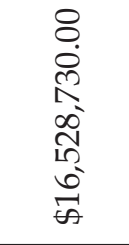 & 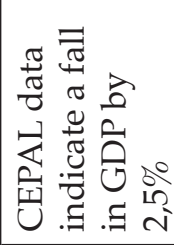 & $\begin{array}{l}8 \\
10 \\
0 \\
8 \\
8 \\
8 \\
0\end{array}$ & 0 & $\begin{array}{l}\infty \\
\stackrel{\infty}{F} \\
\dot{F}\end{array}$ & $\begin{array}{l}\cong \\
\text { ڤొ } \\
\text { के }\end{array}$ & $\begin{array}{l}8 \\
8 \\
8 \\
8 \\
\text { i } \\
\text { ô }\end{array}$ \\
\hline$\stackrel{20}{\stackrel{2}{\sim}}$ & 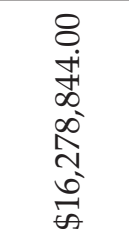 & 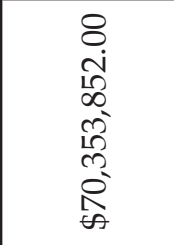 & $\begin{array}{l}8 \\
0 \\
\sigma \\
\sigma \\
\infty \\
\infty \\
\infty\end{array}$ & 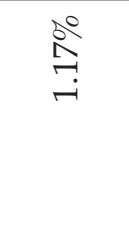 & $\begin{array}{l}\text { ๙ } \\
\text { ஸ̂ }\end{array}$ & 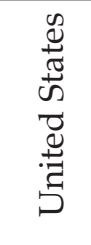 & $\begin{array}{l}8 \\
8 \\
8 \\
8 \\
0^{\circ} \\
0\end{array}$ \\
\hline$\underset{\sim}{\stackrel{+}{\sigma}}$ & 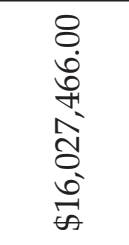 & 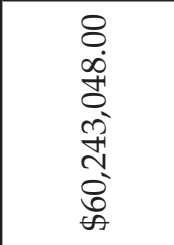 & \begin{tabular}{l}
8 \\
i \\
\multirow{1}{}{} \\
$\infty$ \\
$\sigma$ \\
$\sigma$ \\
$\infty$
\end{tabular} & 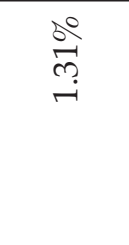 & $\begin{array}{l}\not \\
\infty \\
\infty \\
\infty\end{array}$ & 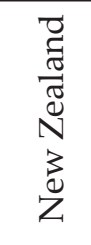 & $\begin{array}{l}8 \\
8 \\
8 \\
8 \\
\text { ஸे }\end{array}$ \\
\hline 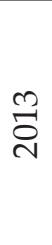 & 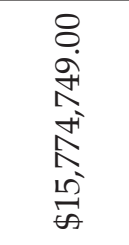 & 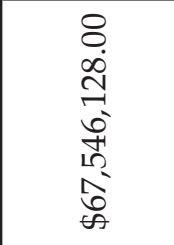 & $\begin{array}{l}8 \\
8 \\
\dot{1} \\
\stackrel{2}{1} \\
\infty \\
\infty \\
\infty \\
0\end{array}$ & $\stackrel{\text { @̊ }}{\stackrel{\text { ஸे }}{r}}$ & 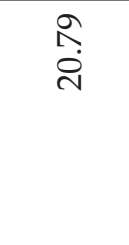 & $\begin{array}{l}\text {.ే } \\
\text { ڤิ }\end{array}$ & $\begin{array}{l}8 \\
\dot{8} \\
8 \\
\stackrel{8}{0} \\
\text { - }\end{array}$ \\
\hline 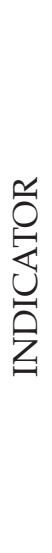 & 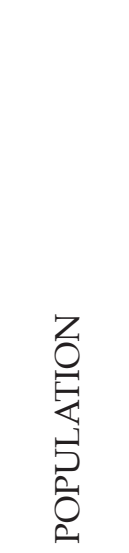 & जि & 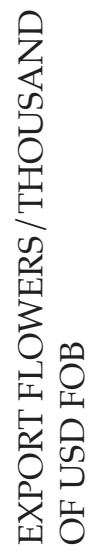 & 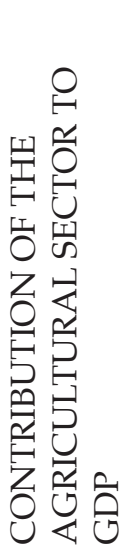 & 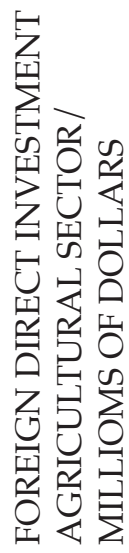 & 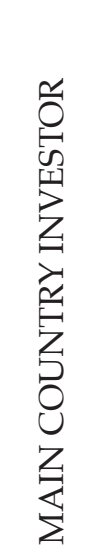 & 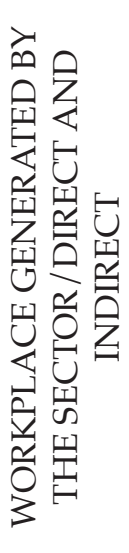 \\
\hline
\end{tabular}




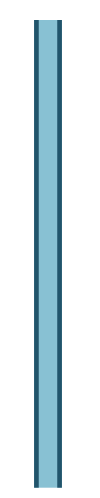

\section{CAPÍTULO 4}

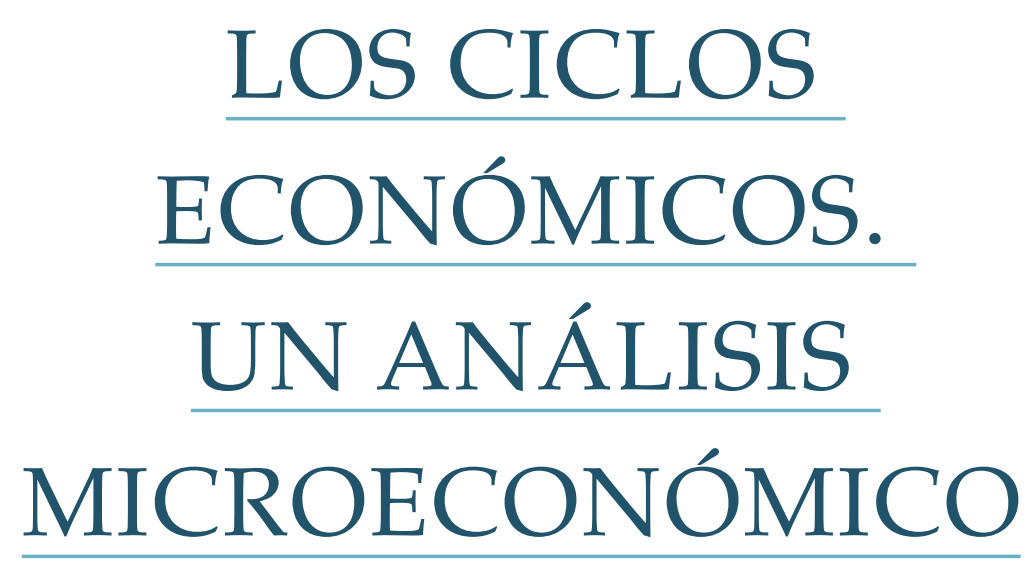

Eduardo G. Zurita-Moreano

María G. González-Bautista 


\section{Resumen}

Se pretende dar una fundamentación teóríica de los ciclos económicos que son fenómenos específicos de las economías basadas en el dinero y la actividad comercial. La existencia de diferentes ciclos en el conjunto de actividades que integran el sistema económico determina la naturaleza misma del capitalismo. En este sentido, las fluctuaciones son uno de los aspectos claves del comportamiento no estacionario de las economías de mercado donde los agregados económicos como el producto, el empleo y las inversiones tienen variaciones aperiódicas de diferentes duraciones e intensidad. En esencia se trata de reconocer que, si bien existe un ciclo general, en el cual se condensa el movimiento de la actividad económica agregada, también ocurren ciclos específicos, de acuerdo con las características propias de cada actividad. En los resultados de la presente investigación se destacan las teorías de los ciclos económicos haciendo énfasis en la teoría del ciclo económico real.

Palabras clave: actividad económica, ciclo económico, agregados económicos, teorías microeconómicas. 


\section{Introducción}

Snowdon y Howard (2005) plantean que uno de los principales objetivos de la macroeconomía moderna es determinar y comprender las causas de los agregados económicos, como el producto interno bruto, el consumo, la inversión, el desempleo, la inflación y el intercambio internacional. Específicamente, la macroeconomía estudia los determinantes e impactos de las fluctuaciones de la actividad económica en el corto plazo (ciclos económicos) y los determinantes de la senda de largo plazo de la actividad económica (crecimiento económico).

Desde hace mucho tiempo las escuelas de pensamiento económico han tratado de explicar este comportamiento de los agregados económicos con el fin de articular políticas de crecimiento. Así, la escuela keynesiana trató de explicar el alto desempleo de la crisis de 1930 debido a una demanda agregada insuficiente, los neoclásicos indicaban la existencia de una relación inversa entre el desempleo y la inflación, o los monetaristas ponen énfasis en el mercado de dinero como variable principal en los ciclos económicos. En las últimas décadas se han desarrollado nuevas escuelas como: la nueva economía clásica, la escuela de los ciclos económicos reales, la nueva economía keynesiana y la reciente nueva síntesis neoclásica. Todas han elaborado marcos teóricos para brindar explicaciones sobre el comportamiento de las variables económicas (Galindo y Montecinos, 2017).

Esta investigación busca analizar, desde diferentes perspectivas del pensamiento económico, los ciclos económicos. Así, la escuela clásica que sostiene que los cambios en los precios relativos, especialmente del salario real, son el mecanismo de optimización de la economía, y que el desempleo no refleja la falta de ajuste salarial, sino que este puede darse porque la legislación impide 
que el mercado se equilibre. Para los keynesianos el mercado laboral tarda en equilibrarse, ya que el salario nominal no se ajusta rápidamente a los cambios de la dinámica económica, y sostienen además la inestabilidad de la demanda agregada.

Dentro de los economistas clásicos se citan a los monetaristas, cuyo máximo representante es Milton Friedman. Estos sostienen que los shocks económicos se relacionan con la inestabilidad de las políticas monetarias. Luego están los investigadores de las expectativas racionales con Lucas y Barro, quienes afirman que las políticas monetarias que afectan a la demanda agregada son una de las causas importantes del ciclo económico. Siguiendo por los fundamentos clásicos se mencionan a los teóricos de los ciclos económicos reales, los que indican que los shocks son de origen tecnológicos e impactan a la economía por el lado de la oferta.

Los neokeynesianos representados por Blanchard y Mankiw, han mantenido muchos de los supuestos de Keynes, pero han extendido su perspectiva indicando que los ciclos económicos podrían darse no solo por la demanda sino también por la oferta agregada. La nueva síntesis neoclásica con representantes como: Goodfried, King, Clarida, Gali y Gertler, quienes, desde finales de los noventa, desarrollan un conjunto de modelos que indican el vínculo entre los precios y la tasa de interés, bajo reglas de política monetaria, pero sin hacer énfasis en la cantidad total de dinero (Tobón, 2008).

El ciclo económico, según Burns y Mitchell (1946), se basa en la función de lucro, en donde la crisis es seguida por la depresión, la cual a su turno precede la recuperación, y esta última se transforma en prosperidad, la cual procrea una nueva crisis. Así, la descripción del ciclo puede iniciarse por cualquier fase. Una vez iniciada, cada fase tiende a desarrollarse en forma acumulativa. En ausencia de 
choques externos que podrían acelerar o retardar el movimiento acumulativo, los cambios ocurrirán, según Mitchell, "lenta pero seguramente" (Avella y Fergusson, 2003). Se destaca que la teoría de los ciclos económicos vuelve a ocupar un punto central cuando existen las crisis o recesiones. Sin embargo, el redescubrimiento de los ciclos encuentra a la ciencia económica cada vez mejor equipada con nuevas teorías, modelos, econometría, paquetes de investigación estadística y series de tiempo más detalladas para cada generación de investigadores.

El análisis de los ciclos económicos tiene una gran importancia debido a que a través de ello se observa la fase por la que esté atravesando una economía. Mediante este análisis, se puede determinar causas y efectos de la fase en la cual se encuentre el ciclo económico. En sí, el ciclo económico muestra las diferentes etapas económicas (producción, con sus variantes de desempleo, inflación y balanza comercial) por la que atraviesa un país con relación al tiempo. Su importancia radica en que se convierten en experiencia y al estudiarlos es posible prevenir o reafirmar situaciones a fin de suavizar dichos ciclos.

\section{Ciclos económicos}

Los ciclos económicos son un conjunto de fases por las cuales una economía atraviesa fluctuaciones de crecimiento o desaceleración en un período de tiempo. Cabe resaltar que, el inicio de una fase marca el fin de otra (Sevilla, 2015). La fluctuación económica se refiere a las variaciones intrínsecas de las variables macroeconómicas, ocasionadas por los distintos shocks que pueden afectar a la estructura de la oferta o demanda agregada. Estos shocks pueden ser exógenos o endógenos. Los primeros se relacionan con perturbaciones externas en variables económicas importantes como el precio de materias primas que tiene gran 
impacto en toda la economía, la tasa de interés internacional, los términos de intercambio que no dependen del manejo económico de los países. Por el contrario, los shocks endógenos se refieren a perturbaciones internas de las variables económicas como el déficit fiscal, tipo de cambio, la tasa de interés, políticas del mercado laboral, oferta monetaria entre otras, que pueden ser controladas a través de la política económica de los países (Llorca y Masó, 2016).

En ese sentido, Resico (2011) señala:

Los principales fenómenos a los que está sometida la economía de mercado es el de la existencia de ciclos económicos. Los ciclos o fluctuaciones cíclicas de la actividad económica pueden definirse como las oscilaciones de la actividad económica que dan lugar a la expansión y la contracción alternada de la economía en forma de eventos sucesivos. Esto implica que el crecimiento económico no es un proceso lineal de incremento en la cantidad de bienes y servicios producidos, sino que se presentan alternativamente épocas de auges y recesiones (pág. 179).

Así mismo, Resico (2011) explica que el ciclo económico se refiere a las fluctuaciones periódicas de la actividad económica en el contexto de la tendencia al crecimiento de largo plazo. El ciclo es la sucesión de períodos de expansión del crecimiento del producto, seguido de períodos de contracción. El ciclo implica cambios entre períodos de rápido crecimiento de la producción (recuperación y prosperidad) y períodos de relativo estancamiento o declive (contracción y recesión), producidos en el transcurso del tiempo. Estas fluctuaciones son medidas generalmente en términos del producto interno bruto de una determinada economía. 
Por su parte, Ramírez (2010) explica que un ciclo económico está compuesto por las fluctuaciones de las variables macroeconómicas más importantes de un país, mismas que a través de sus variaciones positivas o negativas permiten saber si la economía va en aumento o en declive o cuáles son los factores internos o externos que influyen para que la economía varíe de forma drástica o lenta, pero significante a través del tiempo.

Schumpeter (1976) en su libro Capitalism, Socialism, and Democracy, indica que la razón del capitalismo es el cambio económico y que por consecuencia jamás fue ni será alguna vez estacionario. Esto se demuestra en las variaciones que se dan en variables claves de la economía como el empleo, producción e inversión, indicando el comportamiento no estacionario del sistema capitalista.

Uno de los aspectos claves de la teoría económica es si los ciclos comerciales son inherentes y, por lo tanto, característica inamovible de las economías capitalistas o son el resultado de shocks externos actuando sobre sistemas económicos. Igor Matutinovic en su artículo Los fundamentos microeconómicos de los ciclos comerciales: De las instituciones a las redes autocataliticas, indica que hay dos posturas importantes sobre los orígenes de los ciclos económicos, los que creen que se debe a factores exógenos basados en golpes y endógenos fundamentados en la teorización evolutiva. Lo que lleva a concluir que si en un país no se cuenta con datos históricos acerca de sus ciclos económicos sería muy difícil mejorar su condición económica, así como estar prevenido para un acontecimiento negativo interno o externo que en el ámbito económico siempre se está expuesto a que esto ocurra. 


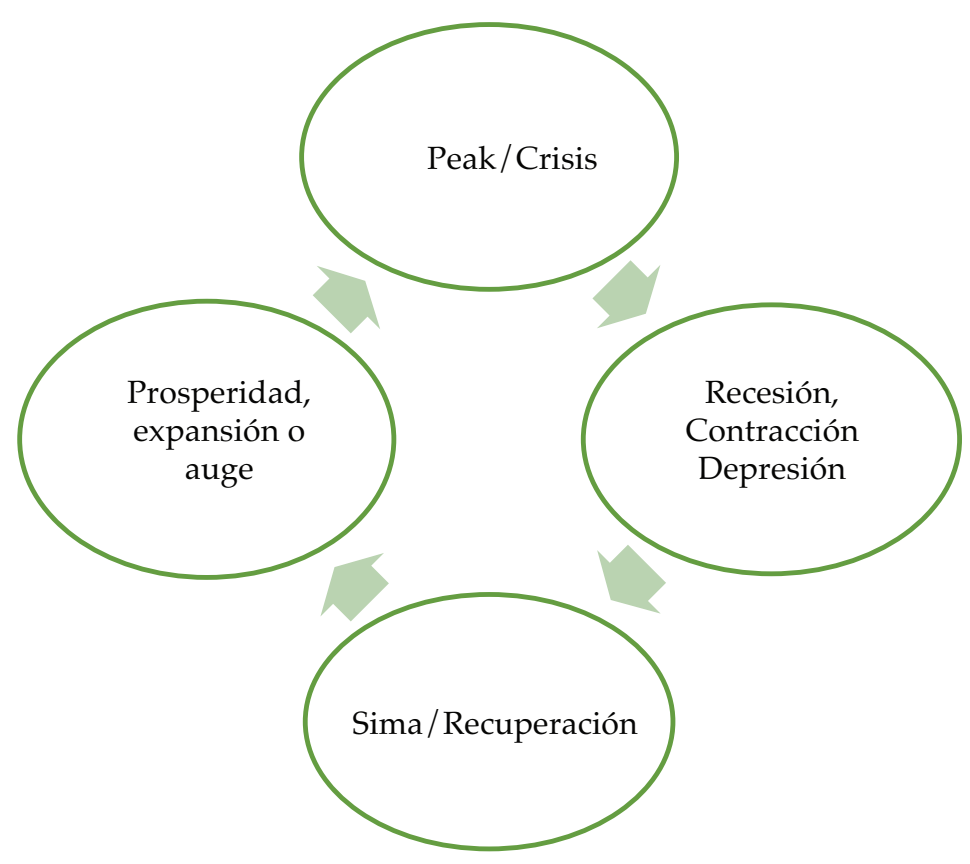

Figura 27. Fases de los ciclos económicos

Fuente: elaboración propia con base en Gaviola (2011).

Según Gaviola (2011) en los ciclos económicos se puede identificar de cuatro fases:

Recuperación: es el comienzo del ciclo, en el sentido que marca el fin de la caída previa en los indicadores de producto. Marca el comienzo de una nueva fase expansiva. Resurgen las inversiones que van a posibilitar el ascenso ulterior. Suelen ser períodos donde las contradicciones que llevaron a la crisis y recesión previas han sido resueltas o se ha encontrado una salida que permite recuperar la rentabilidad de las inversiones, y en consecuencia generar expectativas de beneficios futuros para los empresarios que emprendan negocios (Schumpeter, 1939). Las perspectivas son optimistas. En esta fase ocurre el comienzo de la recuperación del nivel de actividad. Generalmente existe un alto desempleo y bajos salarios. También suele existir importante capacidad ociosa 
de infraestructura y capital. Todos estos factores combinados permiten que, ante la recuperación de los precios, la producción vuelva a ser rentable (Schumpeter, 1939).

Prosperidad, expansión o auge:es la fase del ciclo en la cual crecen los indicadores de producto, crece la ocupación de la fuerza laboral y la utilización de los medios de producción (disminuye la capacidad ociosa). Crecen las ganancias, lo que a su vez retroalimenta el círculo virtuoso de crecimiento del consumo, de las inversiones y del producto. Los precios pueden mostrar una tendencia creciente, aunque esta no es una característica generalizable a todos los ciclos (Sachs y Larraín, 1994).

Crisis: marca el fin de la expansión y el comienzo de la contracción. La expectativa de ganancias crecientes que alimentaba a la inversión durante la expansión llega a su fin. Los proyectos de inversión que se realizaron hacia el final de la expansión no obtienen la rentabilidad esperada. Los capitales más pequeños comienzan a tener dificultades y comienzan a generalizarse las pérdidas y las quiebras que pueden afectar incluso a grandes capitales. La inversión se detiene y suele ubicarse en niveles en los cuales solo alcanza a cubrir la depreciación del capital, aunque también pueden darse casos de inversión neta negativa o desinversión. Se incrementa el desempleo y aumenta la capacidad ociosa. La crisis revierte la tendencia expansiva e inicia la contracción (Sachs y Larraín, 1994).

Recesión, contracción (depresión): durante esta fase se profundizan los efectos de la crisis. Desempleo creciente, salarios en baja, pérdidas crecientes y quiebras generalizadas, inversiones prácticamente nulas, problemas de realización que generan acumulación de inventarios. El círculo vicioso generado por todos estos factores retroalimenta la contracción, hasta llegar a 
un punto mínimo de actividad donde los factores que generan la contracción se agotan, debido a que el consumo presenta una variabilidad menor que el producto (Gaviola, 2011).

La fase de contracción o de depresión, también es llamada fase de receso o recesión. Los economistas suelen definir convencionalmente a la recesión por la caída en dos trimestres consecutivos del producto interno bruto de una economía. Por su parte el National Bureau Economic Research (NBER) define a la recesión como: "una caída significativa en la actividad económica que afecta a varios sectores dura por lo menos algunos meses $\mathrm{y}$ es generalmente visible en el producto interno bruto real, ingreso personal disponible real menos pagos de transferencias, empleo no agrícola, producción industrial y ventas de productos industriales y de comercios mayoristas y minoristas" (Business Cycle Dating Comitee, 2011).

Por su parte Gaviola (2011) explica que la caída del producto es detenida en el momento donde comienzan a aparecer oportunidades de inversión rentables, debido a que existe un consumo que es cada vez más inflexible a la disminución ante sucesivas caídas del producto. En el resto del trabajo se profundizará en este punto que es crucial para entender cuáles son las causas de la recuperación. También hay que decir que no todas las contracciones se transforman en depresiones. Así mismo, para el autor las depresiones son catástrofes sociales que generan miseria extrema, niveles grandes de desocupación, y gran cantidad de la población se ve sin la posibilidad de generar ingresos. En este sentido los dueños de las empresas ven diluirse a niveles muy bajos la valoración de sus activos, a su vez las compañías y los hogares endeudados no logran cumplir sus compromisos. En este contexto se produce una quiebra de la cadena de pagos, por lo que la demanda es incapaz de comprar el producto generado por la economía, lo cual obliga a la reducción permanente de este última. 
Se acumulan los stocks de mercaderías no vendidas, lo que hace que los pedidos de los comercios minoristas disminuyan, contrayendo de este modo la producción, y en consecuencia el empleo de la economía. Las depresiones son períodos de conmoción social donde no existen perspectivas optimistas, que en las economías de mercado suelen superarse con grandes ajustes, los cuales no están exentos de gran sufrimiento y penuria para la población, en especial para aquellos grupos de menores ingresos. Hasta aquí solo se han descripto algunos síntomas de cada una de las fases (Gaviola, 2011).

\section{Tipos de ciclos económicos}

En lo referente a los tipos de ciclos económicos, Gaviola (2011) señala que no existe un único tipo de ciclo económico y que el gran número de ciclos específicos que aparecen en los procesos económicos están claramente interrelacionados. En tal virtud, los tipos de ciclos más importantes que los estudiosos han identificado son los siguientes:

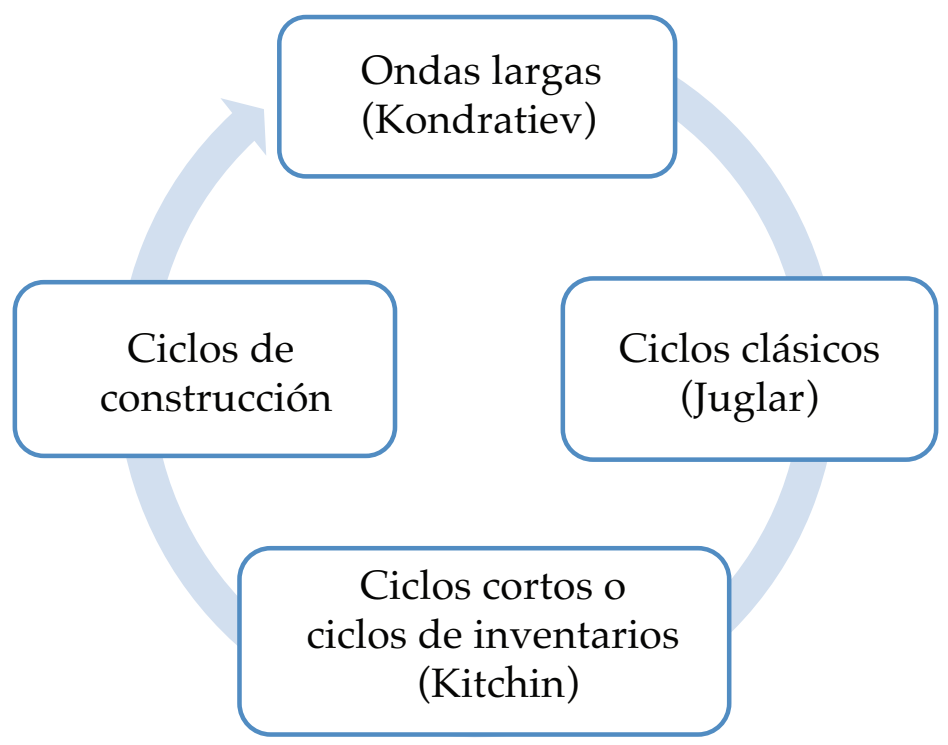

Figura 28. Tipos de ciclos económicos

Fuente: elaboración propia con base en Gaviola (2011). 


\section{Ciclo de ondas largas (Kondratiev)}

Los ciclos de ondas largas hacen referencia a movimientos cíclicos observables en series de precios y tasas de interés. A su vez cada onda consta de dos fases: una fase A de ascenso y otra fase B de depresión, cada una con una duración media de entre 25 y 30 años. Esta teoría conjuga la existencia de descubrimientos científicos importantes que impulsa de manera sustancial la demanda, la inversión y el empleo. En este sentido la aparición de nuevos productos que se hacen más accesibles cada vez para una cantidad mayor de la población empuja la fase expansiva del ciclo. La inversión se empieza detener cuando los mercados empiezan a saturarse y las empresas menos rentables empiezan a cerrar, generando la etapa recesiva del ciclo, a la espera de una nueva ola tecnológica (Giudice, 2010).

\section{Ciclos clásicos (Juglar)}

El término Juglar fue puesto en honor al médico francés Clement Juglar por el investigador Schumpeter, este tipo de ciclo identifica un ciclo de tres períodos: prosperidad, crisis y liquidación, que se guían continuamente en el mismo orden. Tuvo su análisis en el artículo "De las crisis comerciales, y de su retorno periódico" publicado en 1860, fue uno de los primeros en pasar de las crisis al análisis total de las fluctuaciones económicas (ciclos). Su mayor investigación la realizó en el siglo XIX en países como Estados Unidos, Francia e Inglaterra, teniendo ciclos de duración en promedio de 9 y 10 años. Este es uno de los ciclos que más se ha aprobado en la investigación empírica, lo contrario de los ciclos de ondas largas (Gaviola, 2011). 


\section{Ciclos cortos o ciclos de inventarios (Kitchin)}

Estos tipos de ciclos tienen en promedio una duración de 40 meses, y estaban asociados a las variaciones en los inventarios que poseen las empresas en su poder para poder satisfacer a la demanda.

\section{Ciclos de construcción}

Finalmente, los ciclos de construcción evidencian las fluctuaciones en la actividad de la construcción inmobiliaria con una duración media de entre 18 y 20 años.

\section{Teorías de los ciclos económicos}

\section{La escuela clásica}

La escuela clásica, llamada también escuela liberal, acuñó conceptos como libertad de producción y libertad de mercado. Los principales representantes en la evolución y desarrollo de la escuela fueron: Adam Smith, el principal aporte fue el libro sobre "La riqueza de las naciones, Jeremías Bentham con su tratado sobre "Defensa de la Usura", David Ricardo con "Principios de Economía Política", John Stuart Mill con Principios de Economía Política: Juan Bautista Say, "Tratado de Economía Política"; Thomas R. Malthus, "Ensayo sobre el principio de la población"; Federico Bastiat, "Armonías Económicas", "Ensayos sobre Economía Política"; Wesley Mitchell fue pionero en el estudio cuantitativo de los ciclos económicos.

Entre los principales supuestos de la visión clásica yacen los shocks con impactos positivos o negativos, cuyos precios se ajustan 
rápidamente para restablecer el equilibrio de empleo pleno, los mercados funcionan adecuadamente, el desempleo alto o la disminución del PIB son temporales, no se requiere intervención del gobierno. En la visión clásica, el cambio tecnológico es la influencia más importante que repercute sobre la demanda y la oferta agregada.

\section{$\underline{\text { Adam Smith (1723-1790) }}$}

Propuso varias teorías como la teoría del valor, con la finalidad de determinar la causa de las riquezas de las naciones, por lo tanto, estableció que el trabajo genera riqueza que se usa para bienes materiales. Además, estableció que el grado de productividad del trabajo de un país se da cuando produce más mercancías en el mismo tiempo, a diferencia de otros países. Según Smith las mercancías tienes dos clases de valor, considerando el valor de uso y el valor de cambio, por lo tanto, se mostraba el valor de una mercancía a través de la cantidad de trabajo incorporado (Smith, La riqueza de las naciones, 1776).

\section{Jeremías Bentham (1748-1832)}

Bentham estableció que la acción del Estado en los asuntos económicos era innecesaria. Respaldó y estimuló la competencia sin restricciones y el egoísmo ilustrado del individuo. Mediante los aportes a la economía que realizó Benthan se realizaron reformas sociales, cuidado de la salud pública, además de incidir por una distribución de la riqueza más equitativa (Bentham, 1965).

\section{$\underline{\text { David Ricardo (1772-1823) }}$}

Ricardo coincidía con Smith en establecer que el trabajo mide el valor no solamente de esa parte del precio que se deriva 
en salario, sino también de la que se descompone en renta y ganancia. Además, en su teoría de distribución estableció que la renta depende de la calidad de tierra, por lo tanto, se cultivan las tierras que estén más cerca de los centros de consumo o ciudades y sobre todo que sean más fértiles. Sin embargo, al aumentar la población, incrementa la necesidad de alimento, comenzando a cultivar las tierras de menor calidad, en las cuales se obtiene un menor rendimiento. David Ricardo establece el libre comercio sin barreras, pues de esta forma los países utilizarán mejor sus propios recursos. Considerando que cada país posee ventajas comparativas, por lo tanto, se podría producir y vender a los demás lo que estos no alcanzaban a cubrir y viceversa.

\section{Jhon Stuart Mill (1806-1873)}

Los estudios realizados por J.S. Mill se enfocaron en la producción donde recalca tres factores recursos naturales, trabajo y capital, admitió la existencia de ciertas leyes de la producción relacionada con los rendimientos decrecientes de la tierra. Consideró que se puede reducir la desigualdad de la distribución de la riqueza ya que los salarios, la renta de la tierra y las ganancias, están sometidos a la acción humana y a las decisiones de la sociedad.

\section{Juan Bautista Say (1767-1832)}

Los principales postulados económicos fueron la producción, distribución y consumo. Introdujo el término "entrepreneur" (empresario), para distinguir al prestamista de fondos de aquellos que combinan tierra, mano de obra y capital, para formar empresas de negocios. Expuso la ley de mercados, que indica que es la producción la que establece que la producción abre mercados a los productos, fortaleciendo la solidaridad inter-industrial y justifica el libre cambio. 


\section{Thomas R. Malthus (1776-1834)}

Estableció un principio de la población en 1798 que consistía en que la humanidad tiende a propagarse con mayor rapidez que el aumento de los precios de sustento. La propagación natural y libre de la población se efectúa en progresión genérica, mientras que el aumento de la producción de frutos de suelo se efectúa solo en progresión aritmética. Malthus consideró que se debían establecer políticas preventivas, como abstenerse de contraer matrimonio y de engendrar hijos, casarse a edad avanzada y por otra parte se fomentara la agricultura por todos los medios.

\section{Federico Bastiat (1801-1850)}

Estableció que el Estado no debe intervenir entre las empresas, ya que tendía a enriquecer a la minoría y defraudar a la mayoría, por lo tanto, las empresas debían tener una completa libertad.

\section{$\underline{\text { La escuela keynesiana }}$}

Los keynesianos creen que, si se deja la economía a su libre funcionamiento, esta no conseguirá llegar a un nivel de equilibrio de pleno empleo, y que por lo tanto para llegar a ese nivel, es necesario de la ayuda de la política fiscal y monetaria. El principal representante fue John Maynard Keynes con su obra Teoría General del Empleo, el interés y el dinero (1936), él para explicar la Gran Decadencia estableció ideas básicas (Snowdon \& Howard, 2005). Los mercados no funcionan bien siempre, existe una posibilidad que los precios no se ajustan para mantener un equilibrio, el gobierno debe llevar a cabo políticas monetarias y fiscales para aumentar el PIB durante una recesión. En la visión keynesiana, las expectativas es la causa que actúa sobre la demanda agregada 
y se basa en el instinto del conglomerado o lo que Keynes llamó el espíritu animal, en este sentido un pesimismo generalizado puede llevar a una caída de la demanda y por la tanto a una recesión económica.

Por el lado de la oferta estiman que el salario nominal en el corto plazo es rígido a la baja, y por lo tanto ante una brecha recesiva, no hay un mecanismo automático que permita la regulación y la economía se estancará en la depresión (Parkin, Esquivel, y Muñoz, 2007).

\section{John Maynard Keynes (1883-1946)}

Keynes se centró en el ciclo de crédito, en sus investigaciones tuvo influencia wickselliana estimando que la inversión depende del ingreso futuro que el empresario anticipa de la inversión corriente en comparación con la tasa de interés que debe pagar para financiar su producción, mientras que el ahorro es estimulado por una alta tasa de interés (Keynes, 1936).

Las ecuaciones que Keynes denomina fundamentales son las siguientes:

$\mathrm{P}=\mathrm{E} / \mathrm{O}+\left(\mathrm{I}^{\prime}-\mathrm{S}\right) / \mathrm{R}$ y $\pi=\mathrm{E} / \mathrm{O}+(\mathrm{I}-\mathrm{S}) / \mathrm{O}$

Donde:

$\mathrm{P}=$ nivel de precios de los bienes de consumo

$\mathrm{R}=$ producto real de bienes de consumo

$\mathrm{E}=$ ingreso monetario de la comunidad

$\mathrm{O}=$ producto real compuesto por bienes de consumo y de inversión $\mathrm{I}^{\prime}=$ ingreso ganado en las industrias productoras de bienes de inversión

I = valor de los nuevos bienes de inversión 
$\mathrm{S}=$ ahorro nominal

$\pi=$ nivel de precios del producto total

Según la descripción del tratado de Keynes, el ciclo se iniciará a partir de algún evento no monetario que estimule la inversión. De no mediar un aumento en el ahorro, la tasa natural de interés se elevará. La participación del sistema bancario en el financiamiento de la nueva inversión se traduce en el aumento de la cantidad de dinero circulante. El producto y los precios de los bienes de capital se elevarán. El empleo mejorará y el índice de precios al por mayor se elevará. Se acrecentará el gasto de los nuevos empleados, y por consiguiente el precio de los bienes de consumo y las ganancias de sus productores aumentarán (Keynes, 1936). En la Teoría General no aparecen descripciones detalladas de las fases del ciclo, a partir de un choque no monetario y considera que el ciclo económico es ocasionado por un cambio cíclico en la eficiencia marginal del capital, aunque complicado y con frecuencia agravado por cambios asociados en otras variables significativas de corto plazo del sistema económico (Keynes, 1936).

Keynes dice que la duración del ciclo económico dependerá de la duración de los bienes de capital de una época. Es decir, la eficiencia marginal del capital solo volverá a incrementarse cuando su escasez relativa aumente. La única forma de lograr esto es la contracción del stock actual de capital de una época. Esto solo sucede por medio de su desgaste (depreciación real), su obsolescencia o su decaimiento. En mercados altamente tecnológicos la obsolescencia será un factor muy importante, pero en otros mercados la variable que domine puede ser la depreciación real (Flores, 2015).

Este proceso puede volverse más lento si se toma en cuenta que una caída en la eficiencia marginal del capital lleva a una caída en 
el mercado accionario y que esto a su vez representaba un efecto negativo en la propensión a consumir. De esta manera el uso de los bienes de capital puede ser mucho menor que antes de la crisis y así reducirse el nivel de depreciación real. Este enunciado sobre la duración del ciclo económico puede ser a mi entender válido. Dada una población creciente niveles cada vez más bajos de stock de capital provocarán un aumento en su desempeño marginal. De esta forma el ciclo puede durar aproximadamente lo que el capital tarde en tornarse escaso otra vez (Flores, 2015).

\section{El enfoque de Slutsky y Frisch}

Estos autores se enfocaron en la naturaleza estocástica del ciclo, estableciendo que las economías siguen trayectorias de equilibrio a partir de las cuales pueden alejarse temporalmente debido a eventos aleatorios (Kalecki, 1937).

\section{$\underline{\text { Slutzky (1880-1948) }}$}

Slutzky (1937) realizó un análisis donde indica que los ciclos económicos no repiten ciclos anteriores ni en duración ni en amplitud. Frisch demostró el principio de aceleración estableciendo una analogía entre las fluctuaciones económicas y las ondas que la física estudiaba, por lo tanto, las fluctuaciones dependen del principio de aceleración, considerando el efecto sobre la inversión de las variaciones en el nivel de renta real o producción.

\section{Michal Kalecki (1899-1970)}

Michal Kalecki realizó contribuciones en la macro dinámica keynesiana, en enero de 1936 se publicó la Teoría General de 
la Ocupación, el Interés y el Dinero. Simultáneamente, Michal Kalecki había llegado a la misma solución. En una obra publicada en Polonia en 1933, Kalecki formulaba claramente el principio de la demanda efectiva en términos matemáticos, en la misma el sistema kaleckiano se expresa mediante un modelo lineal de naturaleza mixta incorporando términos diferenciales y términos en diferencias, Kalecki obtuvo fluctuaciones cíclicas constantes (Kalecki, 1937).

Según Kalecki el ciclo económico parte de conocer la decisión de invertir, y el rezago temporal entre la decisión de invertir y la instalación de las inversiones, a la vez la decisión de invertir está influenciada positivamente de los beneficios y negativamente de la acumulación de capital. La distribución del producto entre capitalistas y trabajadores es un tema central en la discusión kaleckiana del ciclo. El ingreso en la forma de salarios sería recibido por los trabajadores, los cuales lo consumirían en su totalidad; a su turno, el ingreso en la forma de beneficios sería recibido por los capitalistas, los cuales lo ahorrarían en su totalidad (Kalecki, 1937).

Por lo tanto, el ciclo económico es producido por la interrelación entre los pedidos de bienes de inversión, su producción, la entrega de los nuevos equipos para su instalación y el cambio de la acumulación de capital, la inversión considerada como el gasto de los capitalistas es la fuente de la prosperidad, y cada uno de sus aumentos mejora los negocios y estimula futuros incrementos de la inversión. Al mismo tiempo la inversión es una adición al equipo de capital, de modo que cada incremento de este llega a competir con las antiguas generaciones de dicho equipo. La tragedia de la inversión consiste en que ella atrae la crisis precisamente porque es útil (Kalecki, 1937). 


\section{Nicholas Kaldor (1908-1986)}

Nicholas Kaldor intentó mostrar las condiciones bajo las cuales el multiplicador keynesiano juntamente con la función de demanda de inversión producen el ciclo económico, descartó las funciones lineales de ahorro e inversión relacionado con el ingreso. Estableciendo que el equilibrio de los ciclos económicos es constante solo en el corto plazo, en el caso de largo plazo se generará una acumulación de capital y el crecimiento del ingreso real que provoca inestabilidad, por lo tanto, considera que el ciclo económico se genera endógenamente sin choques exógenos (Kaldor, 1961).

\section{$\underline{\text { Richard Goodwin (1913-1996) }}$}

Goodwin propone un modelo de ciclo económico análogo a los de Samuelson y Hicks, sin embargo, considera la interacción del multiplicador y el acelerador, la dificultad con el principio de aceleración es que supone que la acumulación de capital real se mantiene de acuerdo con la relación deseada con el producto, por lo tanto, en algún momento habrá un exceso de capital y en otro una deficiencia, así cuando la acumulación de capital es insuficiente, la tasa de inversión aumenta, y cuando es excesivo se produce desacumulación de capital (Goodwin, 1951).

\section{Sir Roy F. Harrod (1900-1978)}

El modelo de crecimiento propuesto por Harrod estudia las fluctuaciones en una tendencia ascendente, considerando que el ciclo comenzó a aparecer con la revolución industrial, donde dedujo que la expansión de la renta nacional es característica del sistema económico. 
Phillips (1958) estudió la relación empírica en donde describe la tasa de crecimiento de los salarios nominales como una función del desempleo:

$\mathrm{W}=\mathrm{f}(\mathrm{mn}-\mathrm{u})$

Si la inflación salarial puede ser una buena medida de la variación del nivel de precios, entonces:

$\pi=\mathrm{f}(\mathrm{un}-\mathrm{u})$

La Curva de Phillips

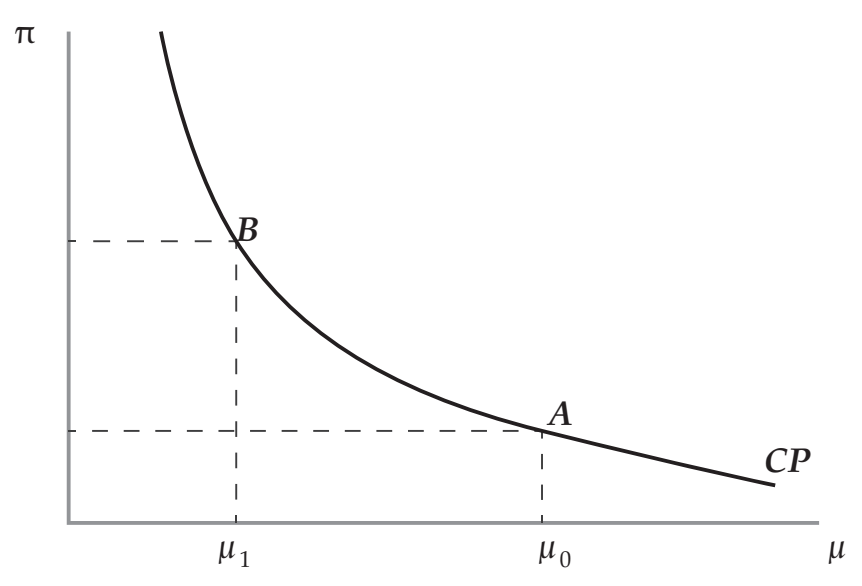

Figura 29. Curva de Phillips

Fuente: elaboración propia.

Demostró, aparentemente, una relación negativa y estable entre las tasas de empleo e inflación. Por lo tanto, estableció que, si los políticos deseaban disminuir la tasa de desempleo, el costo sería una tasa más alta de inflación. 


\section{$\underline{\text { Neoclásico keynesiano }}$}

John R. Hicks (1904-1989)

Hicks consideró que la economía keynesiana, a pesar de evidenciar las fluctuaciones económicas, ha dejado inexplicada el ciclo económico, ya que no se ha establecido el nivel de las fluctuaciones conforme a un modelo definido (Hicks, Contribution to the Theory of the Trade Cycle, 1949). Hicks corroboró que la inversión autónoma está ligada a una tasa de crecimiento exógena, así, los ciclos evolucionaron con trayectorias explosivas alrededor a la inversión autónoma, encontrándose con techos y pisos que controlan las fluctuaciones y evitan que sobrepasen ciertos límites (Hicks, Contribution to the Theory of the Trade Cycle, 1949).

\section{Paul A. Samuelson (1915-2009)}

Samuelson enfocó sus estudios entre el análisis del multiplicador y el principio de aceleración, partiendo de la función de consumo lineal como se indica a continuación:

$\mathrm{Ct}=\mathrm{CO}+\mathrm{cYt}-1$

Donde:

$\mathrm{C}=$ consumo total en el período $\mathrm{t}$

$\mathrm{co}=$ consumo autónomo

$\mathrm{C}=$ propensión a consumir

$\mathrm{Y}=$ ingreso.

El hacer depender a $C_{t}$ de $Y_{t-1}$ se conoce en la literatura macrodinámica con el nombre de rezago de Robertson. La función de inversión era de forma lineal $I_{t}=I_{o}+\beta\left(C_{t}-C_{t-1}\right)$, en donde $\beta$ es 
el parámetro del acelerador, que Samuelson al igual que Harrod llamó también relación. Entonces, el equilibrio macroeconómico sería:

$$
\begin{aligned}
& \mathrm{Y}_{\mathrm{t}}=\mathrm{C}_{\mathrm{t}}+\mathrm{I}_{\mathrm{t}}=\mathrm{c}_{\mathrm{o}}+\mathrm{I}_{\mathrm{o}}+\mathrm{c} \mathrm{Y}_{\mathrm{t}-1}+\beta\left(\mathrm{C}_{\mathrm{t}}-\mathrm{C}_{\mathrm{t}-1}\right) . \\
& \mathrm{C}_{\mathrm{t}}=\mathrm{c}_{\mathrm{o}}+\mathrm{c} \mathrm{Y}_{\mathrm{t}-1} \mathrm{y} \mathrm{C}_{\mathrm{t}-1}=\mathrm{c}_{\mathrm{o}}+\mathrm{cY} \mathrm{Y}_{\mathrm{t}-2}
\end{aligned}
$$

Se obtiene finalmente la siguiente ecuación en diferencias lineal de segundo orden:

$$
Y_{t}-(1+\beta) c Y_{t}+\beta c Y_{t-2}=c_{o}+I_{o}
$$

De la solución de este sistema se obtienen entre otros resultados que si $\mathrm{c}=1 / \beta$, las oscilaciones son constantes, que si $\mathrm{c}<1 / \beta$, las oscilaciones son amortiguadas, y que si $c>1 / \beta$, las oscilaciones son explosivas.

Por lo tanto, Samuelson obtuvo ciclos regulares al darse cuenta que el consumo en el período corriente dependía de un componente autónomo, y del ingreso en el período anterior. Igualmente, la inversión tenía un término autónomo, y otro que hacía depender la inversión en el período corriente de la diferencia entre el consumo corriente y el consumo en el período anterior. Esta diferencia entre los valores del consumo era la forma de incorporar el principio de aceleración.

Hicks empleó un modelo lineal que incorpora tanto el multiplicador como el acelerador. La función de consumo es la misma de Samuelson, pero la inversión es diferente. La función empleada fue la siguiente:

$I_{t}=I_{o}+\beta\left(Y_{t-1}-Y_{t-2}\right)$ 
Por lo tanto, el equilibrio macroeconómico estaría dado por:

$$
\mathrm{Y}_{\mathrm{t}}=\mathrm{C}_{\mathrm{t}}+\mathrm{I}_{\mathrm{t}}=\mathrm{c}_{\mathrm{o}}+\mathrm{I}_{\mathrm{o}}+\mathrm{c} \mathrm{Y}_{\mathrm{t}-1}+\beta\left(\mathrm{Y}_{\mathrm{t}-1}-\mathrm{Y}_{\mathrm{t}-2}\right)
$$

Se obtiene una ecuación lineal en diferencias de segundo orden,

$$
Y_{t}-(c+\beta) Y_{t-1}+\beta Y_{t-2}=\left(c_{o}+I_{o}\right)
$$

De la solución de este sistema se deriva que cuando $\beta=1$ para cualquier valor de $c$, se producen oscilaciones constantes, y para cualquier valor de $\beta$ por encima o por debajo de 1 , oscilaciones explosivas o amortiguadas.

\section{Lawrence Robert Klein (1920-2013)}

Según Klein (1983) entre los años treinta a los sesenta se acuñó la expresión el ciclo económico ha muerto, durante ese período se denominó política económica, siendo el manejo de la política monetaria y fiscal, y así reducir al mínimo las fluctuaciones económicas. Los estudios se enfocaron en los niveles de producto y empleo y así dieron lugar a representaciones agregadas de la economía, permitiendo analizar interacciones entre variables monetarias y variables reales, el caso más relevante fue el modelo IS-LM.

\section{$\underline{\text { Robert M. Solow (1924) }}$}

Solow propuso un modelo de crecimiento de coeficientes estableciendo que las economías estarían sujetas a la alternación de prolongados períodos de desempleo y largos períodos de oferta laboral insuficiente (Solow, 1979). 


\section{Monetarismo}

\section{Milton Friedman (1912-2006)}

Friedman (1968) estableció que las fluctuaciones de la oferta monetaria son responsables de la mayoría de las grandes fluctuaciones de la economía. Estableció la teoría cuantitativa del dinero discrepando de Keynes, por lo tanto, su teoría se fundamentó en que: la demanda de dinero es estable, entonces la velocidad de dinero $(\mathrm{V})$ es estable:

$\mathrm{MV}=\mathrm{PY}$

Los cambios de la cantidad de dinero (M) es estable. Friedman propuso que la oferta monetaria crece lentamente y a una tasa constante. Friedman fue uno de los principales detractores de la curva de Phillips ya que no consideraba valores reales, por lo tanto, demostró que la curva puede desplazarse cuando las expectativas de inflación varían. Al existir un desplazamiento de la curva la relación entre las tasas de inflación y desempleo no es estable. Presentando la idea que hay una tasa natural de desempleo que tiende a largo plazo.

\section{La nueva economía clásica}

\section{$\underline{\text { Leon Walras (1834-1910) }}$}

Considerado por Joseph Schumpeter como el economista más importante de todos los tiempos, estableció la teoría de la utilidad marginal, siendo el primer economista que desarrolló un sistema multi-ecuacional del equilibrio económico general (Walras, 1898). 
Los modelos propuestos por Walras tuvieron algunas implicaciones:

- La política monetaria es ineficaz, ya que un cambio inesperado afecta a las variables temporalmente.

- Las reglas establecidas en la política monetaria influyen positivamente en el modelo

- La disminución de los impuestos, financiada por la emisión de bonos no afecta el consumo agregado.

- El PIB incrementa si el gobierno aumenta los incentivos de trabajo e inversión.

- Los parámetros de los modelos varían si las políticas cambian, por lo que no se debe emplear para la evaluación de políticas alternativas.

\section{Ciclos económicos reales}

De Gregorio (2007) indica sobre los modelos de ciclo de negocio real, mejor conocidos por sus siglas en inglés como RBC por real business cycle. Han sido estudiados por autores como Kydland y Prescott (1982), Long y Plosser (1983), King, Plosser y Rebelo (1988) y Prescott (1986), y su impacto en la investigación económica ha sido tan importante que es una de las razones por las cuales Finn Kydland y Edward Prescott obtuvieron el premio Nobel de Economía el 2004.

Los ciclos económicos reales se basan en:

- Las ofertas de los factores de producción (trabajo, capital) y tecnología determinan la tendencia a largo plazo de la economía.

- Las variaciones de la demanda agregada determinan las desviaciones temporales de la tendencia, es decir los ciclos económicos. 
- $\quad$ Si los cambios del PIB real son permanentes, los cambios de la oferta monetaria no puede ser las causas porque, a largo plazo, el dinero es neutral (no tiene efectos reales).

El modelo RBC supone la existencia de shocks que perturba la economía y causa fluctuaciones. Uno de los shocks más estudiados es el de productividad, seguido por shocks fiscales, es decir, cambios en el gasto público o impuestos, shocks a las preferencias, shocks de costos, como el de fluctuaciones en el precio del petróleo. Por lo tanto, el modelo RBC emplea shocks para explicar las fluctuaciones del PIB, por lo tanto, su base está en el espíritu de los modelos de pleno empleo no basta con asumir un shock, ya que estos no son de una magnitud suficiente dadas las fluctuaciones y la dinámica de los agregados macroeconómicos que se acostumbra observar en la realidad. Por lo tanto, se emplean mecanismos de propagación, a través de los cuales los shocks se difunden y amplifican como son los RBC.

La primera complicación es la definición de la tendencia de las variables y comparar esta tendencia con el dato efectivo para definir el componente cíclico o desviación de la tendencia. Existen métodos para estimar las tendencias, como es el filtro de Hodrick y Prescott, que se encarga de suavizar los datos para separar la tendencia del ciclo. En todas las economías, el componente más volátil del ciclo económico, por el lado de la demanda, es la inversión, la que es más de tres veces más variable que el producto. Si bien no se presenta la volatilidad relativa de la política fiscal, esta es mucho menor que a la inversión.

\section{Modelo básico del ciclo económico real}

Según De Gregorio (2007) el análisis del modelo básico del ciclo económico real se expresa desde dos puntos de vista Hogares y Empresas como se describe: 


\section{$\underline{\text { Hogares }}$}

Los hogares tienen un horizonte infinito y deciden su consumo de bienes $\left(C_{t}\right)$ y ocio $(\mathrm{Zt})$ de manera de maximizar el valor esperado de su utilidad intertemporal, donde su tasa de descuento es o. Los hogares tienen un solo individuo y este dispone de una unidad de tiempo, de manera que su oferta de trabajo, $\mathrm{L}_{\mathrm{t}^{\prime}}$ será $1-\mathrm{Z}_{\mathrm{t}}$. $\max E_{t} \sum_{\delta=0}^{\infty} \frac{1}{(1+\rho)^{\delta}} u\left(C_{t+\delta}, 1-L_{t+\delta}\right)$

Los consumidores reciben un salario $\mathrm{w}_{\mathrm{t}}$ por su trabajo, $\mathrm{y}$ una tasa de retorno $r_{t}$ por sus ahorros $\left(A_{t}\right)$ a principios del período $t$. Los hogares también pagan impuestos $\mathrm{T}_{\mathrm{t}}$ de suma alzada en cada período. En consecuencia, su restricción en cada período será:

$$
\left(1+r_{t}\right) A_{t}+w_{t} L_{t}=C_{t}+A_{t+1}+\tau_{t}
$$

Por lo tanto, el lagrangiano de este problema será:

$$
\begin{aligned}
& L_{t}=E_{t} \sum_{\delta=0}^{\infty} \frac{1}{\left(1+\rho^{\delta}\right)} u\left(C_{t+\delta}, 1-L_{t+\delta}\right)+\lambda_{t+8}\left[C_{t+8}+A_{t+8+1}+\right. \\
& \tau_{t+8}-\left(1+r_{t+8}\right) A_{t+8}-w_{t+\delta} L_{t+\delta}
\end{aligned}
$$

Las condiciones óptimas intratemporales para elegir consumo y trabajo son:

$$
\begin{aligned}
& u_{C}\left(C_{t}, 1-L_{t}\right)=\lambda_{t} \\
& u_{L}\left(C_{t}, 1-L_{t}\right)=-\lambda_{t} w_{t}
\end{aligned}
$$

Al eliminar $\lambda$ en las ecuaciones anteriores, tenemos la tradicional condición entre empleo y consumo:

$$
u_{C}\left(C_{t}, 1-L_{t}\right)=\frac{-u_{L}\left(C_{t}, 1-L_{t}\right)}{w_{t}}
$$


La decisión óptima de consumo intertemporal se resuelve examinando los efectos de mover el consumo de un período a otro. Por lo tanto, sacrificar una unidad de consumo significa menor utilidad por $u_{C}\left(C_{t^{\prime}} 1-L_{t}\right)$. El siguiente período generará $1+r_{t+1}$ unidades del bien que produce un marginal de $u_{C}\left(C_{t+1}, 1\right.$ $\left.-\mathrm{L}_{\mathrm{t}+1}\right)$, descontada por $1+\mathrm{Q}$. Igualando las utilidades marginales debidamente valoradas en ambos períodos se establece que, el óptimo está dado por:

$u_{C}\left(C_{t}, 1-L_{t}\right)=E_{t} \frac{1+r_{t+1}}{1+\rho} u_{C}\left(C_{t+1}, 1-L_{t+1}\right)$

Al realizar esta relación se obtiene la pendiente en función al consumo, y una que se reemplaza en la restricción presupuestaria, debería dar el nivel del consumo en cada período.

\section{$\underline{\text { Empresas }}$}

Las empresas producen bienes arrendando trabajo y capital, de acuerdo con la siguiente función de producción:

$\overline{Y_{t}}=a_{t} F\left(K_{t}, L_{t}\right)$

La productividad total de los factores es denotada por a, y $\mathrm{K}$ y L son capital y trabajo, respectivamente. Considerando que se ha asumido que el número de hogares es 1 , de manera que la oferta total de trabajo es igual a la oferta de trabajo del hogar representativo. Por lo tanto, las variables son per cápita. El capital se deprecia a una tasa $\delta$ y hay perfecta competencia en todos los mercados. En consecuencia, las empresas arrendarán capital y trabajo de manera que su productividad marginal iguale a su precio, es decir:

$$
\frac{w_{t}=a_{t} F_{L}\left(K_{t}, L_{t}\right)}{r_{t}=a_{t} F_{K}\left(K_{t} L_{t}\right)-\delta}
$$


La última ecuación plantea que el capital óptimo es el que iguala su productividad marginal $\left(\mathrm{af}_{\mathrm{K}}\right)$ con su costo de uso, que es costo alternativo de los fondos ( $r$ ) más la depreciación ( $\delta$ ). Para un equilibrio general se deben cumplir tres condiciones:

Los hogares maximizan su utilidad.

Las empresas maximizan utilidades.

Todos los mercados están en equilibrio.

Estas condiciones establecen sobre las curvas de oferta y demanda y el equilibrio general ocurre cuando las ofertas igualan a las demandas. El equilibrio de este modelo consiste en que las empresas y los hogares maximizan sus utilidades y los mercados están en equilibrio. El único activo es el capital por lo que $\mathrm{A}_{\mathrm{t}}=\mathrm{K}_{\mathrm{t}}$. Al reemplazar en la restricción presupuestaria se tiene que:

$\overline{Y_{t}+(1-\delta) K_{t}=C_{t}+K_{t+1}+\tau_{t}}$

Al cumplirse la equivalencia ricardiana, se asume que $G_{t}=T_{t}$. Por último, la inversión $\mathrm{I}_{t}$ corresponde a la acumulación neta de capital, entonces:

$\overline{K_{t+1}-(1-\delta) K_{t}}$

Por lo tanto, la condición de equilibrio de mercado es:

$\overline{Y_{t}=C_{t}+I_{t}+G_{t}}$

Siendo la igualdad entre la oferta de bienes y el gasto, representándola de mejor forma así:

$$
a_{t} F\left(K_{t}, L_{t}\right)=C_{t}+K_{t+1}-(1-\delta) K_{t}+G_{t}
$$


Se ha llegado a este punto gracias a la maximización de las utilidades de los hogares y de las empresas, representando un equilibrio de mercados de bienes y capitales. En esta investigación se va a basar en hechos estilizados de los ciclos económicos, por lo tanto, se analizará a través de la variabilidad, la dirección, la persistencia y la temporalidad del PIB (Snowdon \& Howard, 2005). Según Jones y Romer (2010), los hechos estilizados analizan factores como la renta nacional, capital físico, capital humano, considerando que los hechos estilizados fueron propuestos por Kaldor (1961).

\section{Fundamentos teóricos de los modelos RBC}

Los modelos RBC cuentan con supuestos que se enfocan en el tipo de economía, mercado y en los agentes que participan en el modelo.

Los modelos RBC suponen una economía cerrada, implicando que la inversión sea igual al ahorro, sin embargo, se ha extendido el modelo a una economía abierta (Mendoza, 1991).

Los modelos de factores y bienes finales son de competencia perfecta, sin embargo se han realizado evaluaciones del modelo RBC bajo el supuesto de competencia monopolística en el mercado de bienes.

Se asume dos agentes económicos familias y empresas, si se considera el gobierno se lo hace por medio de su restricción presupuestaria, además la autoridad monetaria es expresada por su restricción presupuestaria y por la política monetaria.

La única fuente de incertidumbre proviene de la oferta, con choque de productividad. 
El único bien producido es utilizado para el consumo y la inversión.

\section{La escuela austriaca}

La escuela austriaca es una de las tres escuelas que impulsaron la revolución marginalista a finales del siglo XIX. En esta prevalecían el pensamiento de las libertades civiles, la igualdad ante la ley, el dinero sano y la libertad de comercio. Entre los representantes más importantes figuran: Carl Menger (1840-1921), Eugen Von Böhm-Bawerk (1851-1914), Ludwig von Mises (1881-1973), Friedrich A. von Hayek (1899-1992). Esta escuela manifiesta que el ciclo económico siempre empieza por una expansión de crédito generado por un aumento de la oferta monetaria, que no tiene respaldo de oro ni un ahorro previo. Por lo general este evento se desarrolla cuando los bancos centrales tienen una emisión alta de dinero y cuando bajan los tipos de interés (Mises, 2005).

En ese contexto, Friedrich Von Hayek señala que el origen de las crisis económicas era la expansión artificial del crédito (dinero endógeno) y la manipulación de las tasas de interés, porque "distorsionan los incentivos de inversión y la asignación de recursos en la economía" (Economía y Desarrollo, 2020). Para evitar una posible quiebra del sistema financiero, los economistas de la escuela austriaca proponen como solución un coeficiente legal de caja del $100 \%$ para responder a la demanda del valor de los depósitos de los clientes en cualquier momento, al evitarse una posible quiebra del sistema financiero ya que las personas podrían acceder al valor de sus depósitos en cualquier momento, se evitarían las recesiones económicas y, también los ciclos económicos (Economía y Desarrollo, 2020). 
Uno de los precursores de esta idea fue Ludwig Von Mises quien afirmaba que "no debería haber más expansión crediticia y que en el futuro no se introducirá ningún crédito adicional a menos que exista una cobertura del $100 \%$ en dinero". Hayek apoyaba esta propuesta puesto que la veía como la forma de eliminar los ciclos económicos y las crisis causadas por los mismos (Economía y Desarrollo, 2020).

\section{Los ciclos económicos del Ecuador}

En el Ecuador, se estableció un sistema de indicadores de los ciclos económicos para inferir el comportamiento de acuerdo con la fase por la cual está atravesando la economía. Se constatan seis ciclos de crecimiento completos con una duración promedio de 73 meses. Cada ciclo consta de 4 fases: desaceleración sobre tendencia, desaceleración bajo tendencia, recuperación y expansión. Estos se analizan desde el primer trimestre de 1993 hasta el primer trimestre de 2017 (Banco Central del Ecuador, 2017).

El ciclo del crecimiento del producto interno bruto es de gran importancia como un indicador que determina la etapa en que se encuentra el ciclo económico. Ecuador en los últimos años ha sufrido procesos económicos transversales en los cuales se puede mencionar el período de recesión por el feriado bancario que sucedió en el 2000. En el año 2004 la economía presentó un crecimiento con respecto a los años anteriores, posteriormente en los años 2008 y 2009 se dio una crisis la cual tuvo su origen en Estados Unidos, por la denominada "Burbuja Inmobiliaria, posteriormente, en el año 2016, se presenta un período recesivo en la economía y que fue dado por algunos factores entre los que se destacan: la caída de los precios del petróleo a nivel internacional, el terremoto del 16 de abril del mismo año y el mal manejo en materia de política económica (Cuero, 2019, pág. 16). 
En los años 2017 y 2018, el país presenta un crecimiento del 2,37\% y 1,29\%, debido a la variación positiva del gasto de consumo final de los hogares en $4,9 \%$ y $2,7 \%$, y el gasto de consumo final del gobierno con una variación del 3,8\% y 2,9\% respectivamente (EFE, 2018). En el año 2019, se muestra un decrecimiento de la economía 0,05\% esto debido al ajuste del tamaño del Estado, a los bajos precios del petróleo y el paro nacional de octubre. A continuación, se muestra el desempeño del producto interno bruto de Ecuador.

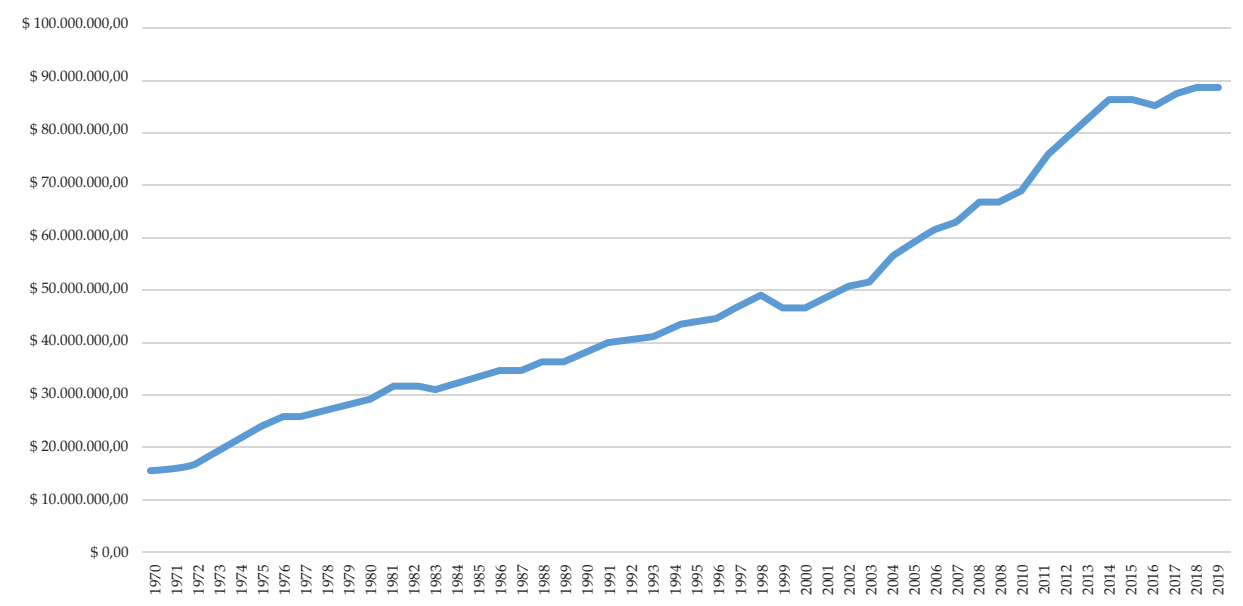

Figura 30. Producto Interno Bruto de Ecuador (precios constantes). Período 19702019

Fuente: elaboración propia con base en Banco Mundial (2019).

Pacheco(2006), mediante la aplicación del filtro Hodrick Prescotten la economía ecuatoriana, establece las interacciones de los patrones cíclicos con la política fiscal. Los resultados que obtiene muestran una relación pro-cíclica del sector fiscal, específicamente del gasto total, la inversión pública y las remuneraciones del sector público con las fluctuaciones de la economía ecuatoriana. Sin embargo, la inversión pública es utilizada como variable de ajuste, por lo que los niveles de endeudamiento muestran un patrón contra-cíclico. Finalmente, se menciona que los multiplicadores fiscales (gasto) 
son fundamentales, puesto que generan efectos potenciales para estabilizar el ciclo económico en el país. Orellana (2011), mediante la metodología de Kydland y Prescott para describir los hechos estilizados, menciona que las exportaciones presentan un patrón pro-cíclico a la economía ecuatoriana y dos veces más volátil que el PIB. Adicionalmente indica que esta presenta un movimiento anticipado al ciclo, confirmando el papel protagónico del sector externo para países en vías de desarrollado. 


\section{Conclusiones}

Ninguna economía está exenta de sufrir fluctuaciones que estén alineadas a los ciclos económicos, y tampoco no hay un consenso sobre qué tipo de shocks de oferta o demanda son los causantes principales de estos desequilibrios, ya que las economías difieren en sus dimensiones fundamentales. Lo que afecta en un país, tal vez no sea determinante en otro, y lo que es bueno en un tiempo, no lo sea en otro momento.

Existen algunas teorías que explican el comportamiento de los ciclos económicos. La escuela clásica se basó en la libertad de producción y libertad del mercado. Se sostiene que los cambios en los precios relativos, especialmente en el salario real, es el mecanismo de optimización de la economía. En la escuela keynesiana el mercado laboral tarda en equilibrarse, ya que el salario nominal no se ajusta rápidamente a los cambios de la dinámica económica. Los neokeynesianos han ampliado su visión indicando que los ciclos económicos podrían darse no solo por la demanda sino también por la oferta agregada y la nueva economía clásica que considera que los shocks económicos se relacionan con la inestabilidad de las políticas monetarias, ya que un cambio inesperado afecta a las variables temporalmente.

Los modelos de los ciclos reales de negocios indican que los choques y perturbaciones de la oferta son la causa de las fluctuaciones económicas, siendo procedentes del desarrollo tecnológico que manifiestan cambios importantes en el incremento de la productividad, lo que conlleva a que las empresas y los trabajadores se adhieran a los cambios efectuados. Los choques de la oferta están fuera de control de la autoridad económica es decir que los ajustes que se dan recaen específicamente a las empresas y trabajadores, la política económica poco o nada puede hacer para 
estabilizar un ciclo de origen real. La escuela austriaca señala que el ciclo económico siempre empieza por una expansión de crédito generado por un aumento de la oferta monetaria, que no tiene respaldo de oro ni un ahorro previo. Por lo general, este evento se desarrolla cuando los bancos centrales tienen una emisión alta de dinero y cuando bajan los tipos de interés.

En Ecuador se constatan seis ciclos de crecimiento completos con una duración promedio de 73 meses. El ciclo del crecimiento del PIB es de gran importancia como un indicador que determina la etapa en que se encuentra el ciclo económico, se establece las interacciones de los patrones cíclicos con la política fiscal. Los resultados muestran una relación pro-cíclica del sector fiscal, específicamente del gasto total, la inversión pública y las remuneraciones del sector público. La actividad económica sucumbe ante shocks que pueden ser endógenos o exógenos de demanda o de oferta y que las causales difieren en espacio y tiempo por la estructura económica de cada economía, y la política económica constituye la herramienta que busca minimizar estos efectos en el corto plazo.

Como conclusión general la escuela clásica explica que el cambio tecnológico es la influencia más importante que repercute sobre la demanda y la oferta agregada. Por otra parte, la escuela keynesiana considera que, si se deja la economía a su libre funcionamiento, esta no conseguirá llegar a un nivel de equilibrio de pleno empleo, y que por lo tanto para llegar a ese nivel, es necesario de la ayuda de la política fiscal y monetaria, en cuanto al enfoque neoclásico keynesiano, se analiza el comportamiento del flujo de capitales y la desviación del equilibrio de pleno empleo. Por otra parte, para los monetaristas las fluctuaciones de la oferta monetaria son responsables de la mayoría de las grandes fluctuaciones de la economía. En la nueva economía clásica también han estudiado 
los ciclos económicos, y basándose en las expectativas racionales. Como resultado surge la teoría de los ciclos reales como una visión alternativa a la keynesiana. Finalmente, la escuela austriaca señala que el ciclo económico siempre empieza por una expansión de crédito generado por un aumento de la oferta monetaria. 


\section{Referencias}

Avella, M., \& Fergusson, L. (2003). El ciclo económico Enfoques e ilustraciones Los ciclos económicos de Estados Unidos y Colombia. Universidad de los Andes, 1-74.

Banco Central del Ecuador. (2017). Resultado del ciclo económco en el Ecuador. Obtenido de https://contenido.bce.fin. ec/ documentos / Estadisticas / SectorReal / Previsiones / IDEAC/IndiceCE.htm

Banco Mundial. (2019). Producto Interno Bruto a precios constantes. Obtenido de https://datos.bancomundial.org/indicator/ ny.gdp.mktp.kd

Bentham, J. (1965). The Philosophy of Economic Science-The Psychology of Economic Man. The Royal Economic Society. Obtenido de https: / / journals.openedition.org/oeconomia / 2249

Burns, A., \& Mitchell, W. (1946). Measuring Business Cycles. Massachusetts. Obtenido de https://econpapers.repec. org/bookchap/nbrnberbk/burn46-1.htm

Business Cycle Dating Comitee. Comunicado del 21 de octubre de 2001. National Bureau Economic Research (NBER), Washington.

Cuero, V. (2019). Ciclo económico y crédito: el caso de Ecuador en el período 2000-2017. (Tesis de Grado). Riobamba, Chimborazo, Ecuador.

De Gregorio, J. (2007). Macroeconomía Teoría y Políticas. Santiago de Chile: Pearson-Educación.

Economía y Desarrollo. (2020, 07 de Julio). La propuesta de la ESCUELA AUSTRIACA para eliminar los ciclos económicos. México. https://www.youtube.com/ watch? $\mathrm{v}=\mathrm{r} 6 \mathrm{iSy}$ odauN $4 \& \mathrm{t}=1 \mathrm{~s}$ 
EFE. (2018). La Economía de Ecuador creció 3\% en 2017. Portafolio. Obtenido de: https://www.portafolio.co/internacional/ crecimiento-economia-de-ecuador-en-2017-515670

Flores, C. R. (2015). Los Ciclos Económicos. Universidad Francisco Marroquin, 13.

Friedman, M. (1968). The Role of Monetary Policy. American Economic Review, 1-17.

Galindo, H., y Montecinos, A. (2017). Macrodinámica Modelos de Ciclos Económicos Reales. Arizona: Arizona State University. Gaviola, S. (2011). Capítulo I: Los ciclos económicos. En Salarios reales y Ciclos Económicos en Estados Unidos 1970-2001. Argentina: Universidad Nacional de Mar del Plata. Obtenido de: http:/ / www.academia.edu/450747/Los_ ciclos_econ\%C3\%B3micos

Giudice, V. (2010). Teorías de los Ciclos Económicos. Instituto De Investigaciones Económicas, Universidad San Marcos.

Goodwin, R. (1951). The Nonlinear Accelerator and the Persistence of Business Cycles. Econometrica, 1-17. https: / / doi.org/10.2307/1907905

Hicks, J. (1949). Contribution to the Theory of the Trade Cycle. Canadian Economics Association, XVII(3), 394-406. doi:https: / / doi.org / 10.2307 / 137700

Jones, C., \& Romer, P. (2010). “The New Kaldor Facts: Ideas, Institutions, Ppopulatios, and Human Capital". American Economic Journal: Macroeconomics, Vol 2, 224-245.

Kaldor, N. (1961). "Capital Accumulation and Economic Growth". The Theory of Capital (editado por FA Lutz y DC Hague), Macmillan, Londres, págs. 177-222.

Kalecki, M. (1937). A Theory of the Business Cycle. Review of Economic Studies, 4, 77-97. 
Keynes, J. (1936). La teoría general de la ocupación, el interés y el dinero. Reino Unido: Palgrave Macmillan.

King, R., Plosser, C. \& Rebelo, S. (1988). "Production, Growth, and Business Cycles: II. New Directions,". Journal of Monetary Economics, 21,309-42.

Klein, Lawrence R. (1983). La economía de la oferta y la demanda. Baltimore, Maryland: Prensa de la Universidad Johns Hopkins

Kydland, F. \& Prescott, E. (1982). Time to Build and Aggregate. Econometrica 50, 1345-1370.

Llorca, A. y Masó, F. (2016). La Inversión y el Ciclo Económico. Alicante: Universitas Lvcentina.

Long, J., \& Plosser, C. (1983.). Real Business Cycles. Journal of Political 91, 39-69.

Mendoza, E. (1991). Real Business Cycles in a Small Open Economy. American Economic Review, 81(4), 797-818.

Mises, L. (2005). La Teoria "Austriaca" del Ciclo Economico. Buenos Aires: Revista Libertas XII.

Orellana, M. (2011). Hechos estilizados del ciclo económico de Ecuador: 1990-2009. Revista de Ciencias Sociales y Humanas(15), 53-84.

Pacheco, D. (2006). Ecuador: Ciclo Económico y Política Fiscal. Obtenido de: https://contenido.bce.fin.ec/documentos/ PublicacionesNotas / Catalogo / Cuestiones / XXII-III01PACHECO.pdf

Parkin, M., Esquivel, G., y Muñoz, M. (2007). Macroeconomía, versión para Latinoamérica. México: Pearson.

Phillips, W. (1958). The Relationship between Unemployment and the Rate of Change of Money Wages in the United 
Kingdom 1861-1957. Economica, 25(100), 283-299. https:// doi.org/10.2307/ 2550759

Prescott, E. (1986). Theory Ahead of Business-Cycle Measurement. Carnegie-Rochester Conference Series on Public Policy 25 (págs. 11-44). Carnegie-Rochester.

Ramírez, F. (2010). Shocks Externos y Fluctuaciones Macroeconómicas en una Economía Pequeña y Abierta: Evidencia de la República Dominicana para el Período 1998 - 2008. Ciclos Económicos y Variables no observables, 4(1), 195278.

Resico, M.(2011). Introducción a la Economía Social de Mercado. Ciclos Económicos y Política Macroeconómica. Buenos Aires: Conrad Adenauer Stiftung.. Obtenido de: https://www. kas.de / c / document_library / get_file?uuid=22412104f255-886e-178f-8b32bf5bce06\&groupId $=252038$

Sachs, J. y Larraín, F. (1994). Macroeconomía en la economía global. Buenos Aires: PEARSON EDUCATION.

Schumpeter, J. (1939). Análisis del cambio económico. 17(4), 2-10. Obtenido de: https:/ / periferiaactiva.files.wordpress. com/2016/06/schump-cambio.pdf

Schumpeter, J. (1976). Capitalism, Socialism and Democracy. Routledge. 1-460. Obtenido de https://eet.pixel-online.org/ files/etranslation/original/Schumpeter,\%20Capitalism, \%20 Socialism\%20and\%20Democracy.pdf

Sevilla, A. (2015, 28 de Mayo). Ciclo económico. Obtenido de Economipedia: https://economipedia.com/definiciones / ciclo-economico.html

Slutzky, E. (1937). The summation of random cuases as the source of cyclic processes. Econometrica, 5(2), 105-146. 
Smith, A. (1776). La riqueza de las naciones. Londres. Obtenido de: https: / / www.marxists.org/espanol/smith_adam/1776/ riqueza/smith-tomo1.pdf

Snowdon, B., \& Howard, V. (2005). Modern Macroeconomics Its Origins, Development and Current State. Massachusetts: Edward Elgar Publishing. Inc.

Solow, R. (1979). Another Possible Source of Wage Stickiness. Journal of Macroeconomics Vol. 1, 79-82.

Tobón, A. (2008). Los precios en la nueva síntesis neoclásicakeynesiana en macroeconomía. Lecturas de economía, 201 220.

Walras, L. (1898). Theory of the Production of Social Wealth. Studies in Applied Economics Vol, 1, 1-417. 


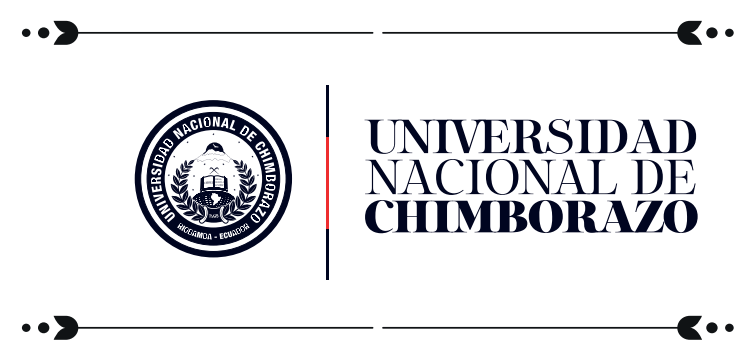

Gestión del Conocimiento y Propiedad Intelectual

INFORMACIÓN, GESTIÓN Y ECONOMÍA, Reflexiones desde la experiencia ecuatoriana; se publicó en el mes de enero de 2022 en la Universidad Nacional de Chimborazo. 


\title{
INFORMACIÓN, GESTIÓN Y ECONOMÍA
}

\author{
Reflexiones desde la \\ experiencia ecuatoriana
}

La información es un elemento fundamental en la economía; ya sea en el ejercicio de lo público o de lo privado, desde lo macro hasta la circunstancia más cotidiana, la información está presente. La información y el acceso a la misma juega un papel cada vez más importante para el funcionamiento de las empresas, el mercado y el gobierno. La información es en sí una fuerza productiva y la base de todas las decisiones. Por tanto, su acceso y gestión es un factor fundamental. Es un derecho y un componente trascendental del desarrollo.

La información es un activo que actualmente se ha convertido en un elemento clave en la estrategia competitiva de países y empresas. En las sociedades actuales, solo perviven las organizaciones que gestionan su información y crean nuevos conocimientos y los gestionan adecuadamente. Es decir, aquellas que reconocen a la información como un recurso indispensable para ampliar su competitividad y aumentar la calidad de los servicios que prestan a ciudadanos y clientes. Las organizaciones serán más valoradas en la medida que tengan una mayor capacidad para gestionar su información, y que esto sea la base para su competitividad y su conexión con clientes y ciudadanos.

Ahora bien, la información que surge de datos u observaciones debe ser interpretada para que surja el conocimiento. Por tanto, es necesaria una capacidad de interpretar. La información son datos dentro de un contexto y el conocimiento es información sujeta a la reflexión y a otras prácticas que provean un entendimiento más profundo (Erickson y Rothberg, 2014).
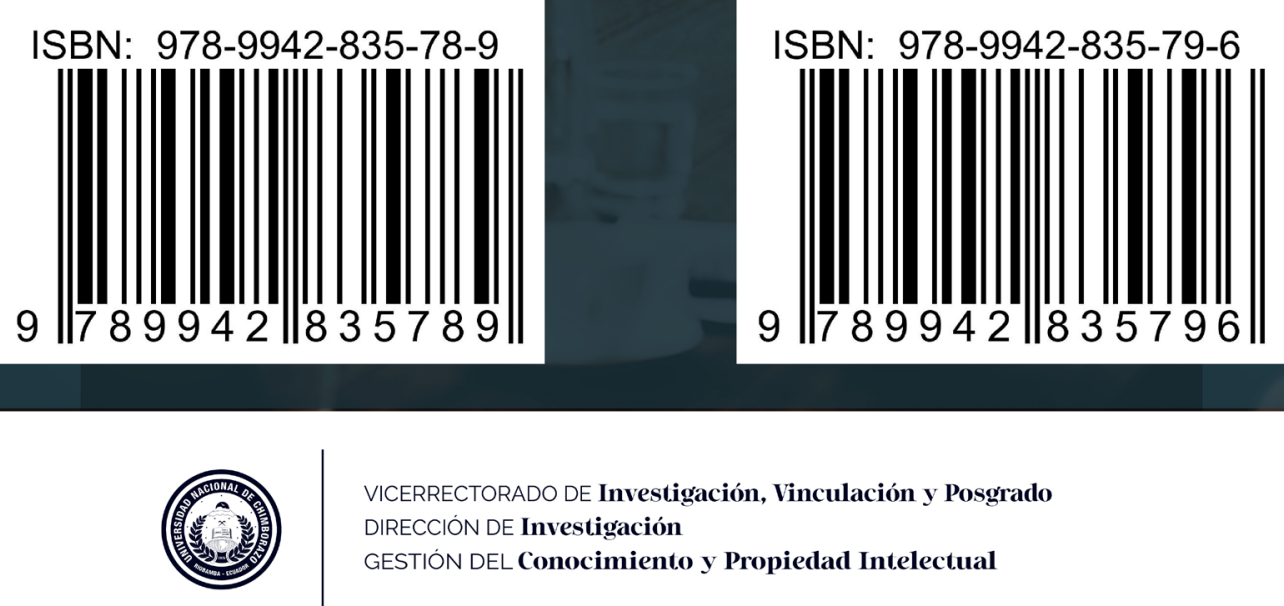

VICERRECTORADO DE Investigación, Vinculación y Posgrado DIRECCIÓN DE Investigación

GESTIÓN DEL Conocimiento y Propiedad Intelectual 